$\left(3,3^{\prime \prime}\right)$-Linked Biflavanones from Ouratea spectabilis and Their Effect on the Release of Pro-inflammatory Cytokines by THP-1 Cells

Marina P. Rocha,${ }^{\dagger}$ Priscilla R. V. Campana,${ }^{\dagger}$ Rodrigo M. Pádua,${ }^{\dagger}$ José D. S. Filho,${ }^{\star}$ Daneel Ferreira, ${ }^{\S}$ and Fernão C. Braga ${ }^{\dagger, *}$

\title{
SUPPLEMENTAR INFORMATION
}

NMR data of isolated compounds:

BIF1 - ouratein A (1)

BIF 2 - ouratein B (2)

BIF 3 - ouratein C (3)

BIF 4 - ouratein D (4)

HRESIMS data of ourateins A-D (1-4) 
S9E・

TLE $\varepsilon^{\circ}$

Ђ $8 \varepsilon^{\circ} \nabla$

$06 \varepsilon^{\circ} \nabla$

$887^{\circ} 9$

$767^{\circ} 9$

$\angle O S^{\circ} 9$

DIS. $S^{\circ}$

$288^{\circ} 9$

$888^{\circ} 9$

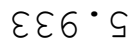

$8 \varepsilon 6^{\circ} 9$

ง 9 9

$\angle \angle 9^{\circ} 9$

$\varepsilon G I \cdot L-$

$S L I \cdot L-$

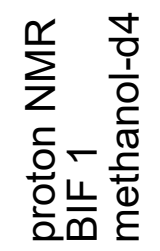

ڤू

$\stackrel{n}{\circ}$

ㅇ.

म

ㅁ.

เก

ㅇ.

6

$\stackrel{?}{\circ}$

6 
ง $9 \cdot 9$

$\angle \angle 9^{\circ} 9$

$\varepsilon S I \cdot L-$ $S L I \cdot L$ 
$887^{\circ} 9-$

$767^{\circ} \cdot 9$

$\angle O S^{\circ} S$

DIG. $S^{\circ}$

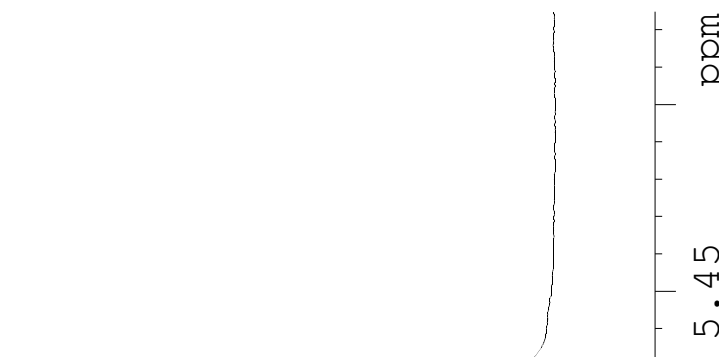

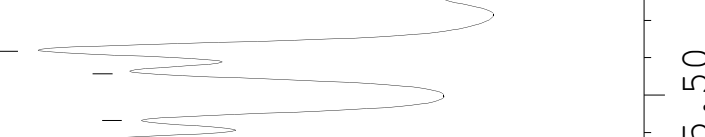

เก

$n$
-5

เก

8

เก

0
-6

เก

$\stackrel{\circ}{r}$

ம

$\stackrel{n}{\sim}$

$\dot{\circ}$

$\circ$
$-\infty$

ம்

\llcorner
$\infty$
$\infty$

$288^{\circ} 9$

$888^{\circ} 9$

$\varepsilon \varepsilon \sigma^{\circ} \varsigma^{-}$

$8 \varepsilon 6^{\circ} 9^{-}$ 
ธ9ย・

ILE' $\bar{D}$

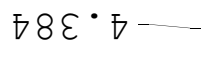
$06 \varepsilon^{\circ} \hbar-$

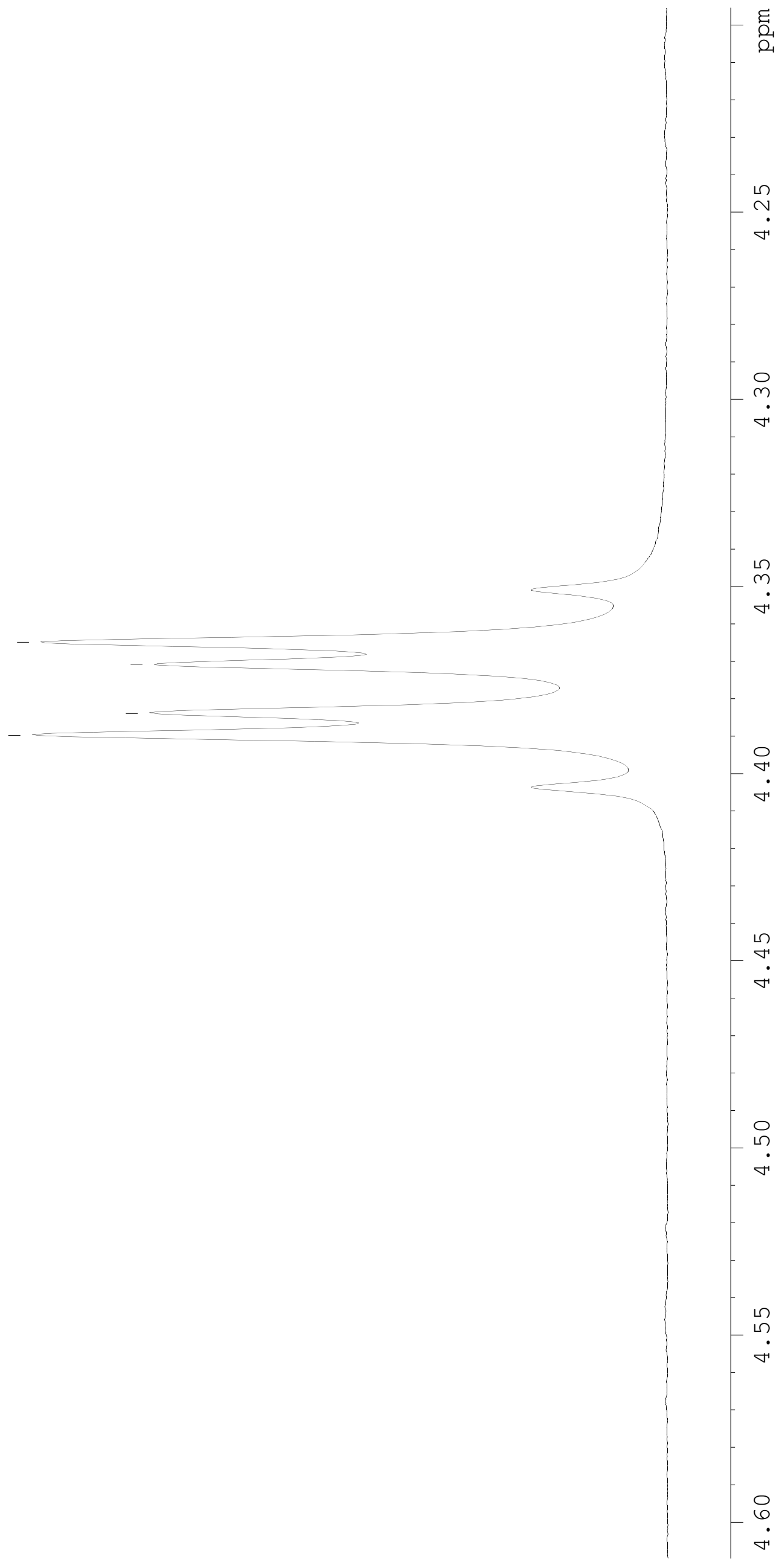




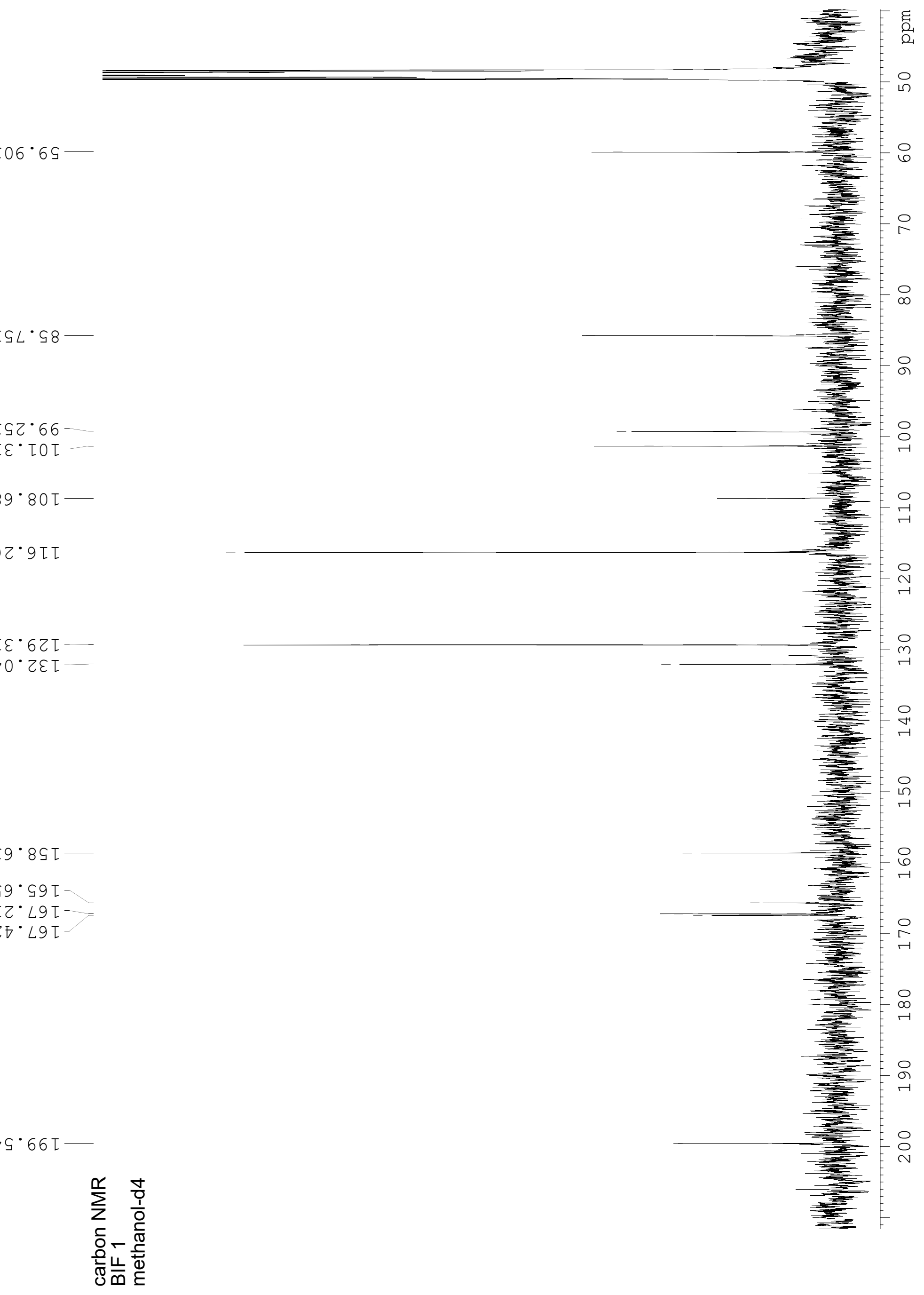



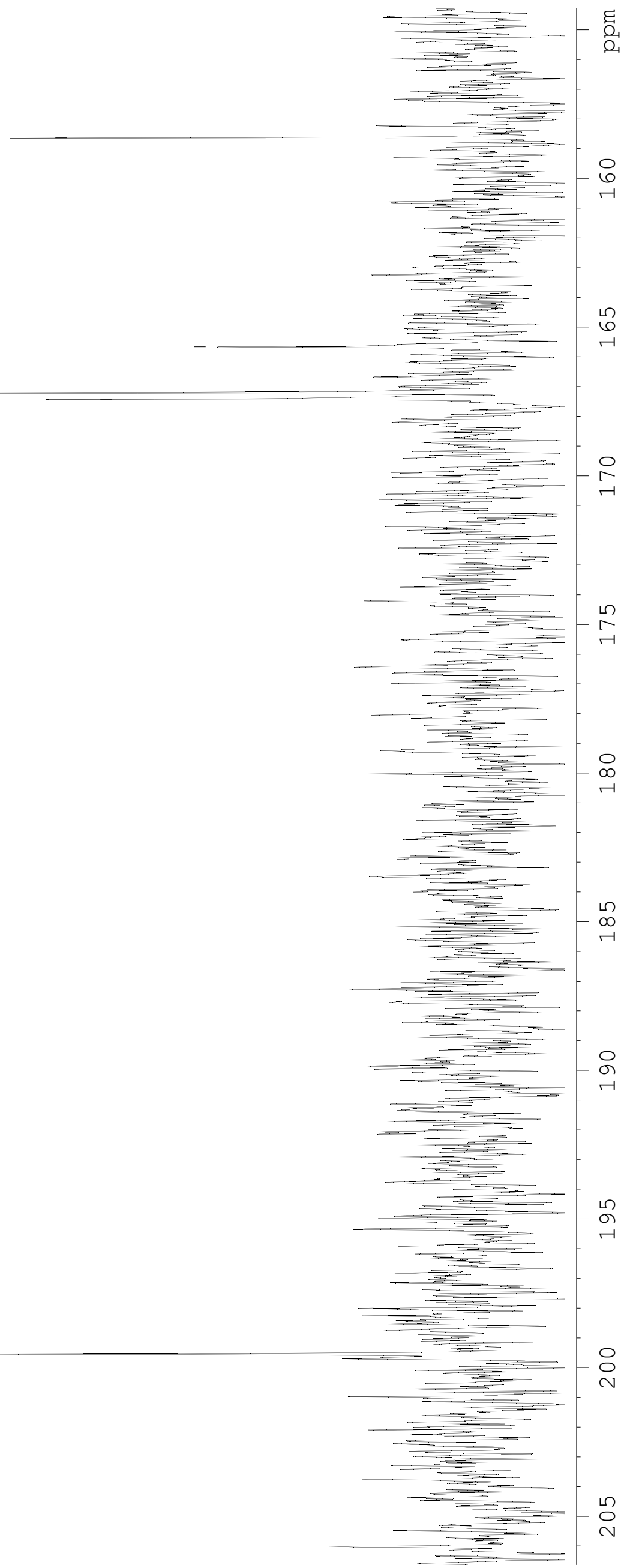
$\angle 89 \cdot 80 I$

$09 \tau \cdot 9 \tau I-$

$8 \tau \varepsilon \cdot 6 \tau \tau-$

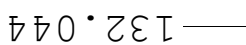

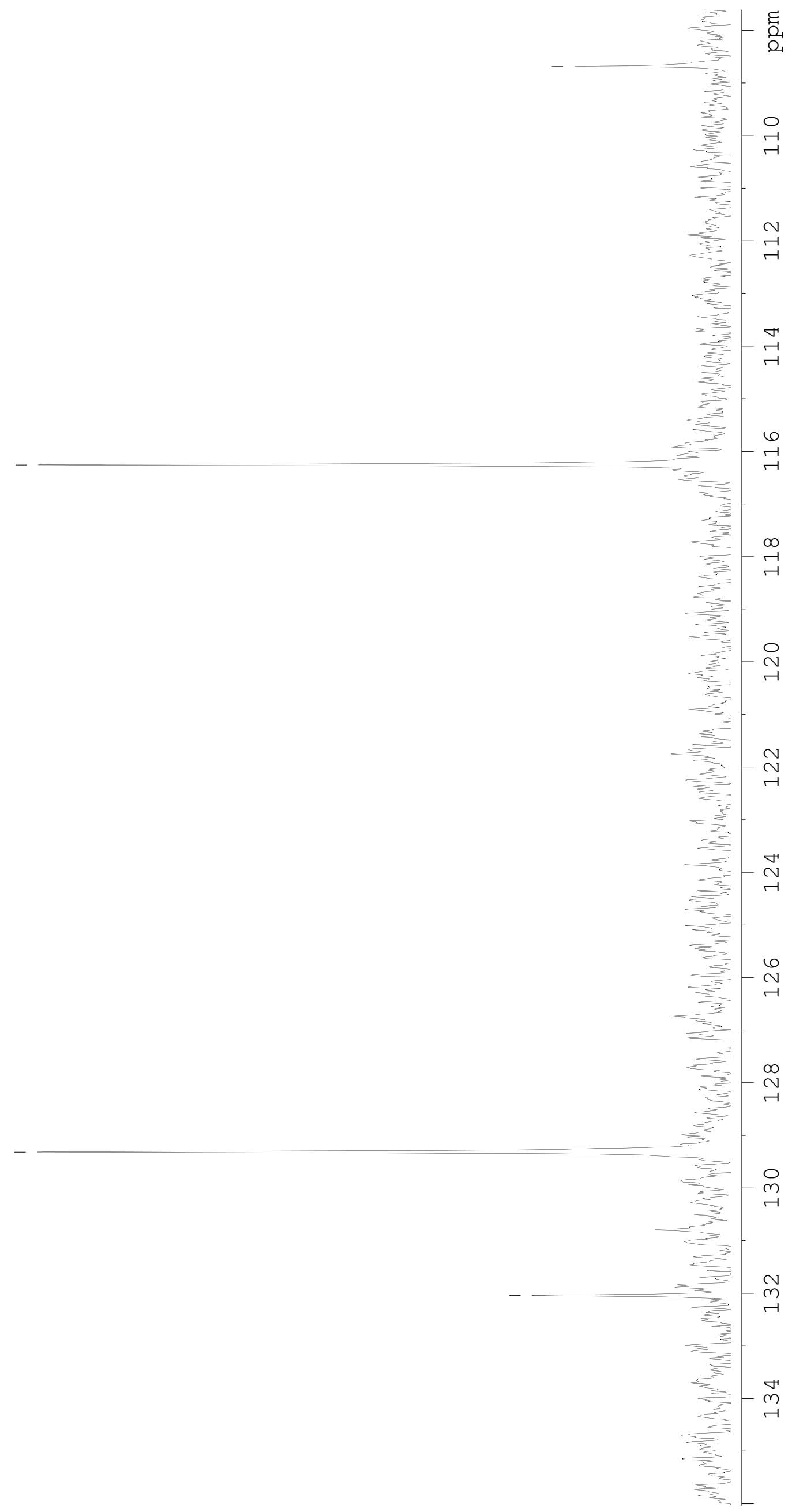


ISL・SB

$\varepsilon \varsigma 乙 \cdot 66$

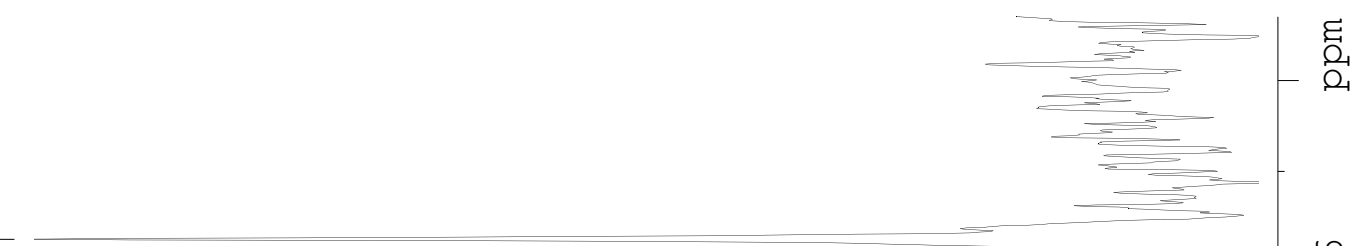

$\square$

$\longrightarrow$

$\underline{\underline{E}}$

$=$

$\ldots$

$\underline{=}$

$=-$

$=$

$\ldots$

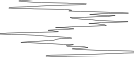

$=$

$-$

$=$

$=$

$=$

$=$

$=$

$\ldots$

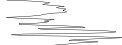

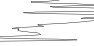

$=$

$=$

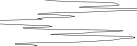

$+$

$+$

$+\underline{2}$

I

$\angle 89^{\circ} 80 T$

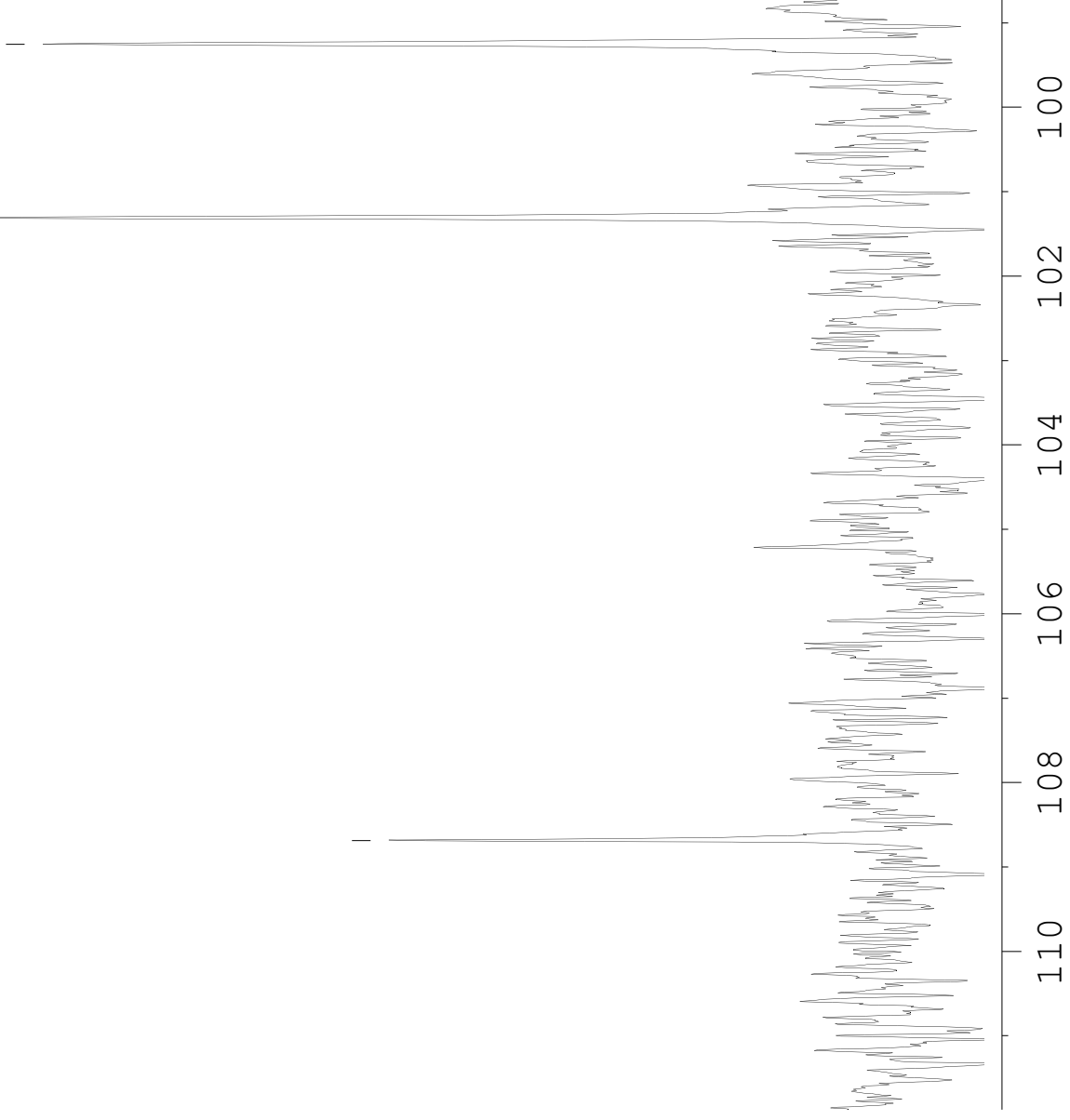




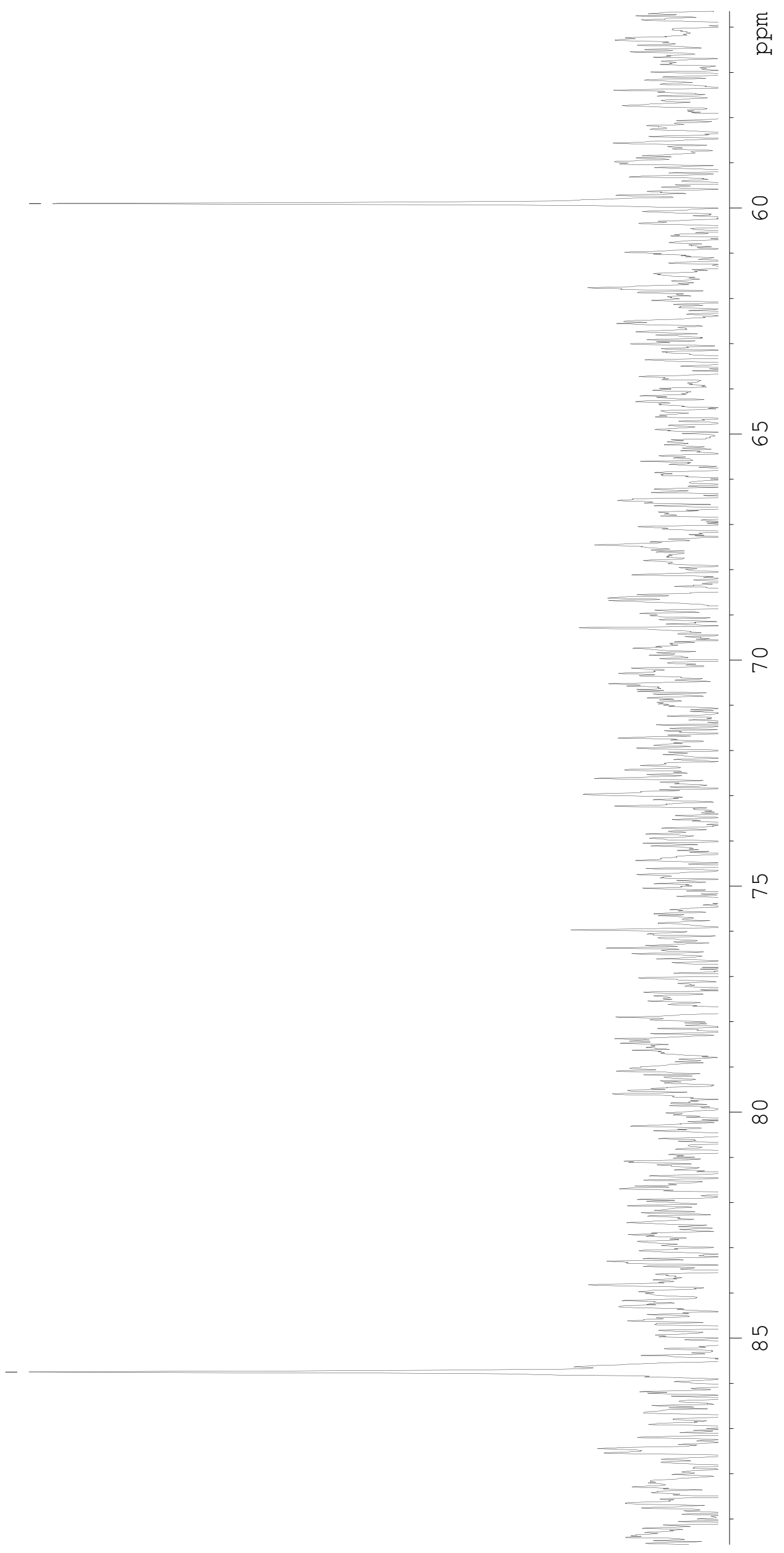

โSL・S৪ - 
IZS.89

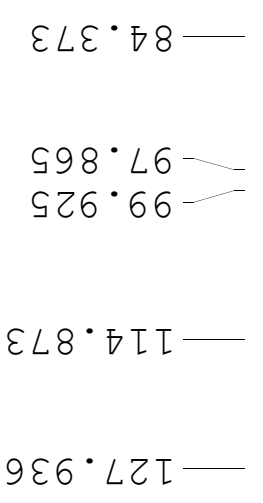

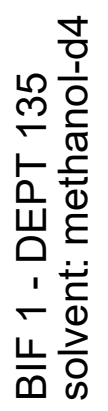

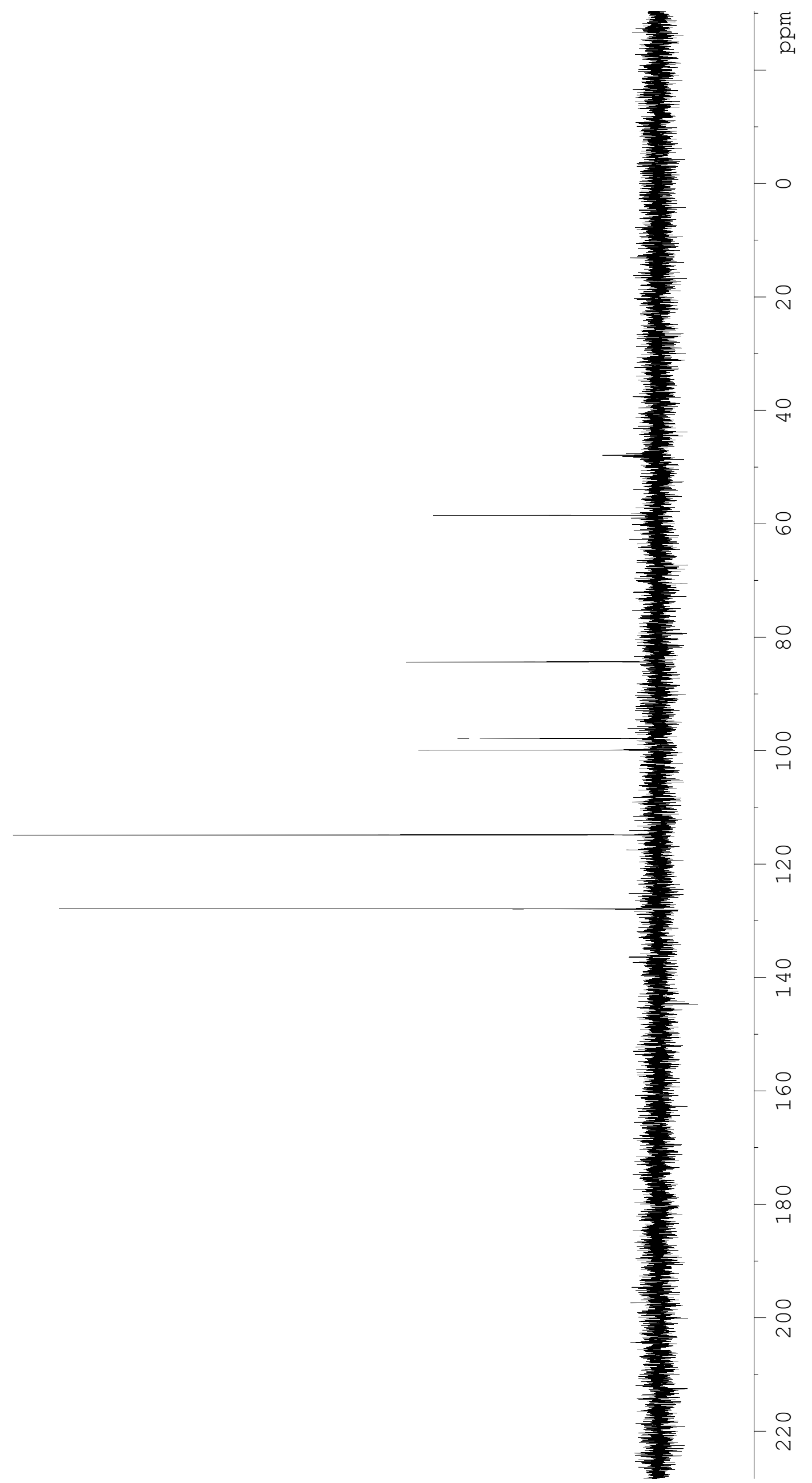


$998^{\circ} \mathrm{L6}$

$926 \cdot 66$

$\varepsilon \angle 8^{\circ}$ †I

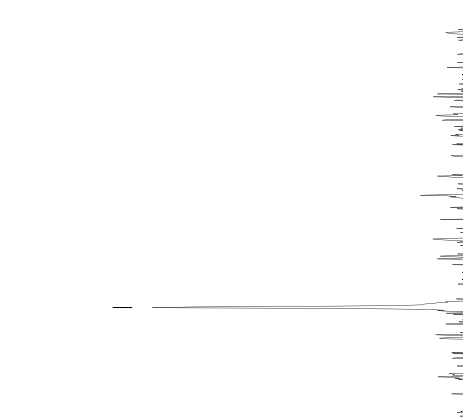

हू 


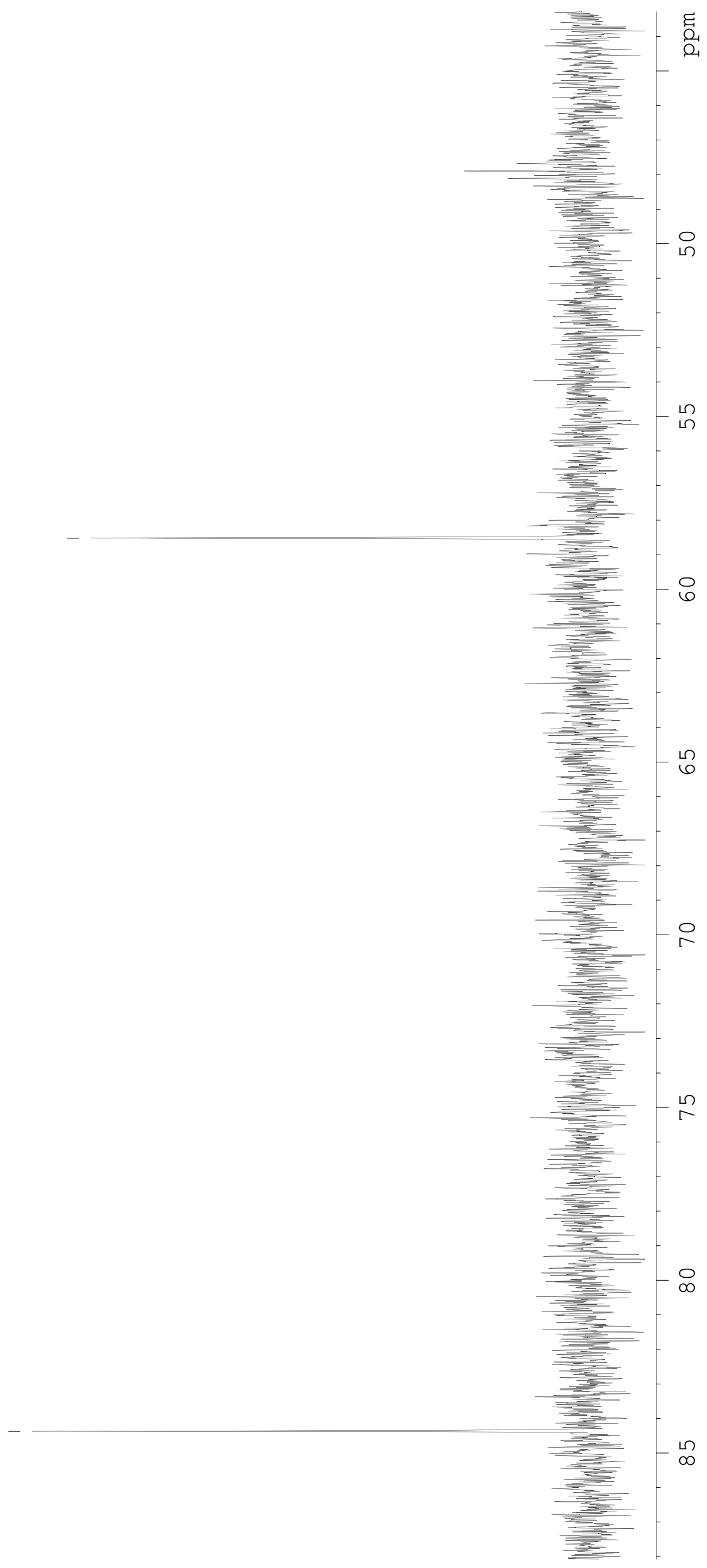




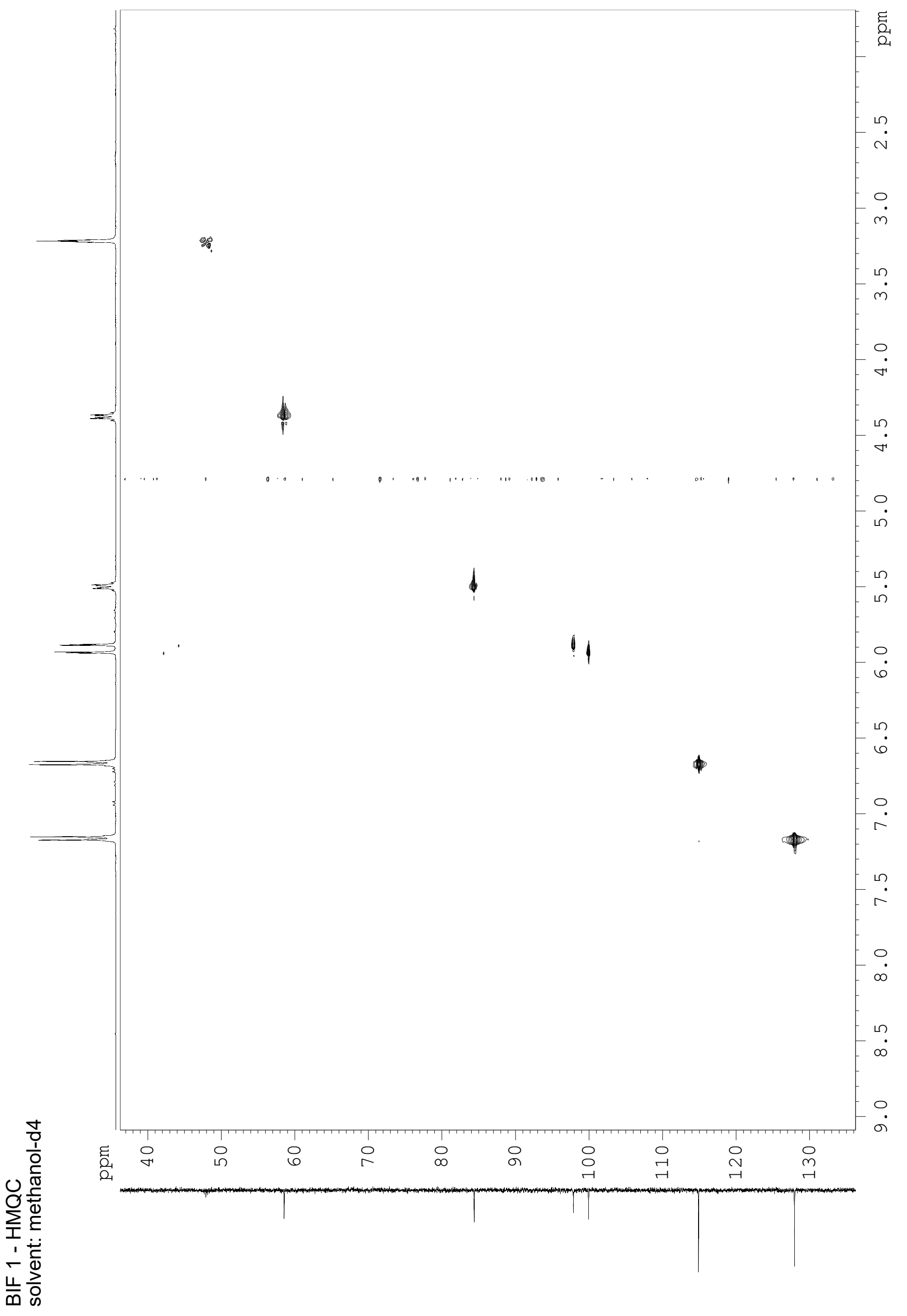




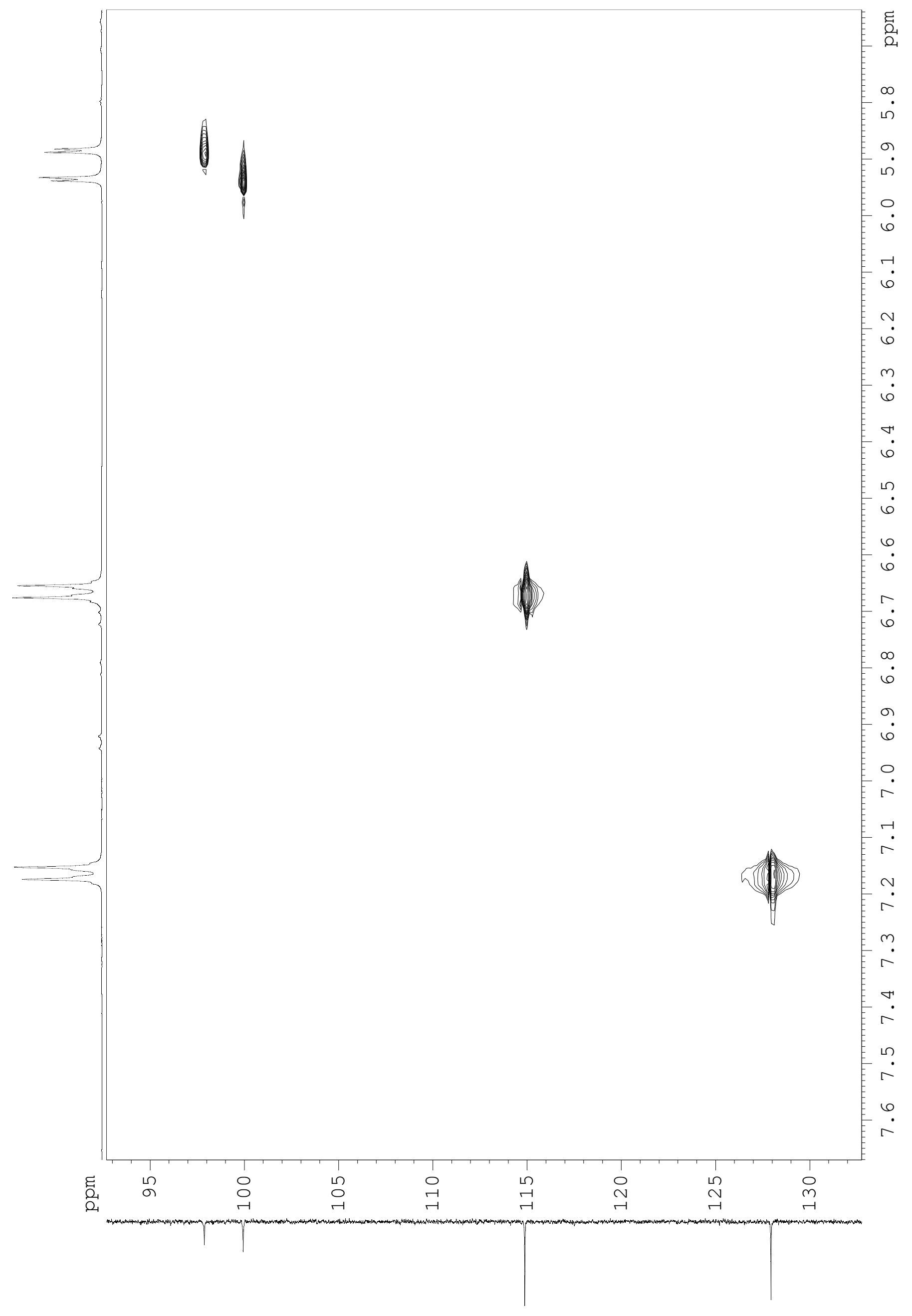




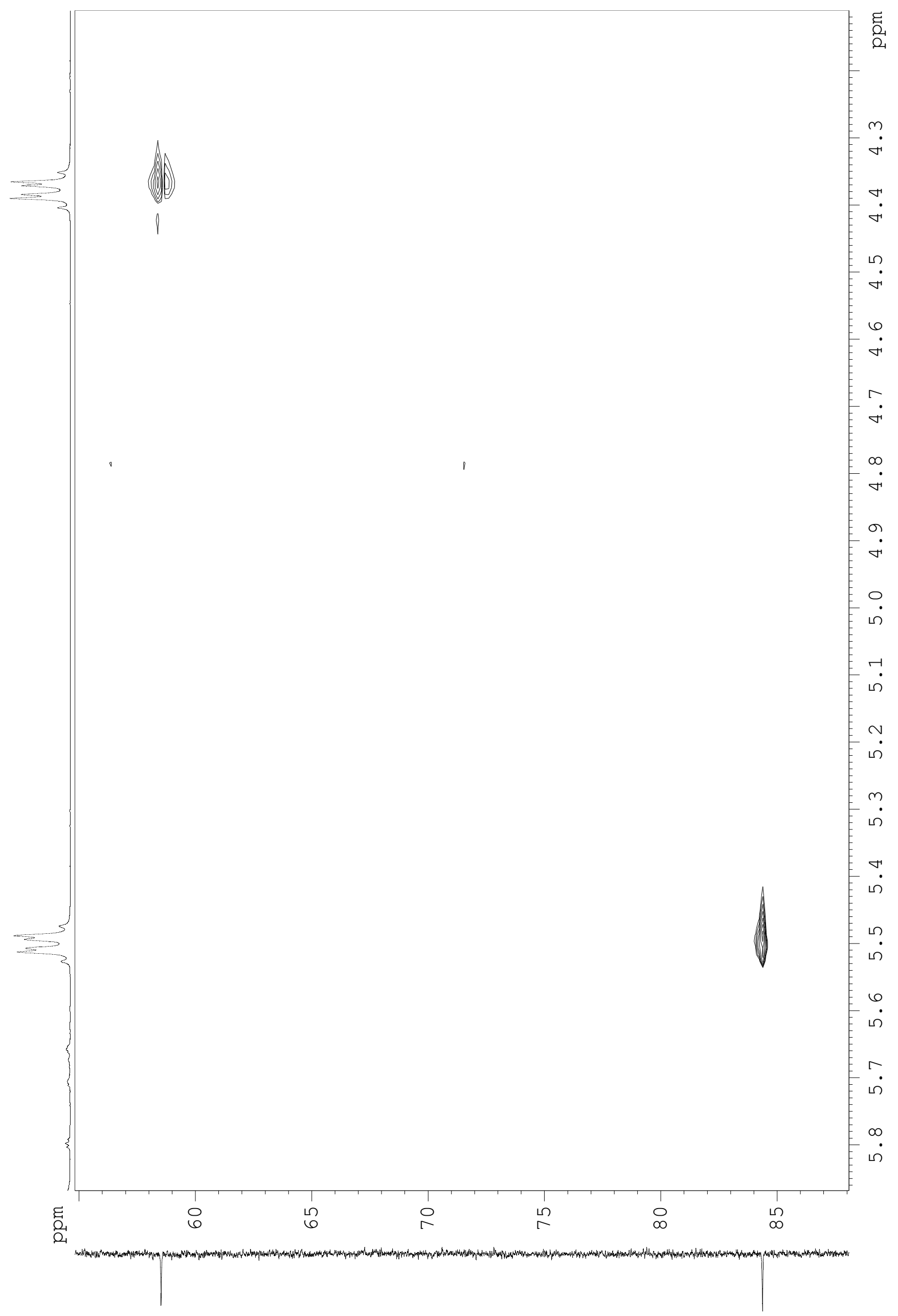




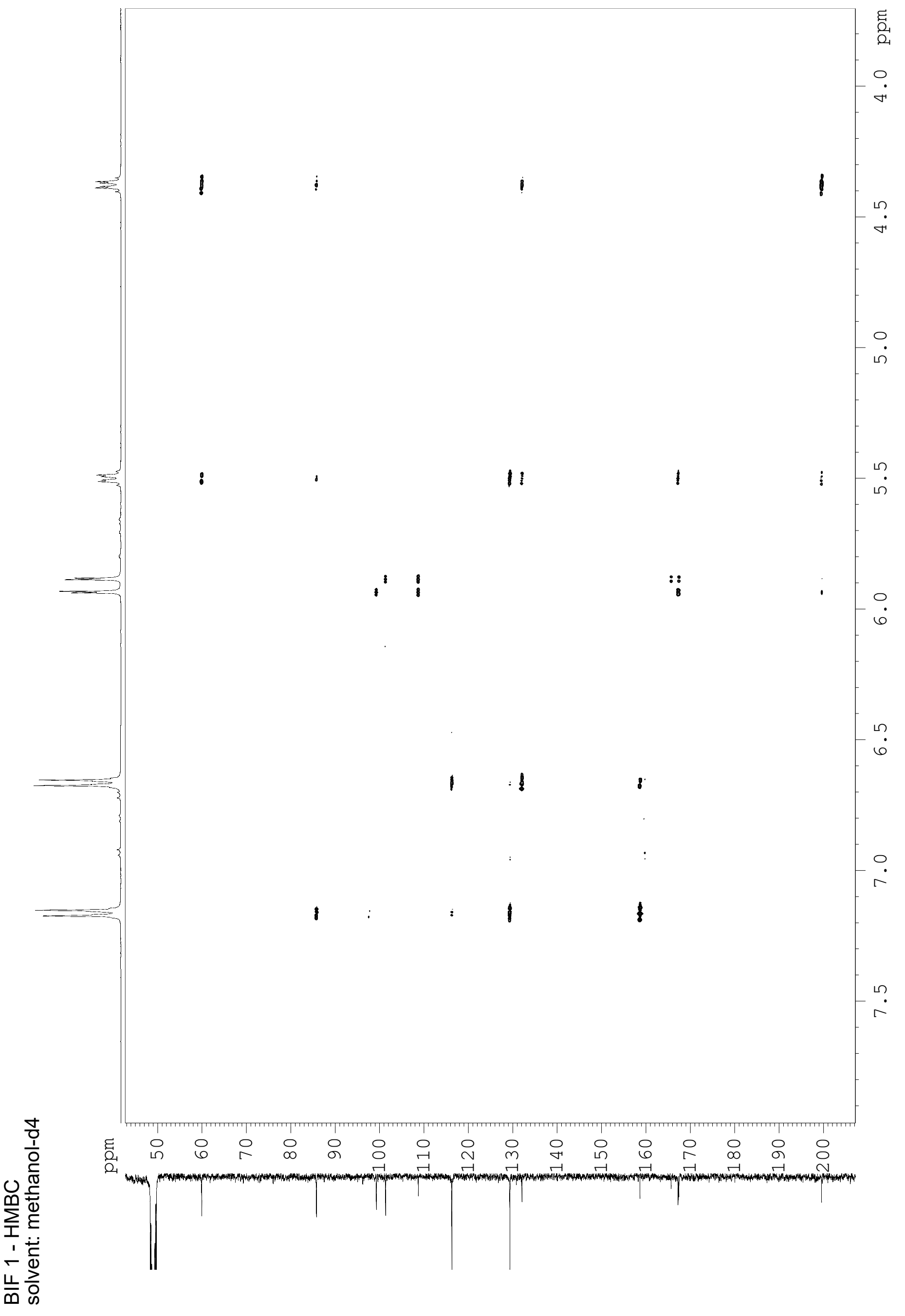




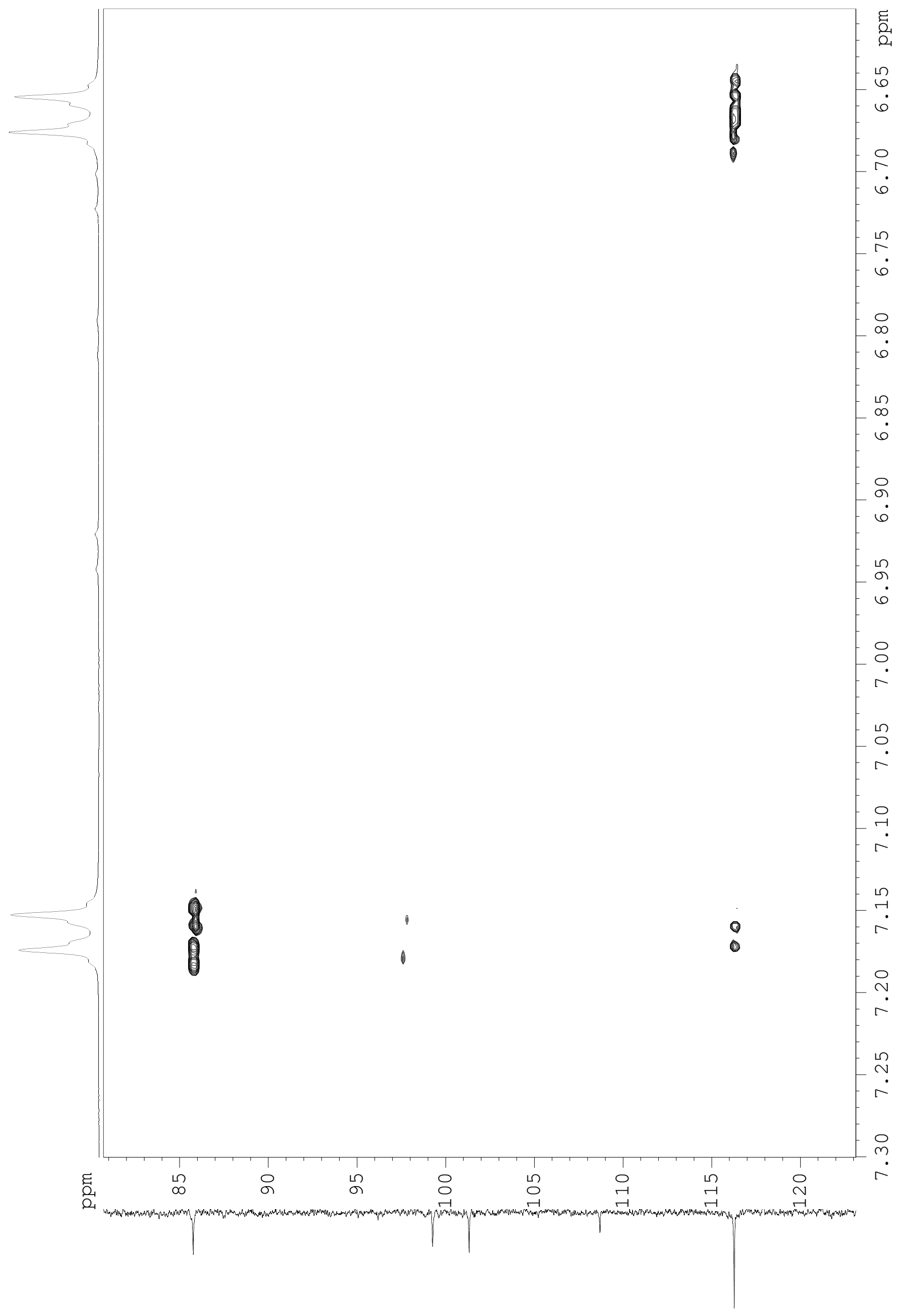




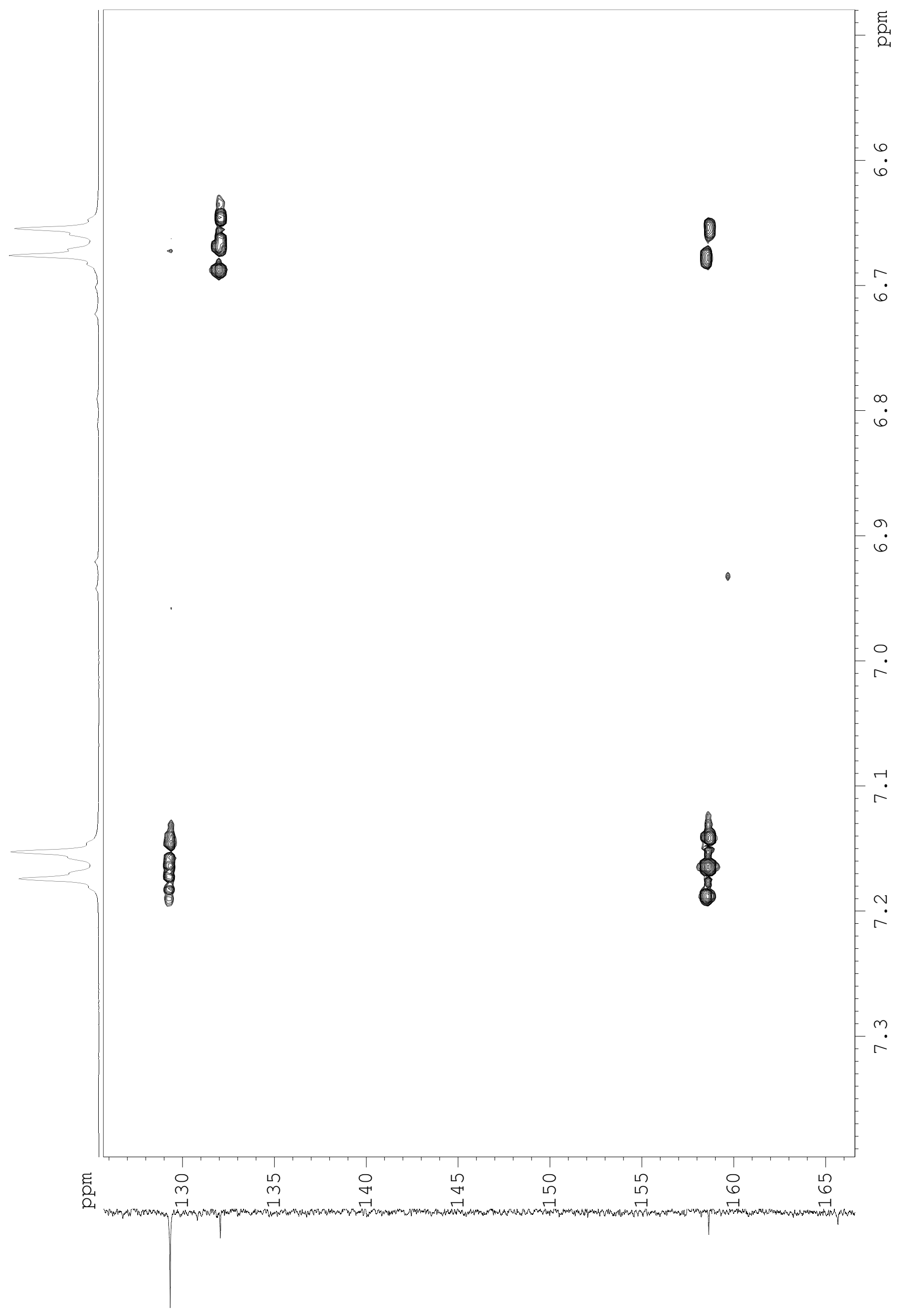




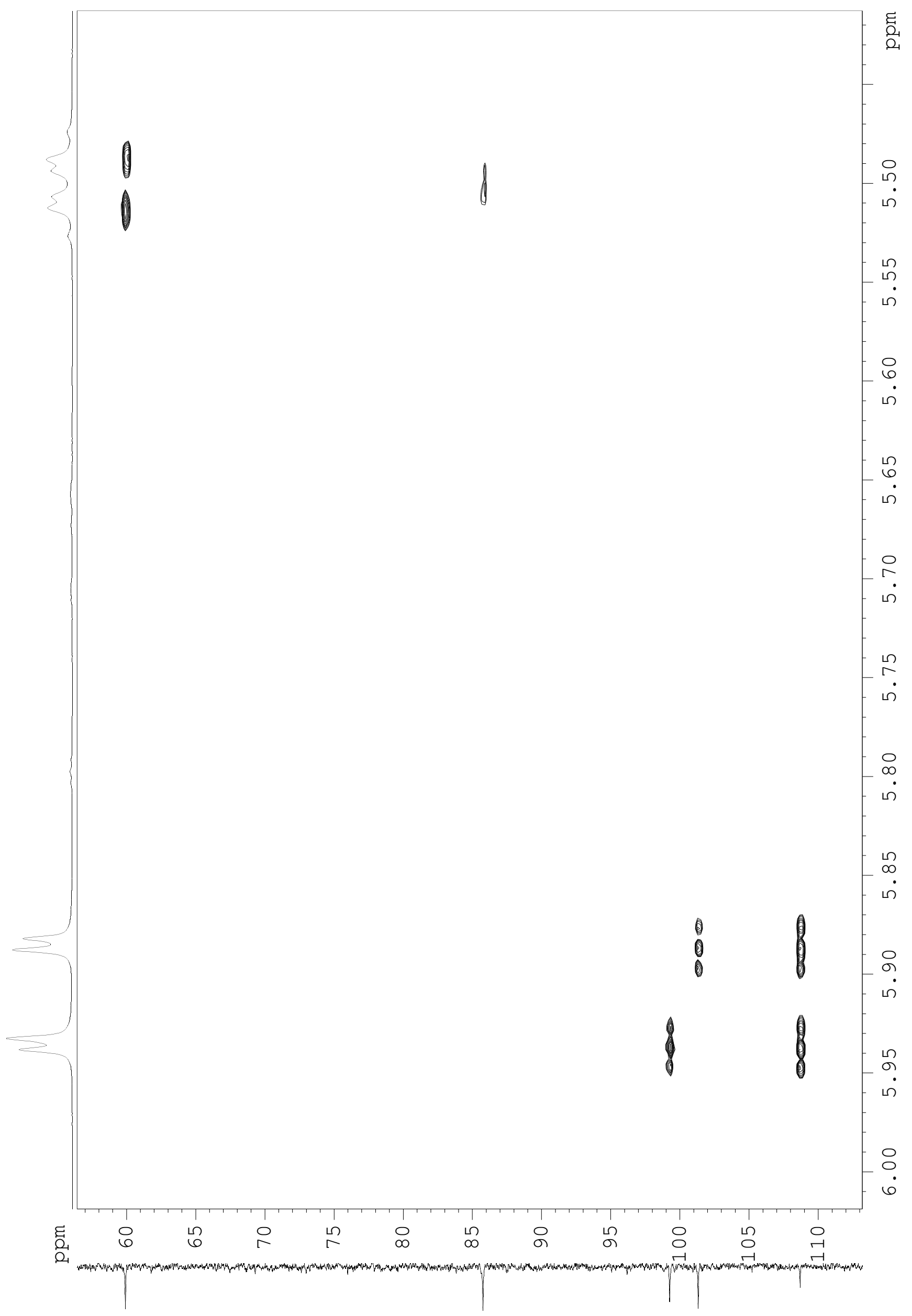




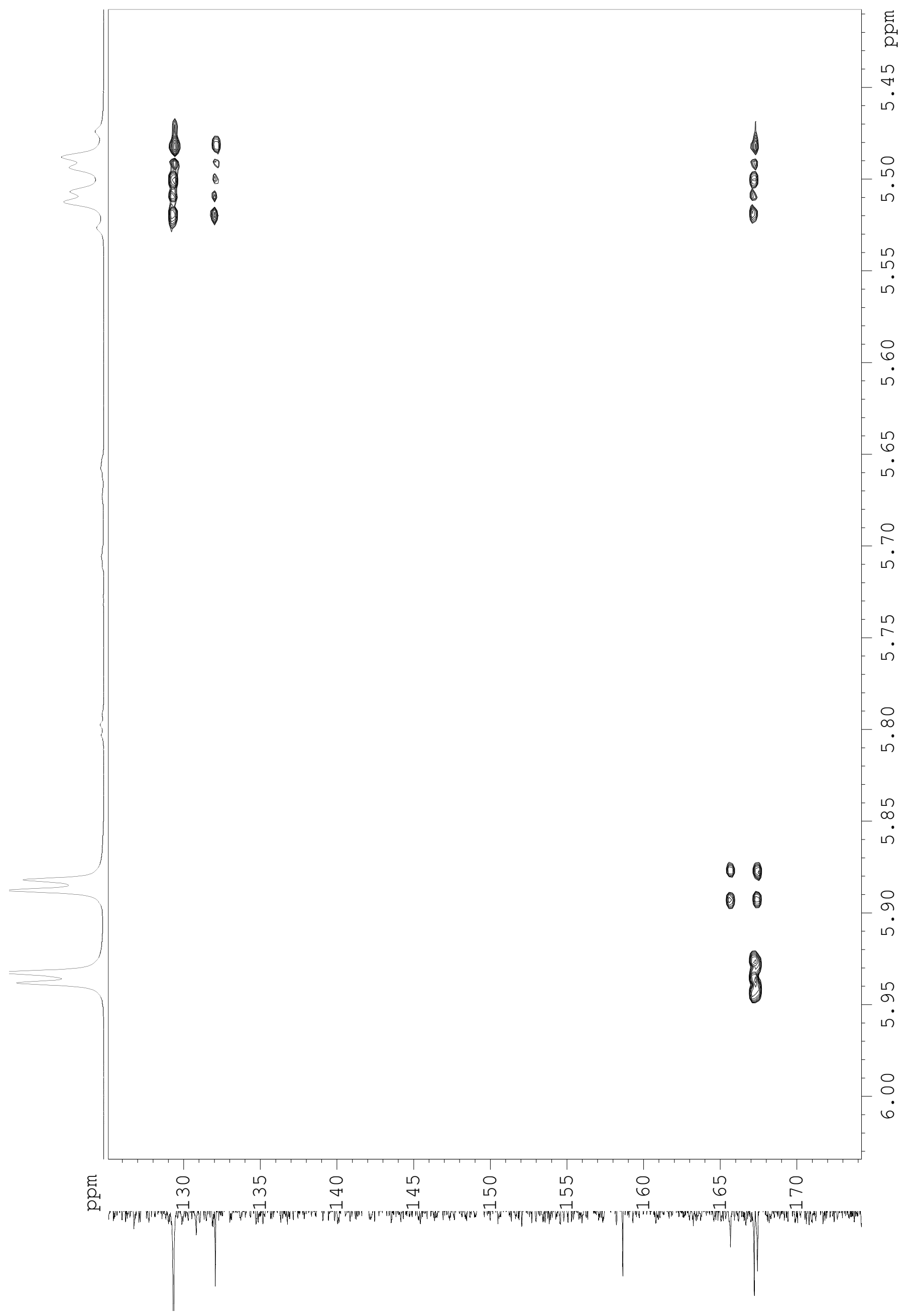




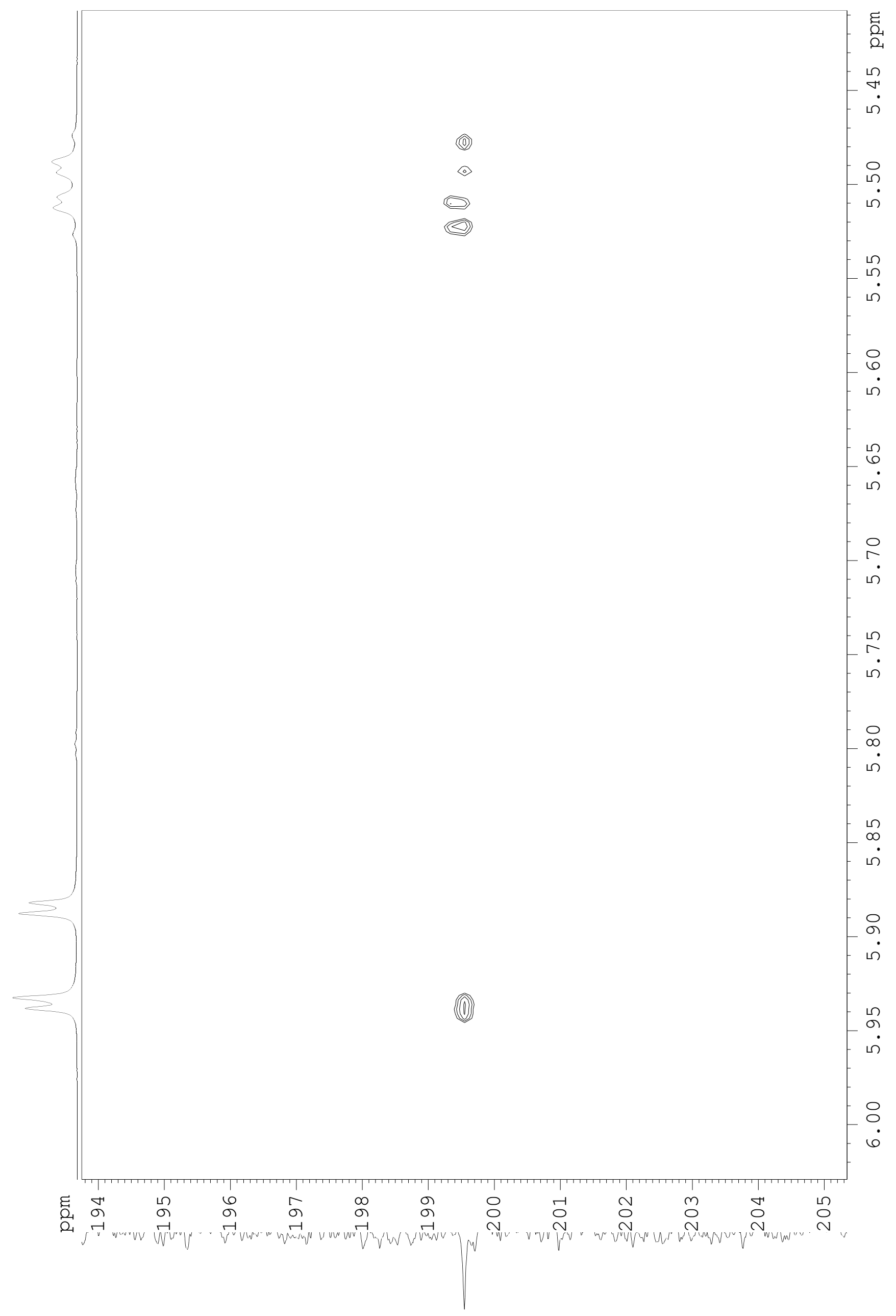




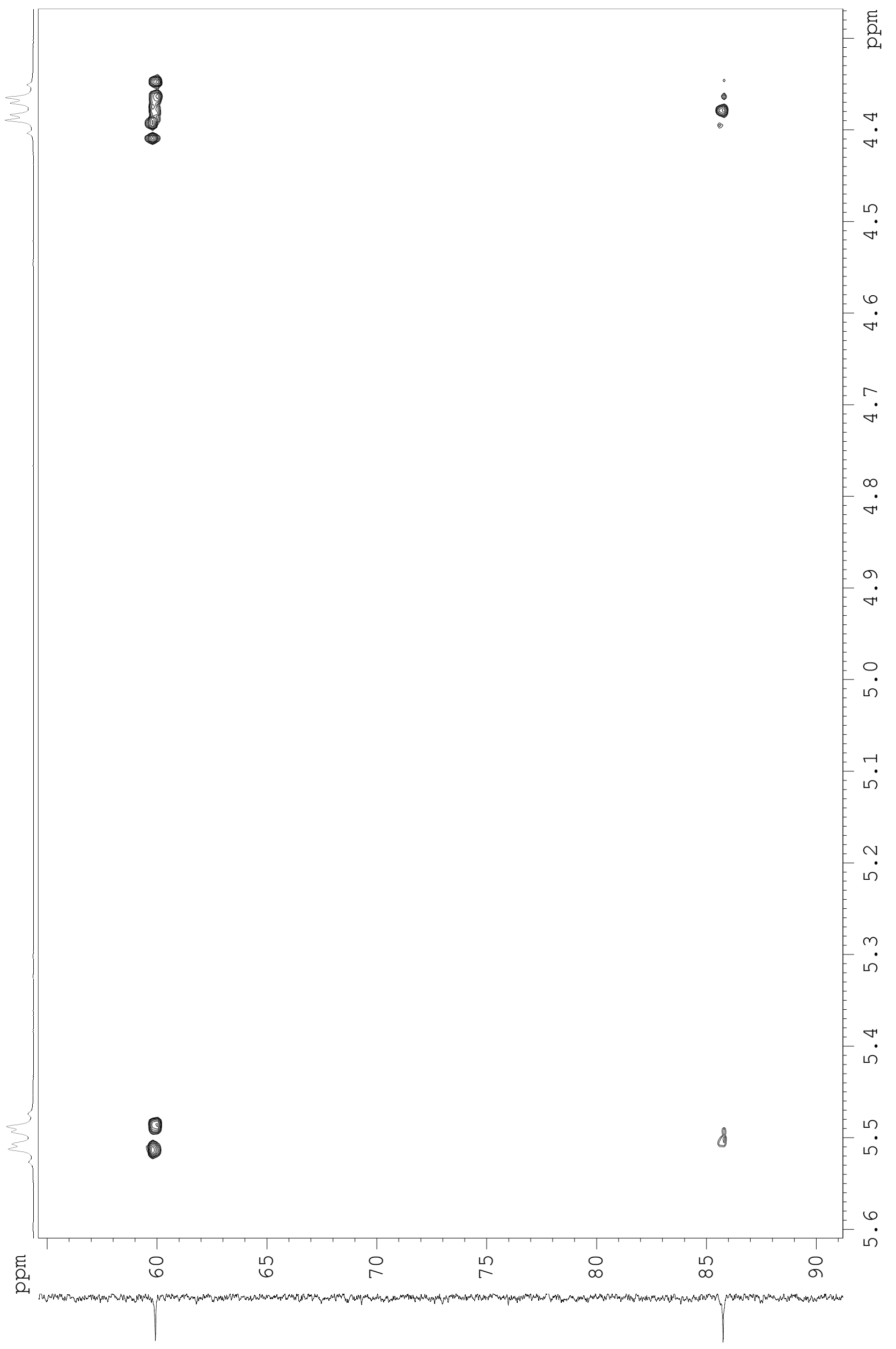




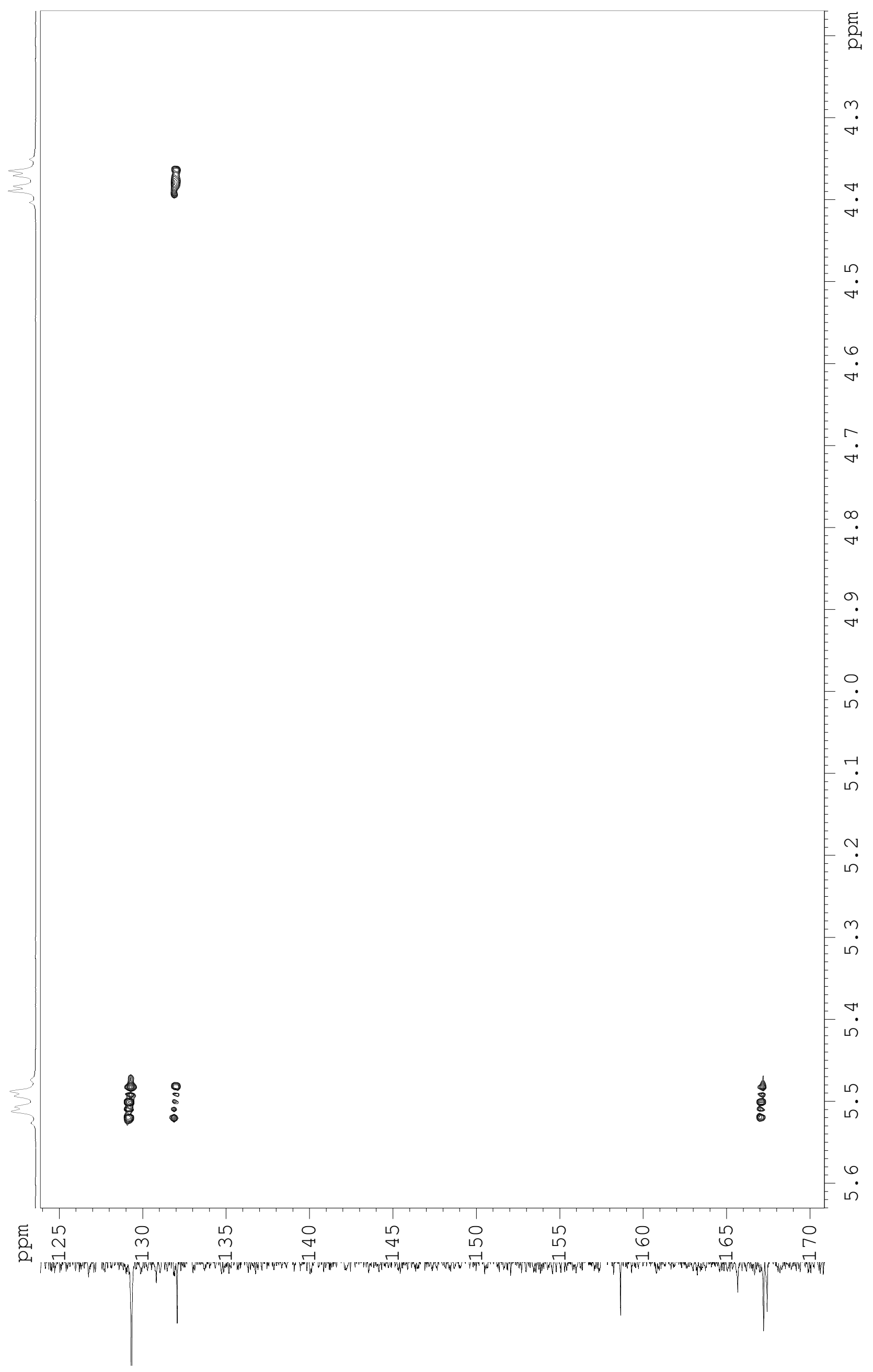




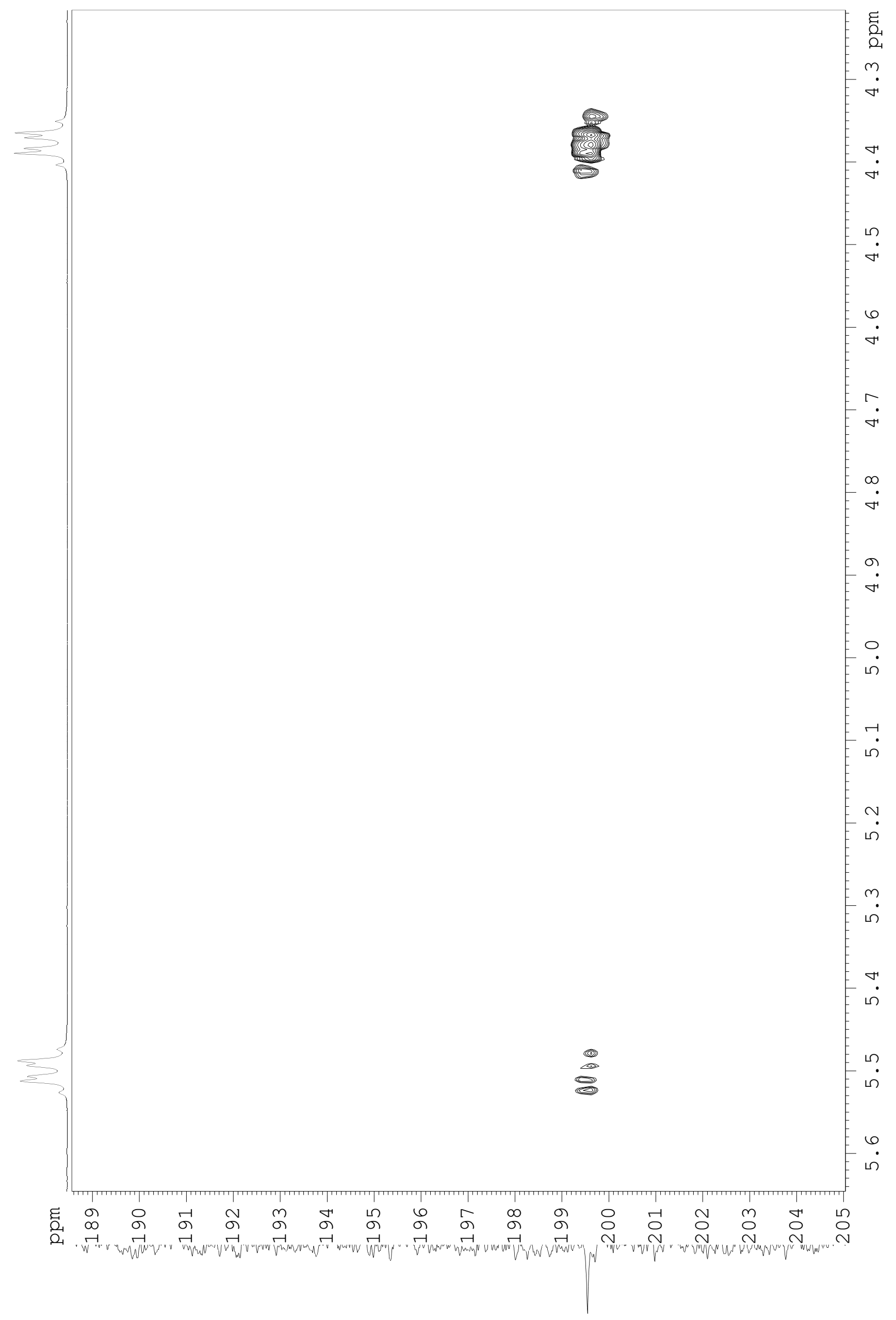


$090^{\circ} \mathrm{I}-$
$8 \mathrm{LO} 0^{\circ} \mathrm{T}-\mathrm{\gamma}$ $960^{\circ} \mathrm{T}-$ $\varepsilon 8 \tau^{\circ} \mathrm{I}$ $6 \overline{\sigma^{\circ}} \cdot \tau$.

Zโ $8^{\circ} \tau-$ $\angle \varepsilon 8^{\circ}$ $\mathrm{L} 68^{\circ} \mathrm{T}$ $290^{\circ} \mathrm{Z}$ $260^{\circ} \mathrm{Z}-$ $602 \cdot 2$

$\angle O Z^{\circ} \varepsilon$

โน乙

sโz

$0 S{ }^{\circ} \varepsilon$

$667^{\circ} \varepsilon$

$9 \tau 9^{\circ} \varepsilon$

$8 \varepsilon 9^{\circ} \varepsilon$

$699^{\circ} \varepsilon$

$\angle 8$

$90 \sigma^{\circ}$
SIt

โZஏ.

OEF

$8 \nabla \sigma \cdot \sigma$

ஏ乙L・

$\varepsilon 87^{\circ} 9$

דOS.s

$929^{\circ} 9$

$\angle 98^{\circ} 9$

ZL8.

$\angle\left[6^{\circ} G^{\circ}\right.$

$\varepsilon 26^{\circ} 9$

$[20 \cdot 9]$

$429 \cdot 9$

TS9.9

$\varepsilon \angle 9 \cdot 9$

$076 \cdot 9$.

$6 \varepsilon \tau \cdot L$

$09 I^{\circ}$

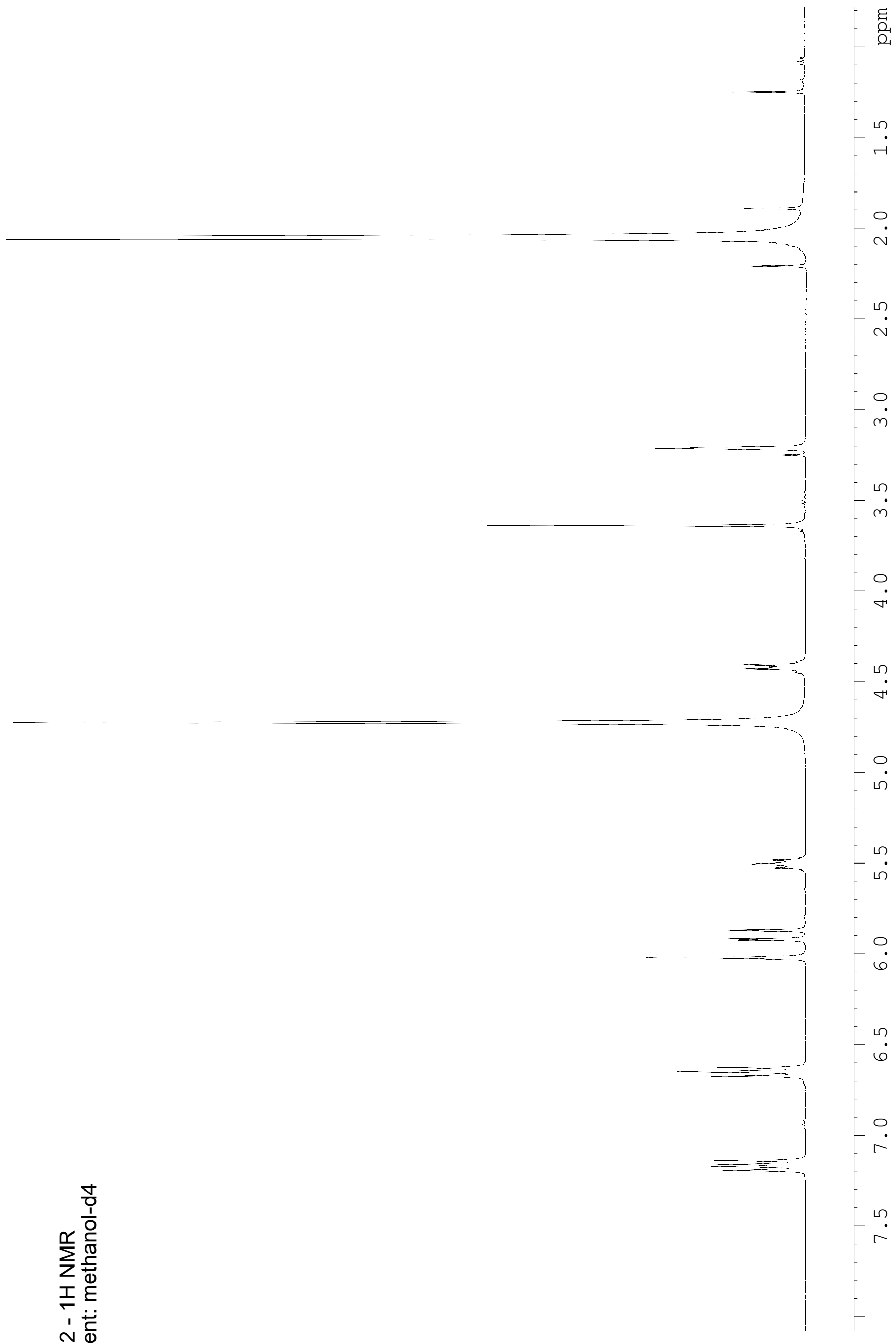

$\varepsilon 6 I^{\circ}$

픙 
$498^{\circ} 9$

$2 \angle 8^{\circ} \cdot-$

$L\left[6^{\circ} \cdot G\right.$

$\varepsilon 26^{\circ} 9-$

โ20 9

$429 \cdot 9$

IS9.9

$\varepsilon \angle 9 \cdot 9$ -

$076 \cdot 9$

$6 \varepsilon I \cdot L-$

$09 I \cdot L-$

ZLI $L$

$\varepsilon 6 \tau \cdot L$

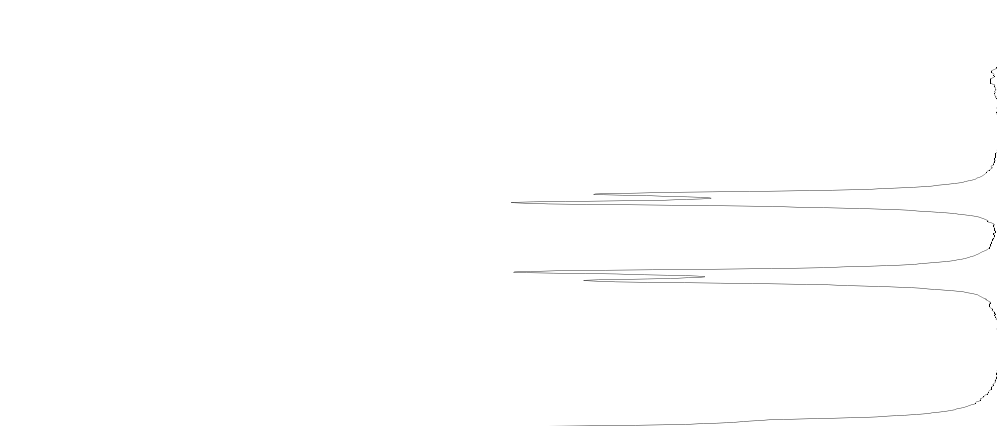


$\angle 8 \varepsilon^{\circ} t$

$90 \sigma^{\circ}$

SI万.

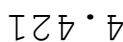

OE币・

8も币・元

ஏてL・元

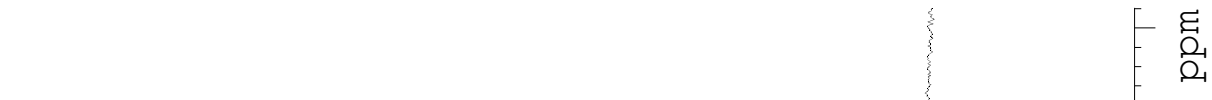

$\stackrel{4}{+}$

$\stackrel{4}{\circ}$

6

$\dot{\gamma}$

r

$\infty$

$\dot{r}$

a

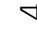

$\stackrel{0}{\dot{0}}$

$-\dot{r}$

$\stackrel{\sim}{\sim}$

m.

ウ

$\varepsilon 87^{\circ} \varsigma^{\circ}$

DOS. 9

9ZS. 9

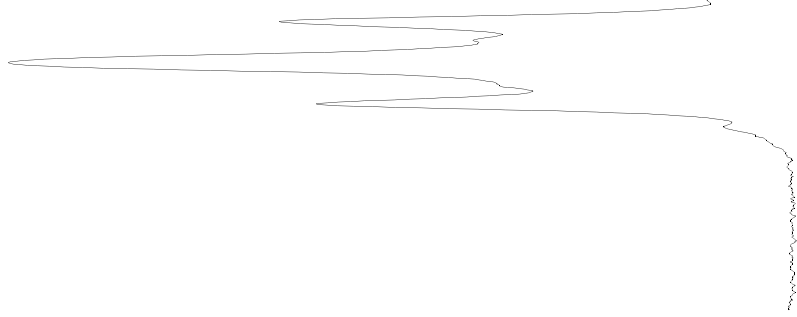

\llcorner

- ம

ம 
$\angle O Z \cdot \varepsilon-$

โโ乙

$\varsigma \tau 乙 \cdot \varepsilon-$

$0 \varsigma 乙 \cdot \varepsilon$

$66 \sigma^{\circ} \varepsilon$

$9 I G^{\circ} \varepsilon-$

$8 \varepsilon 9 \cdot \varepsilon-$

$699 \cdot \varepsilon$

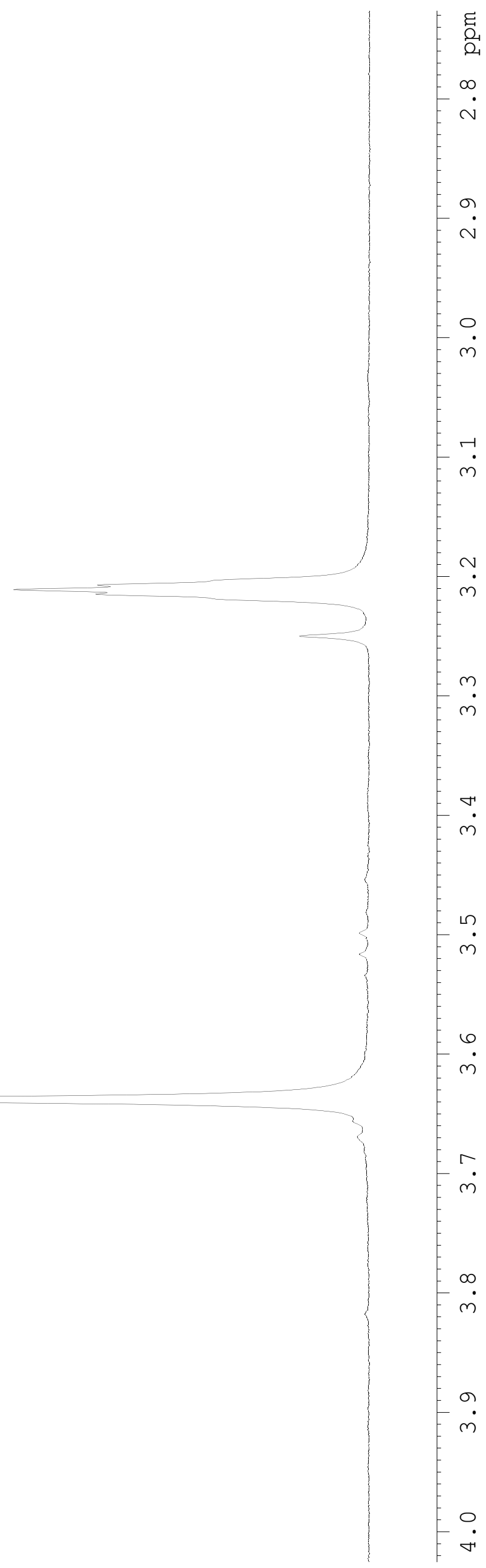




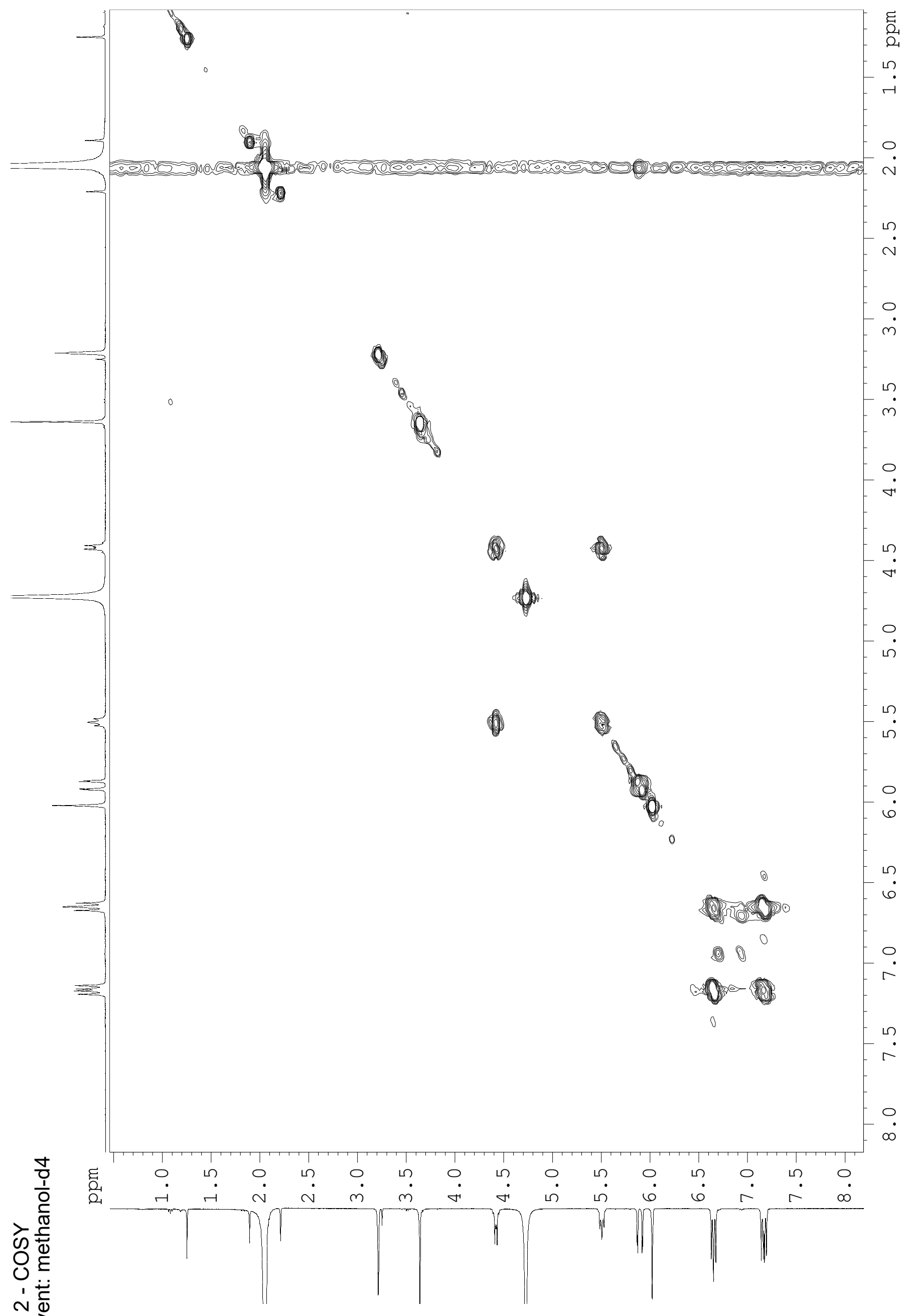

寅亭 


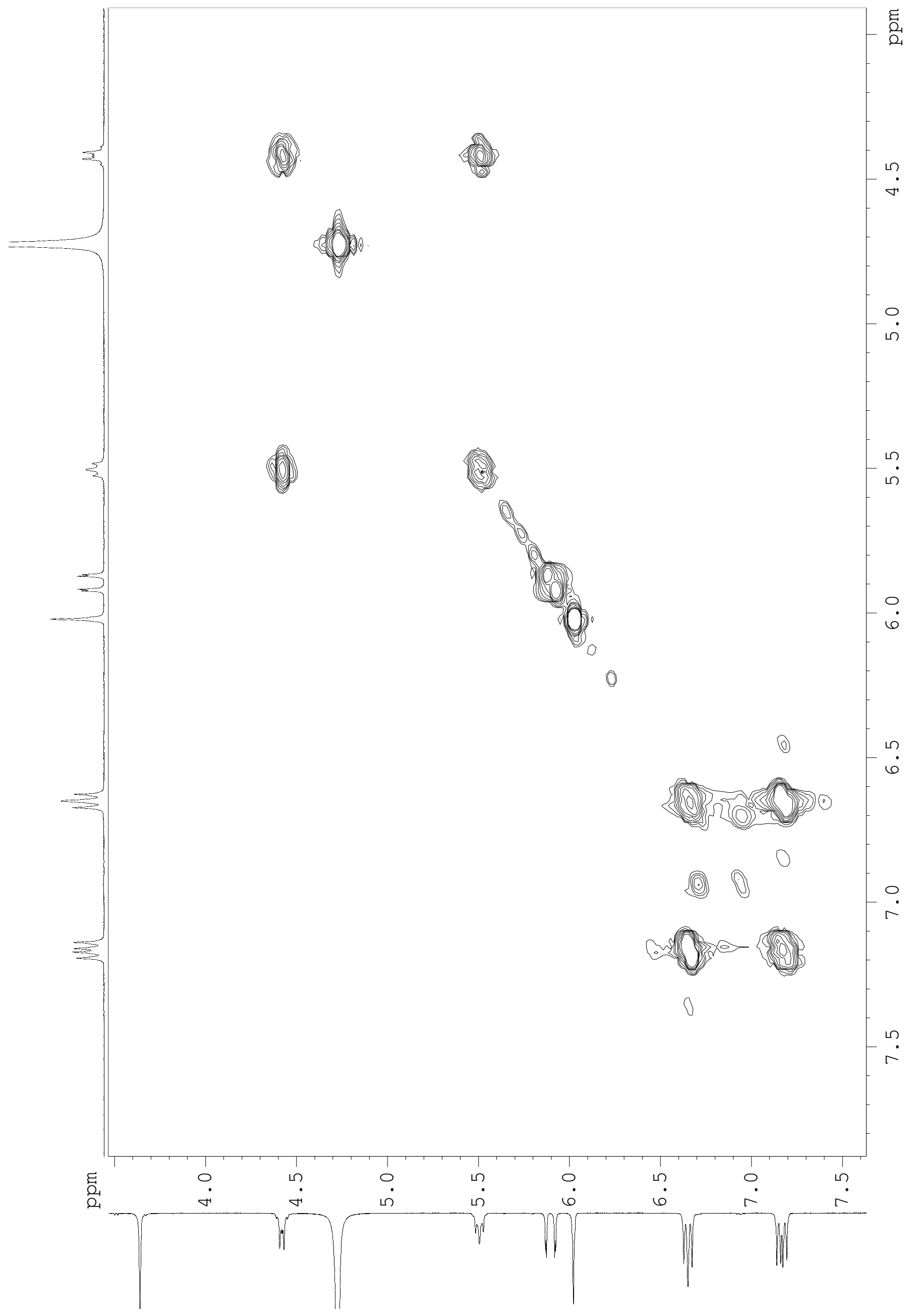


$0 \angle 6 \cdot 97$

$\angle \nabla Z \cdot 87$

$088^{\circ} \nabla 9$

$609^{\circ} 89$

$608^{\circ} 89$

ง0ゥ・ர8-

LLL・ $\nabla 8-$

o $\varepsilon \varepsilon \cdot 96-$

S7 $8 \cdot \angle 6$

I97.86

6 I6.66

โ8Z $\angle 0 I$

IE乙 80 T

ऽ $8^{\circ}$ †I

$968^{\circ}$ 万I I

$09 I \cdot G I T$

$9 \tau 0 \cdot 8 Z \tau$ -

$\angle 70 \cdot 8 Z \mathrm{I}$

oTS.0ET

Oெ $S^{\circ} 0 \varepsilon \mathrm{I}$

$S G E \cdot \angle S I$

$\angle O F \cdot \angle S T$

乙โ0.

$762 \cdot \nabla 9 I$

$\varepsilon 29 \cdot 99 \mathrm{I}$

$678 \cdot 99 \mathrm{I}$

$880 \cdot 99 \mathrm{I}$

$8 \varepsilon 9 \cdot 99 \mathrm{I}$

IZ0 86 I -

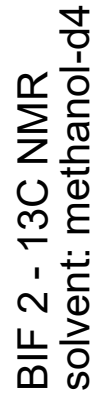

$278 \cdot 86 I$

$6 \varepsilon 9 \cdot 80 z$ 
IZ0・86 I

乙ち8・86I

$6 \varepsilon 9 \cdot 80 乙$ 
$S \subseteq \mathcal{E}^{\circ} \angle S I-$

$\angle O D^{\circ} \angle S I$

乙โ0・

$762 \cdot 79 \tau$

$\varepsilon 29 \cdot 99 \mathrm{~L}$

$678 \cdot 99 \mathrm{~L}$

$880 \cdot 99$ I

$8 \varepsilon 9 \cdot 99 I-$

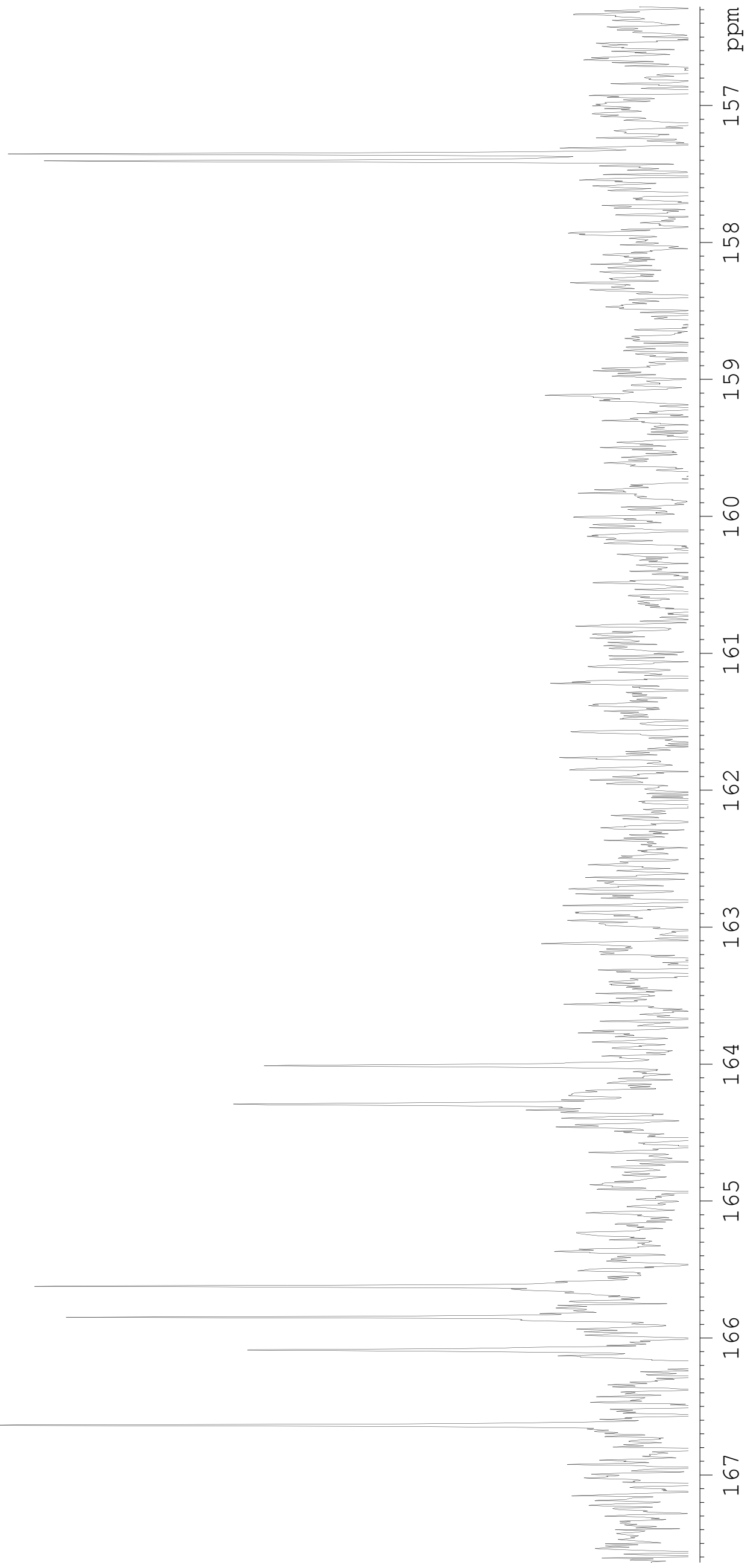


$\left[8 \tau^{\circ} \angle O L\right.$

โE乙・80

S $8^{\circ}$ 万I

$968^{\circ}$ 万I

$09 I \cdot G I T$

$9 \tau 0 \cdot 8 Z \mathrm{~T}$

$\angle \nabla 0 \cdot 8 Z T$

OLS.OEL-

Oெ $S^{\circ} 0 \varepsilon L$

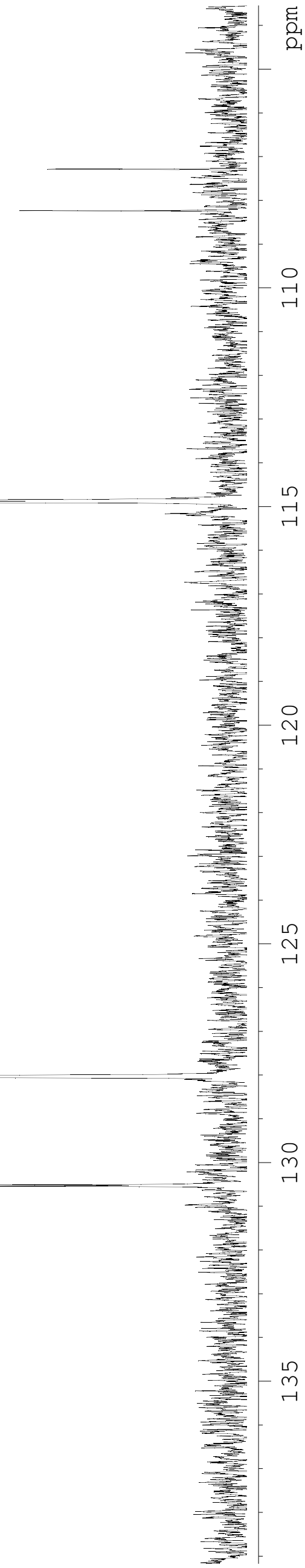


ง0ワ・78 -

LLL・ 78

o $\varepsilon \varepsilon \cdot 96$

S78 $\angle 6$

โ97.86

6 I6 66

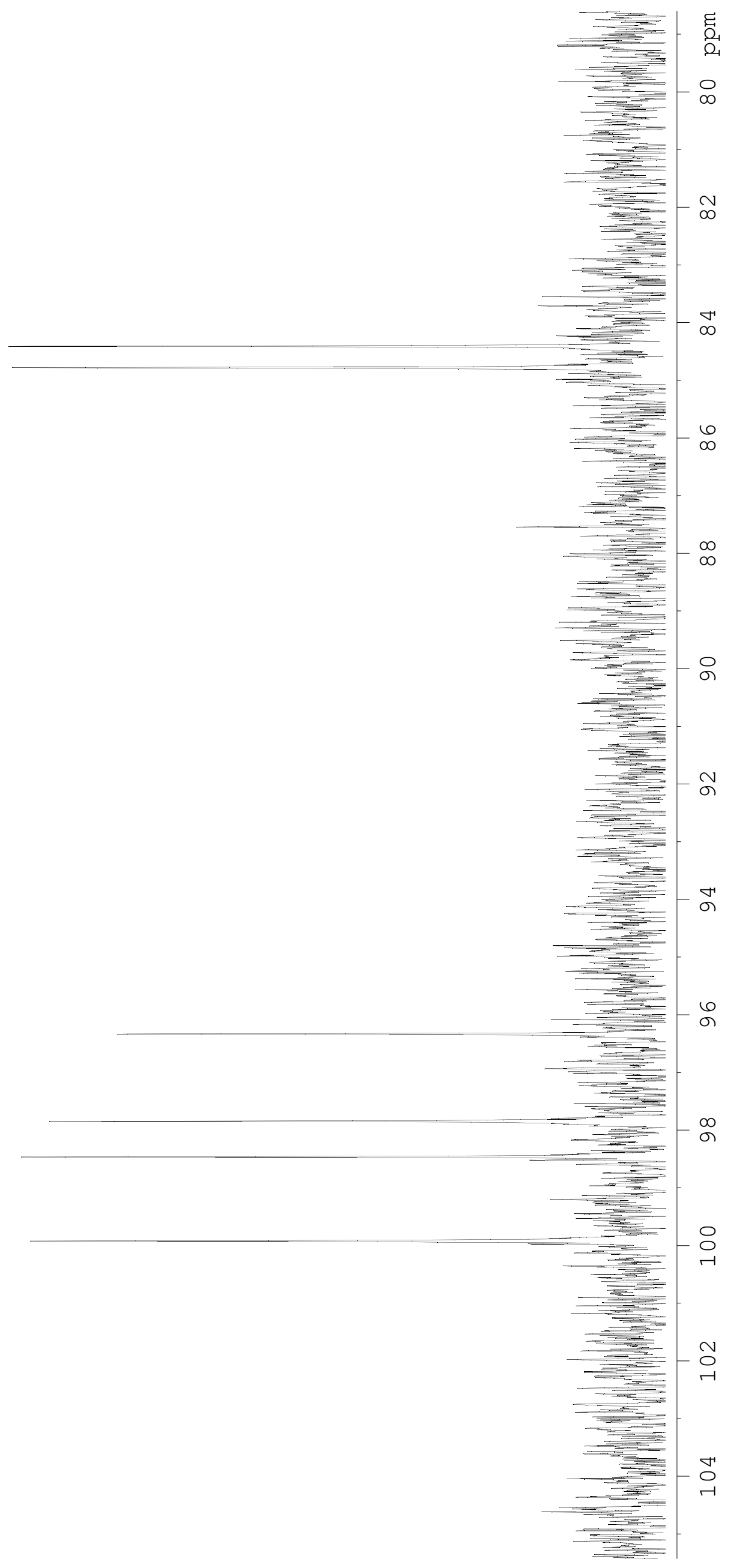




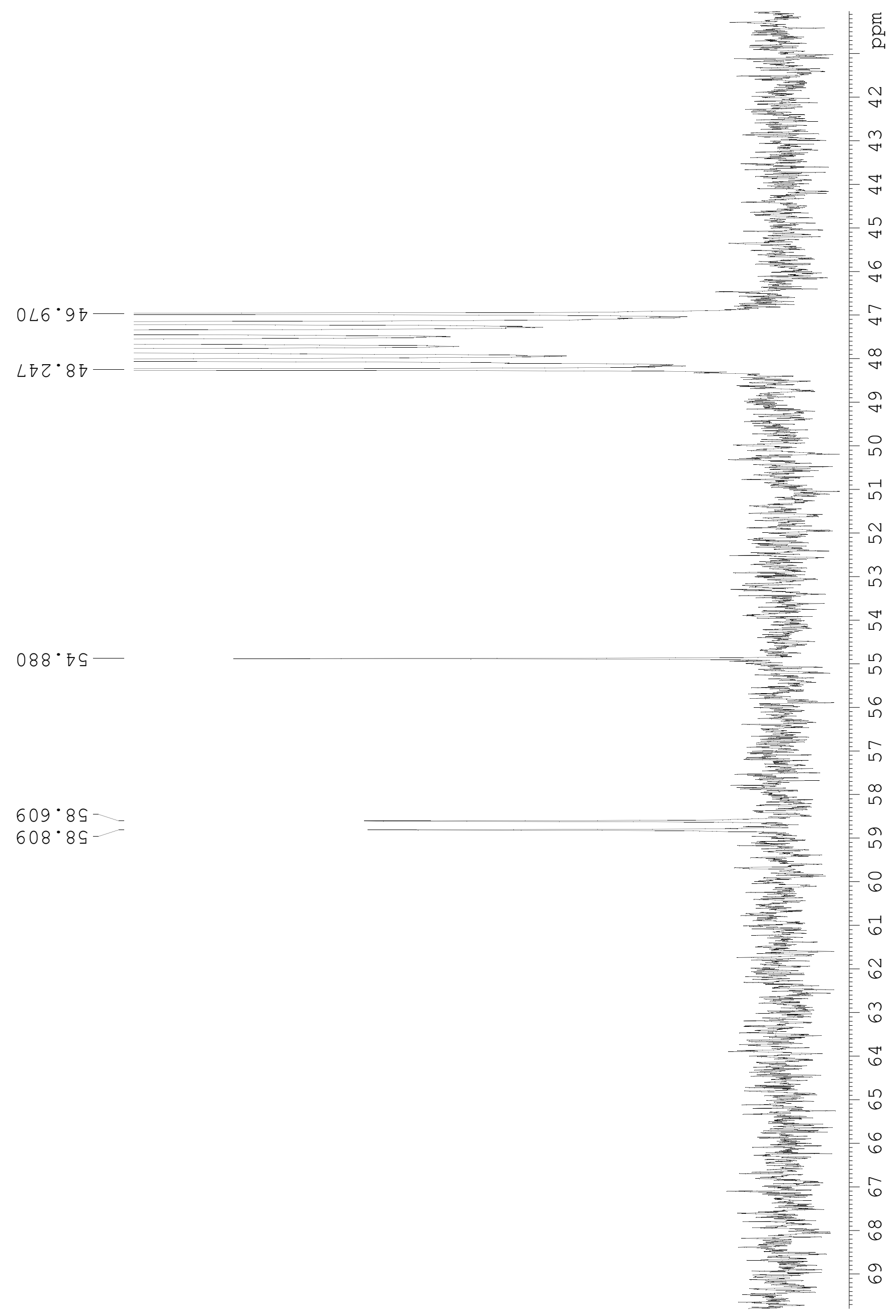


$\angle L 9^{\circ} \angle E$

I6 $8^{\circ} \angle \bar{C}$

$8 L 8 \cdot 79-$

$809^{\circ} 89$

0 โ8.89

$\angle 0{ }^{\circ}$.

$08 L \cdot$ เ8

$82 \varepsilon \cdot 96$

I $88^{\circ} \angle 6$

$\angle S D \cdot 86$

9 I6 66 -

298 $8^{\circ}$ II

ธ68 五IL

9I0.8ZI-

$\angle \oplus 0 \cdot 8 Z I$
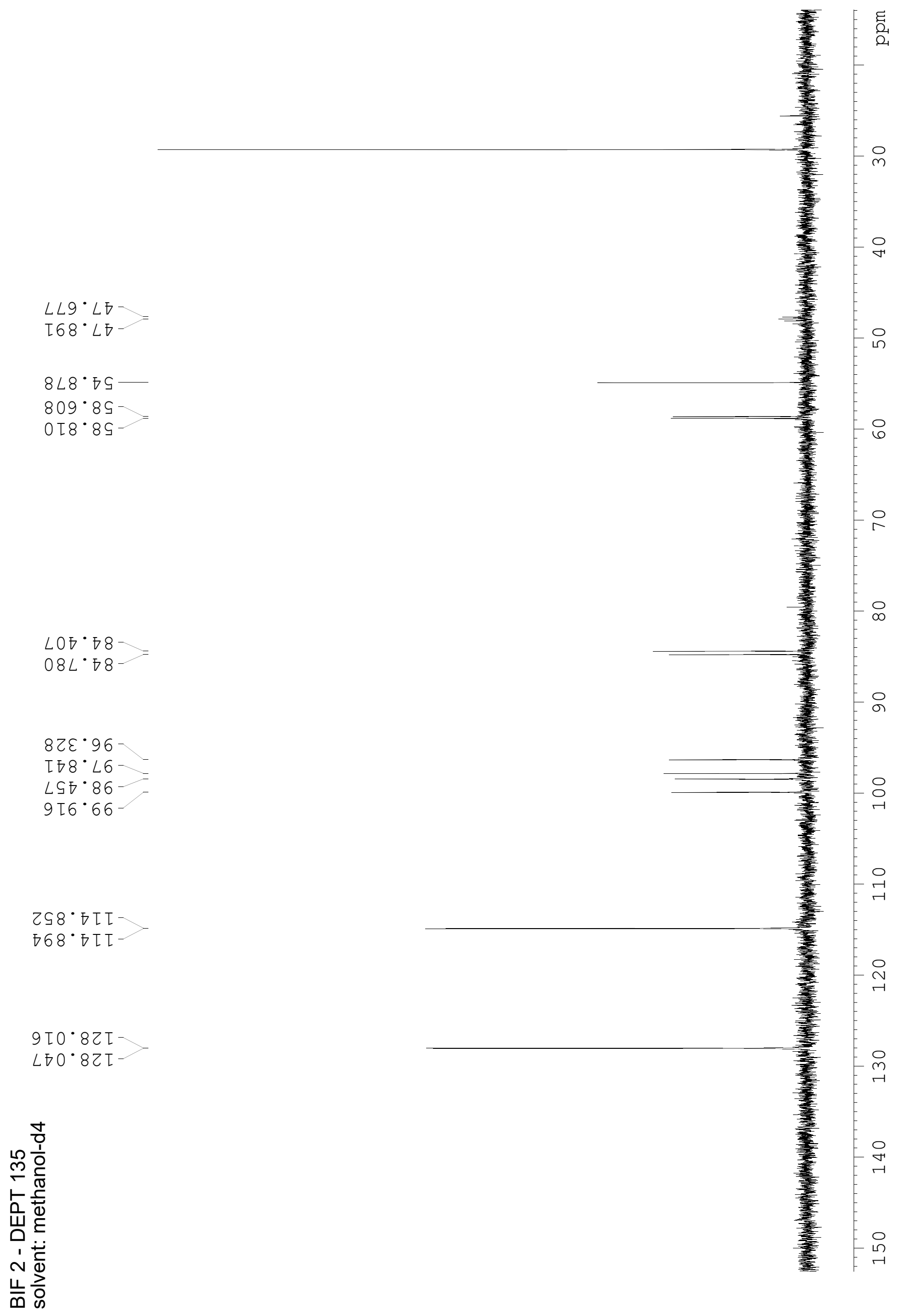
8乙ع 96

I万 $8^{\circ} \angle 6$

$\angle S 7 \cdot 86$

$9 \tau 6 \cdot 66$

29 $8^{\circ}$ 万I

$68^{\circ}$ 万II

$9[0 \cdot 8 Z I$

$\angle \mp 0 \cdot 8 Z I$

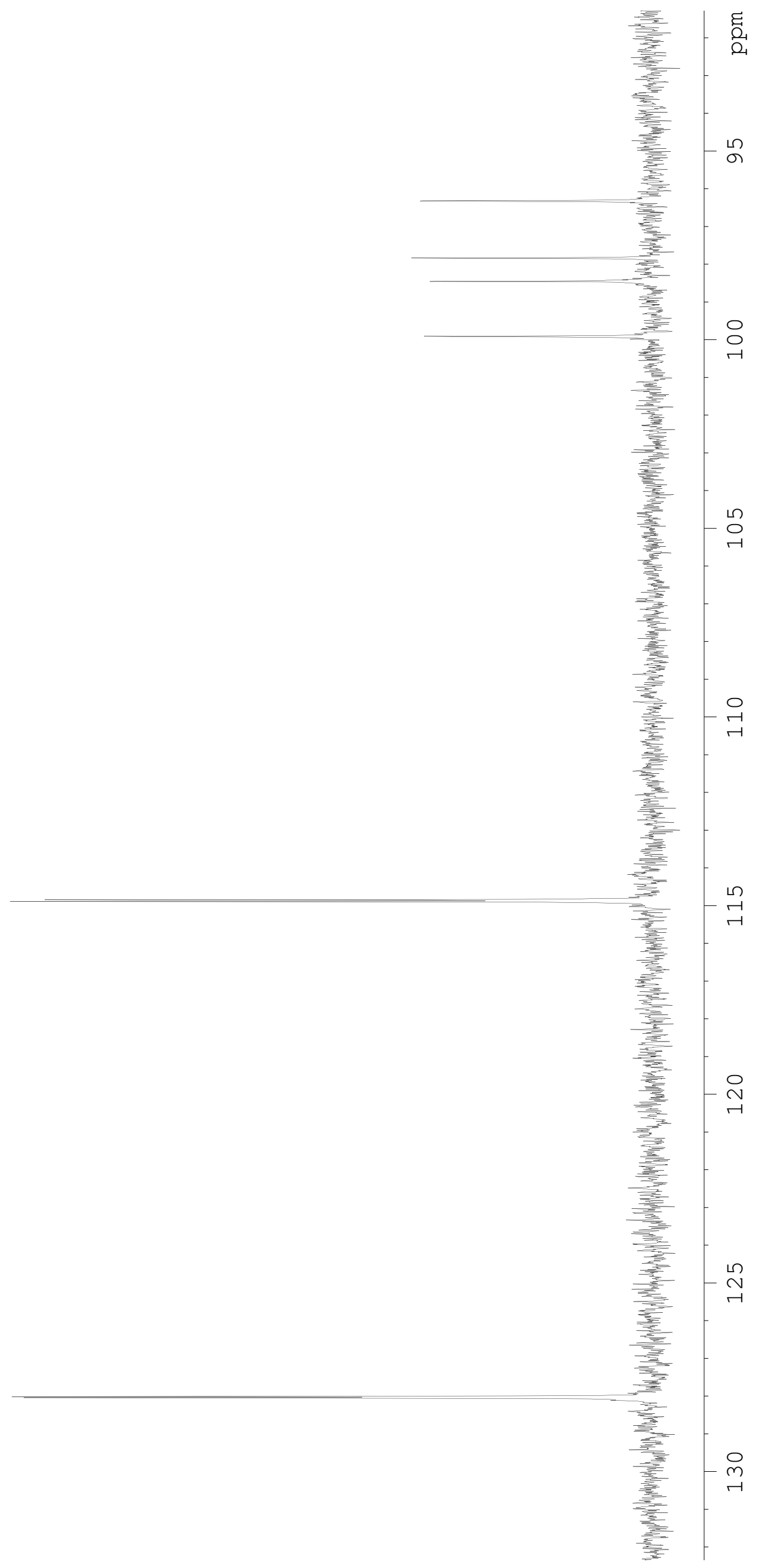


$268^{\circ} \operatorname{LD}$

$848^{\circ}$ TS

$809 \cdot 89$

0 [8.89

$\angle 07 \cdot 78$

$08 L \cdot 78$

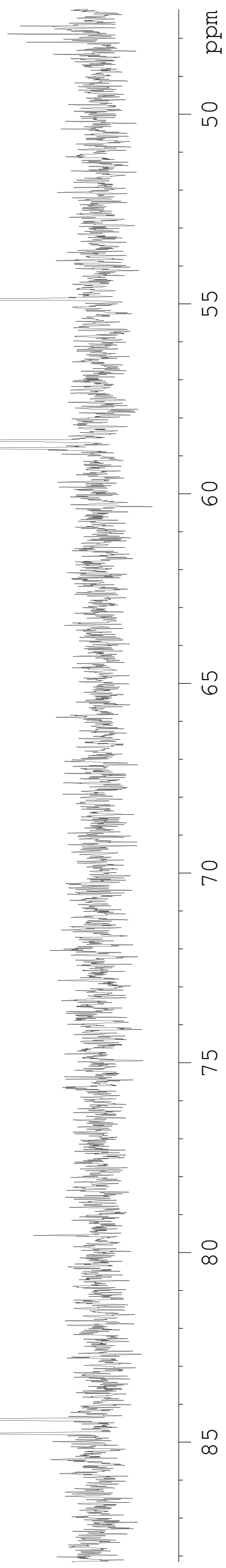




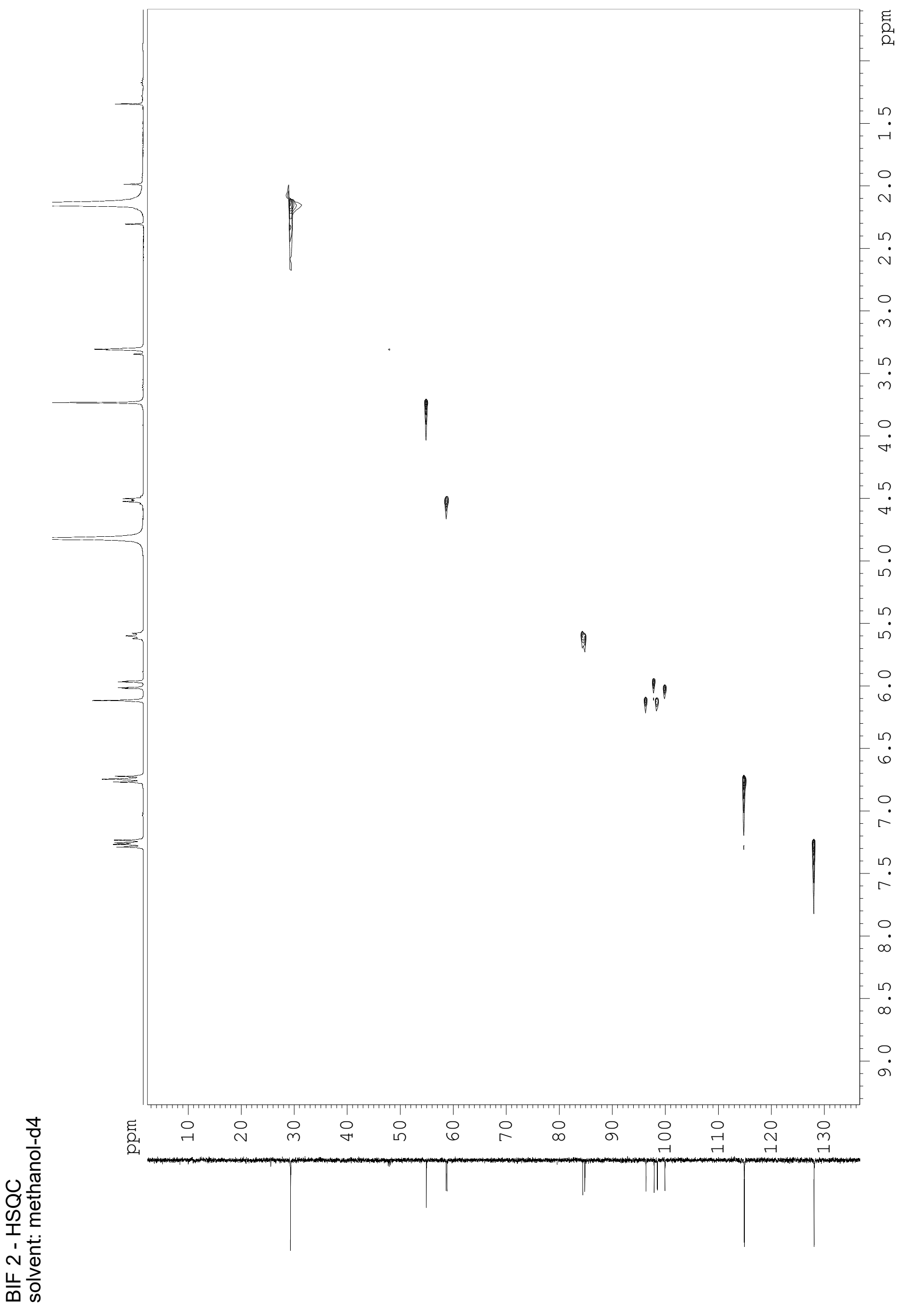




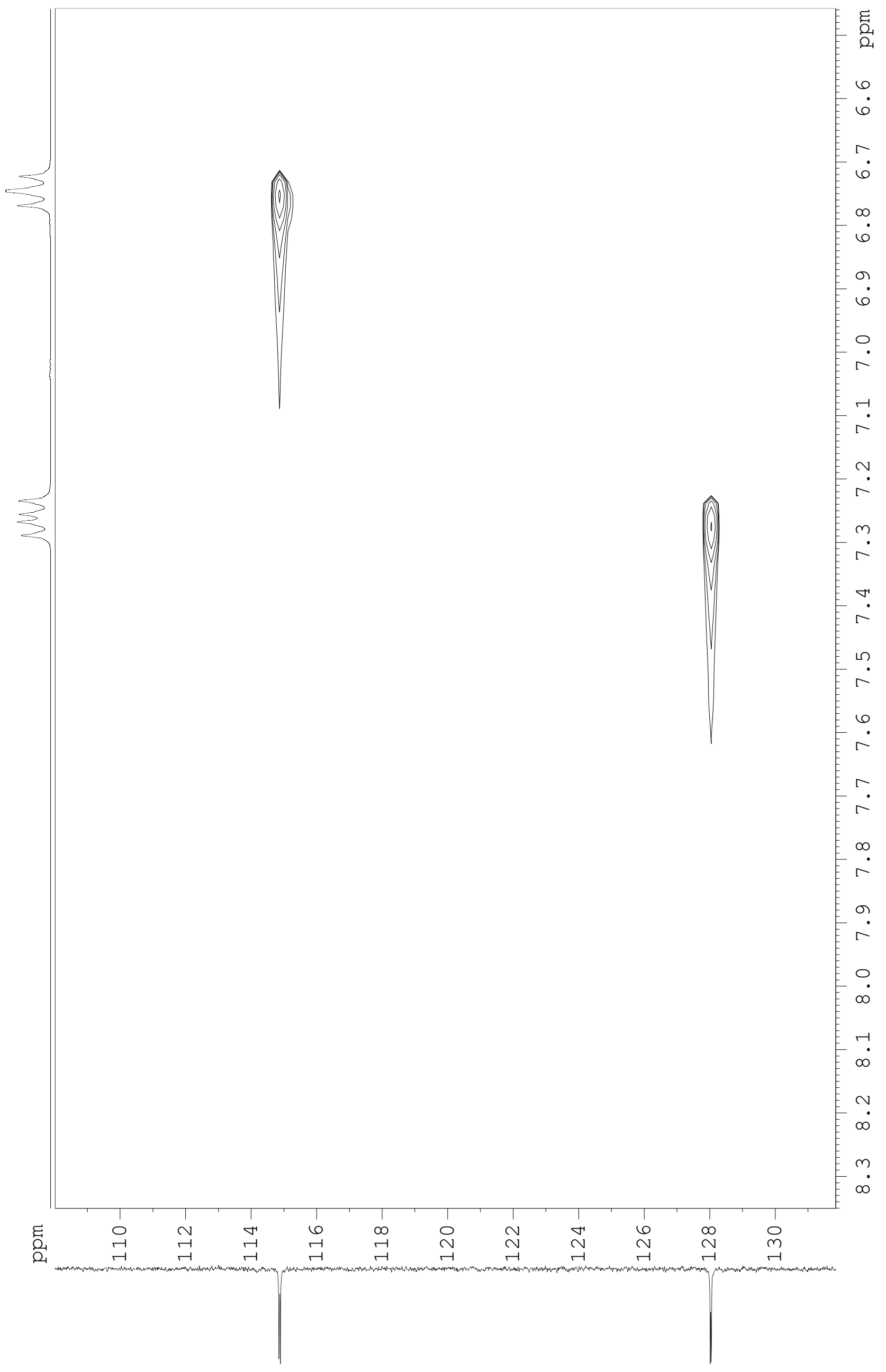




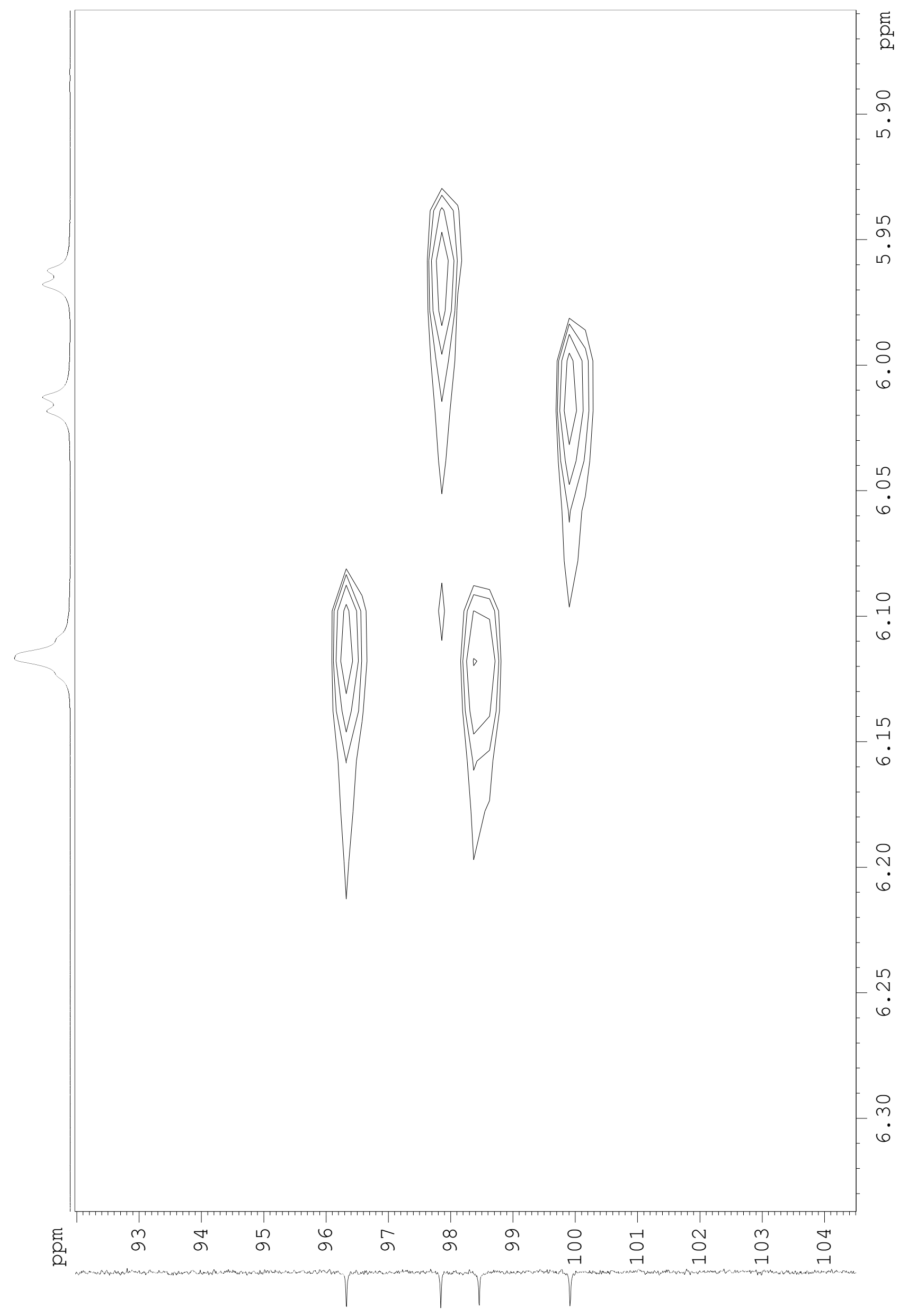




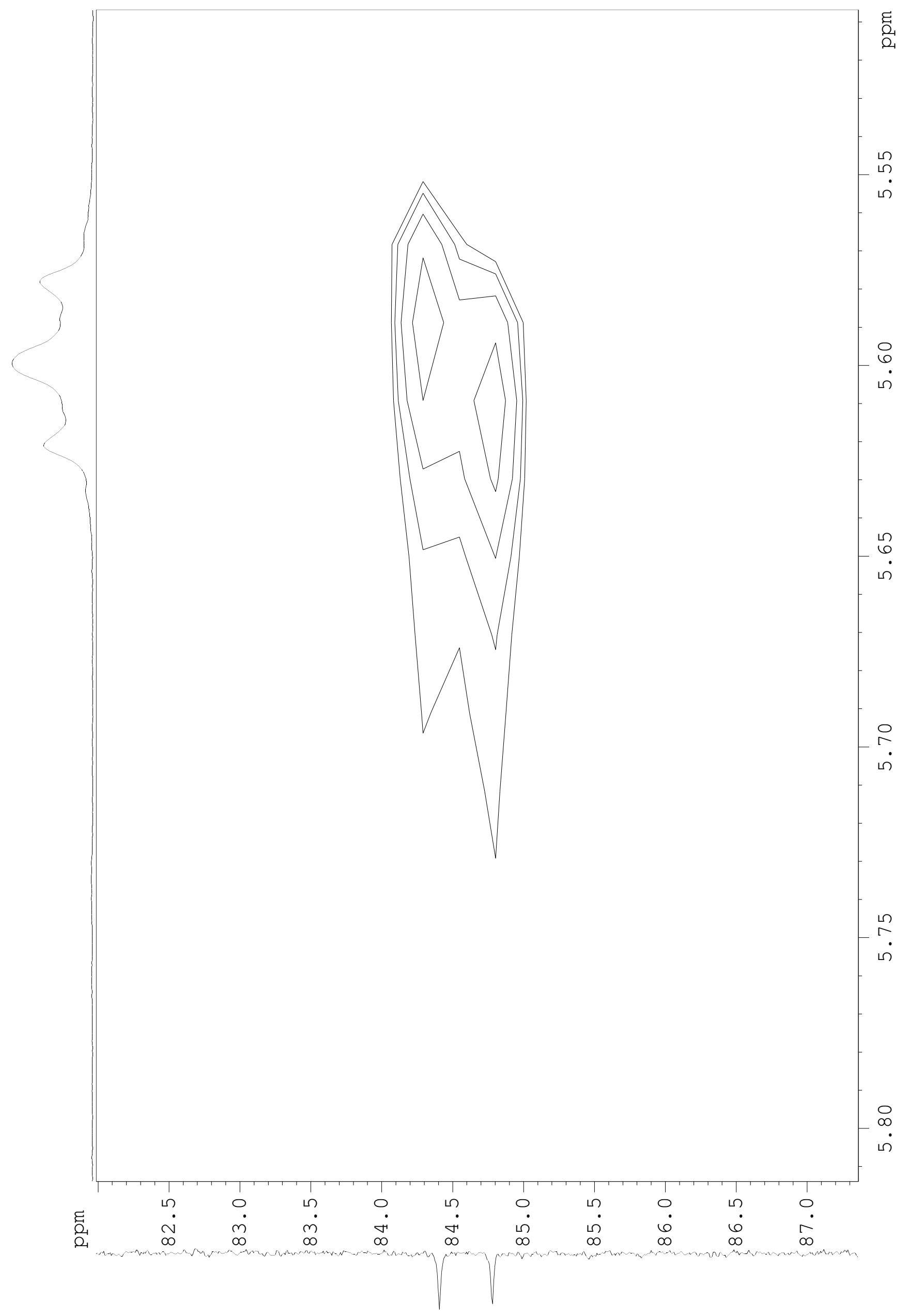




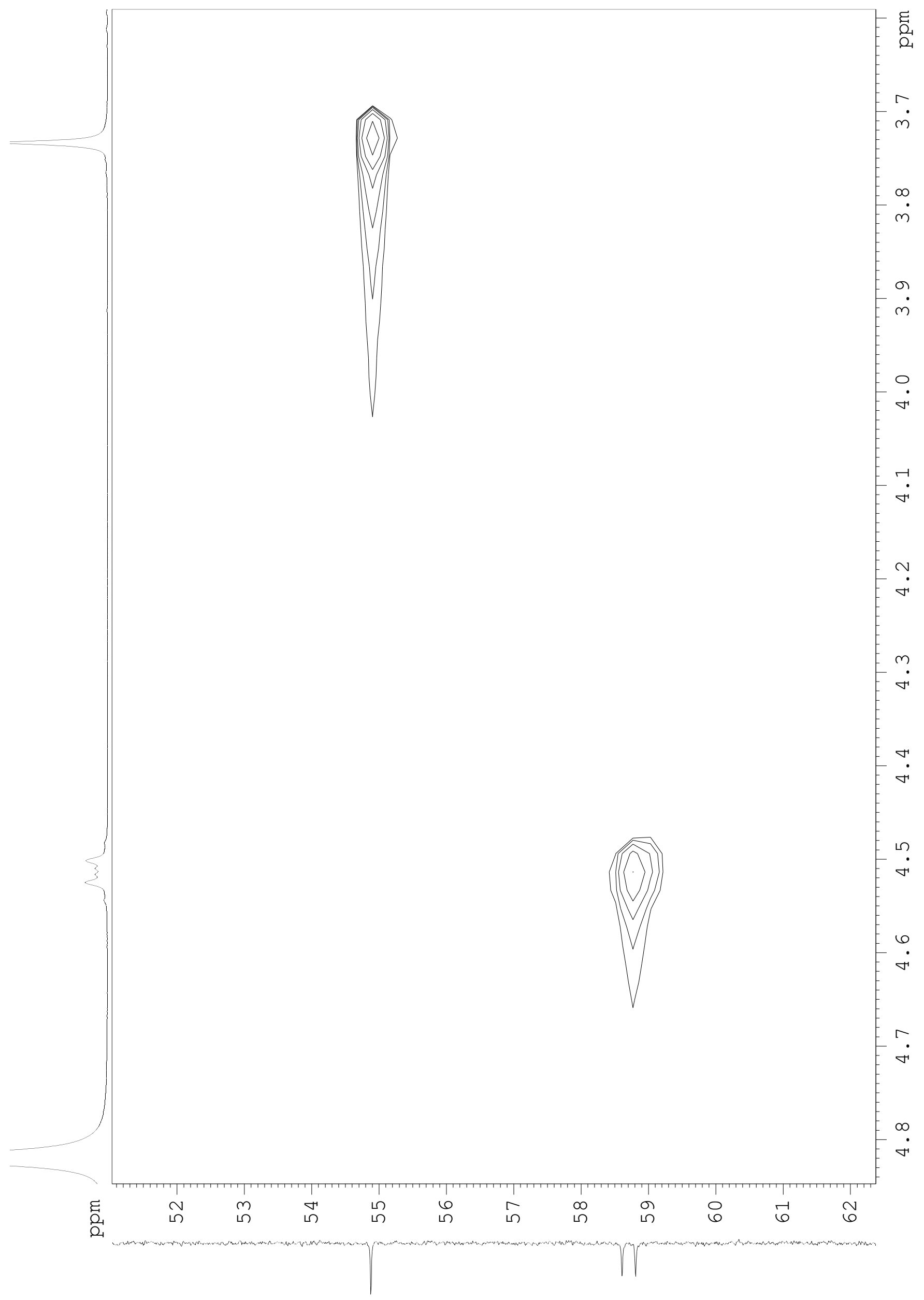




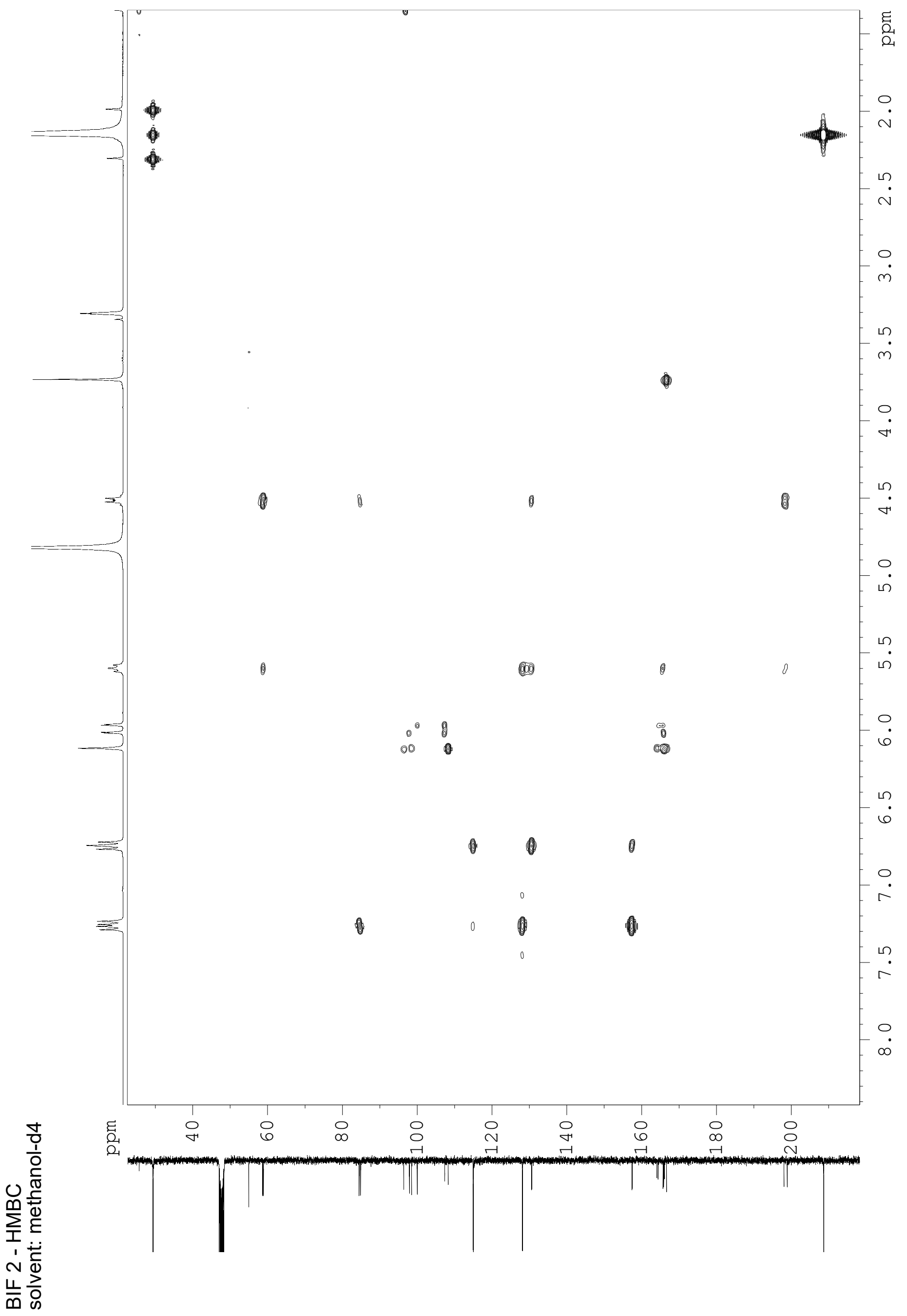




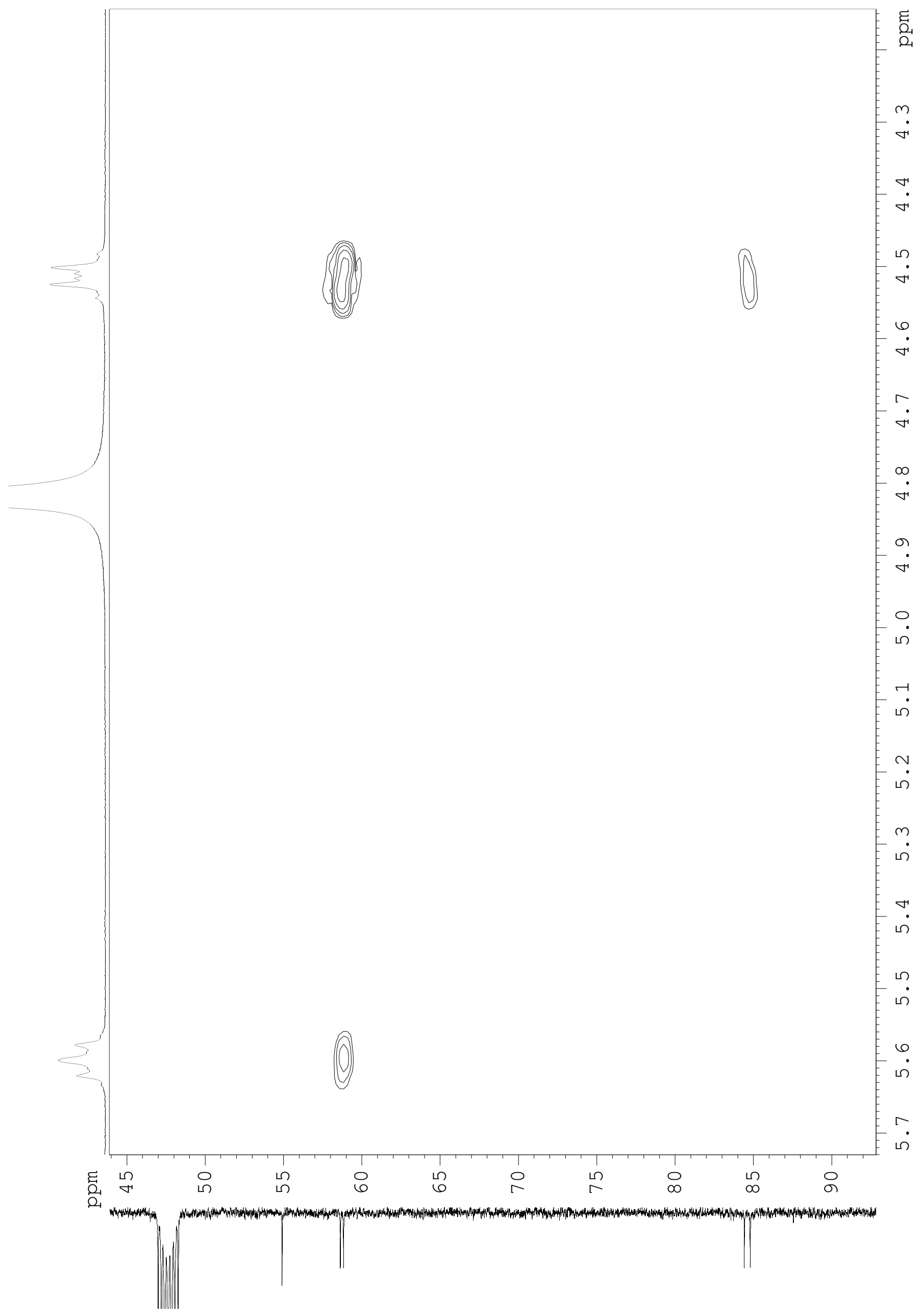




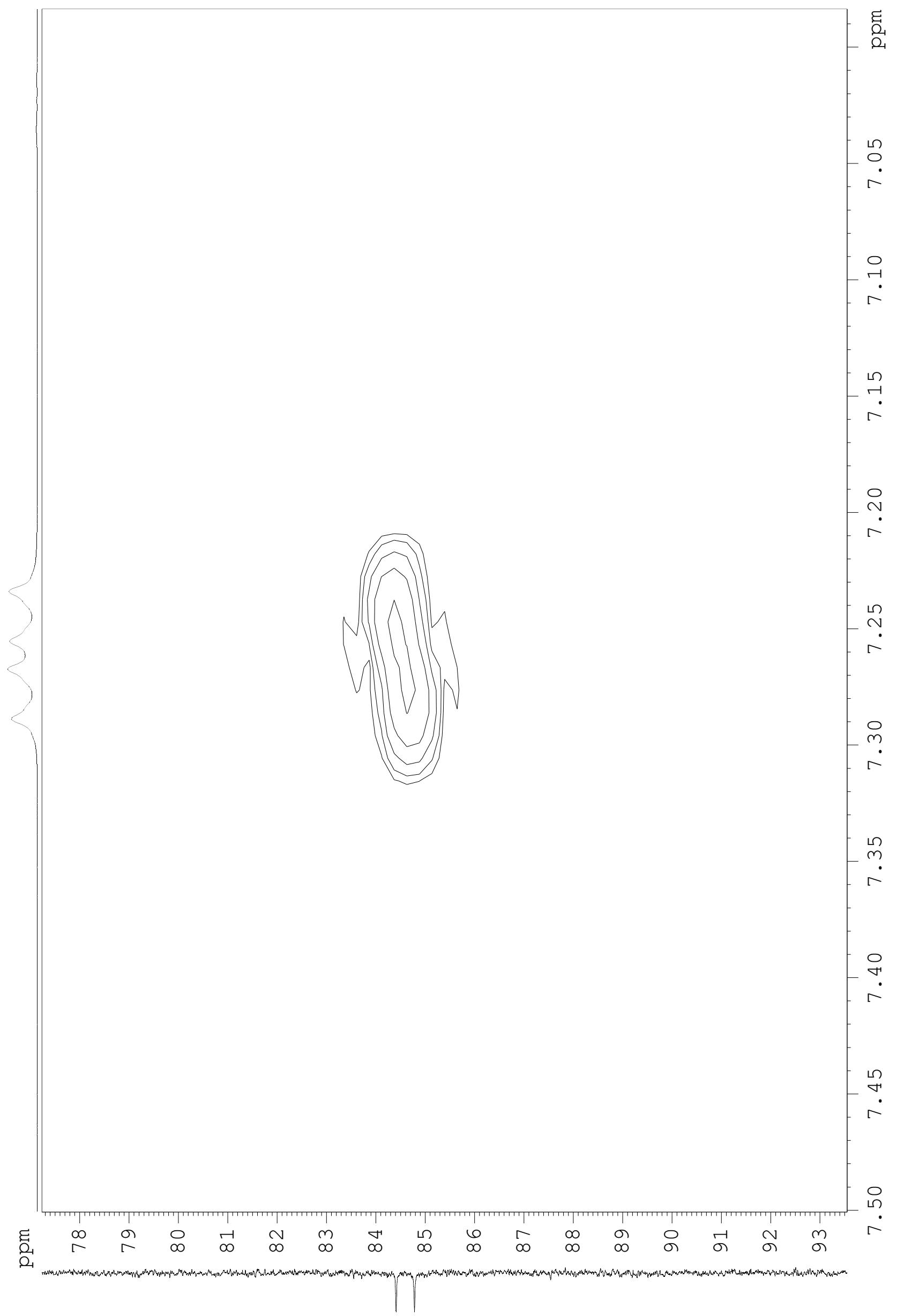




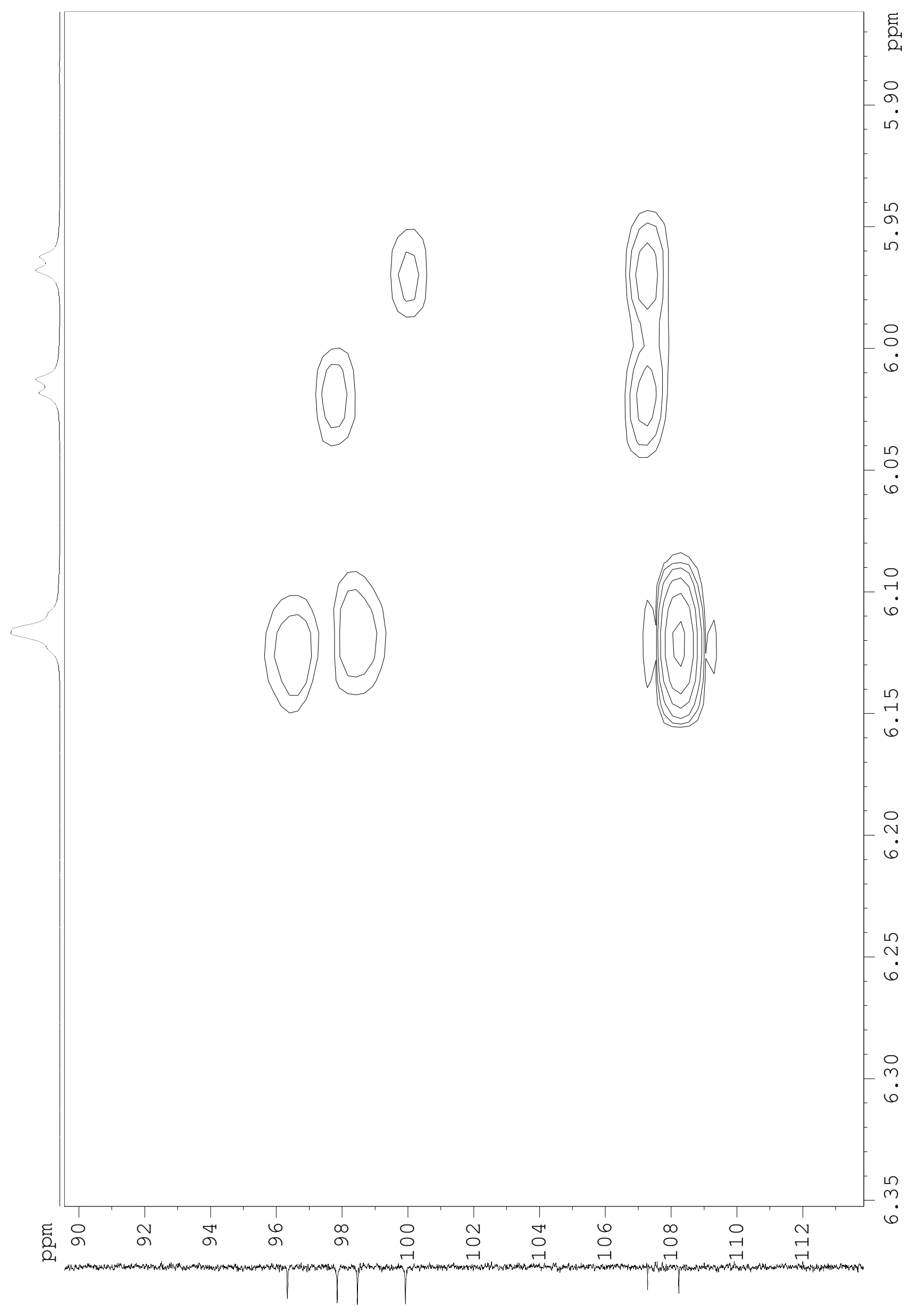




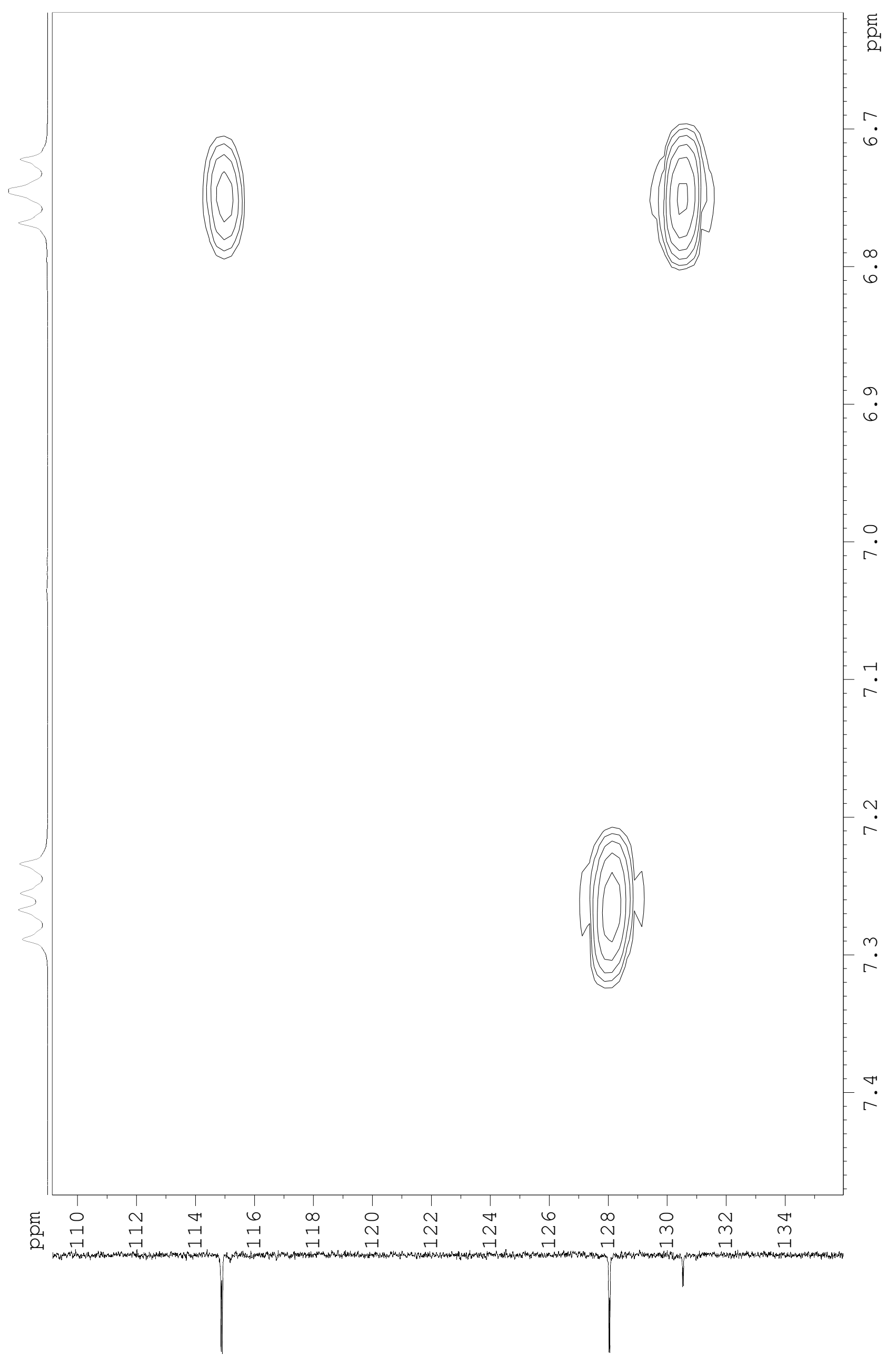




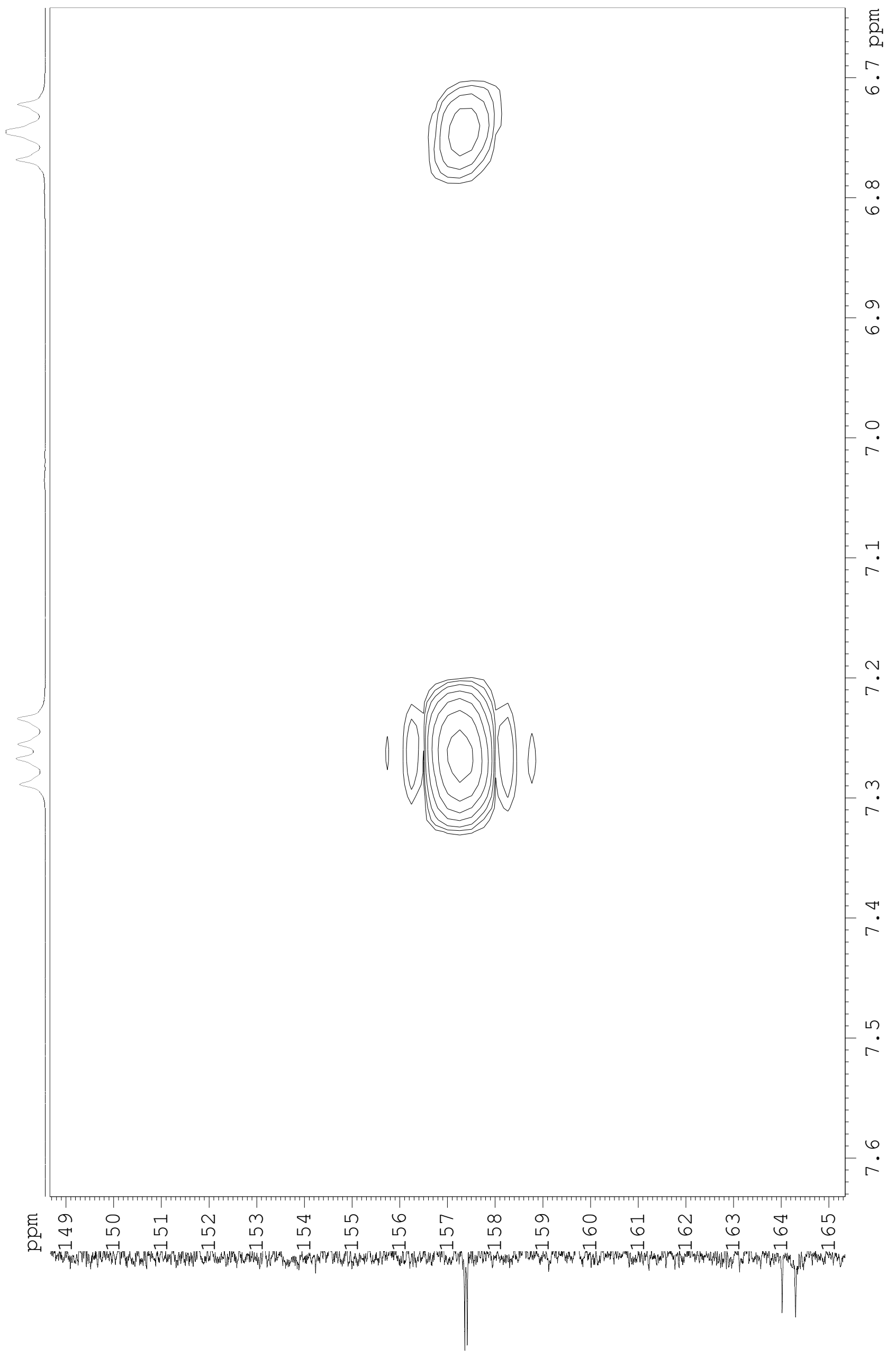




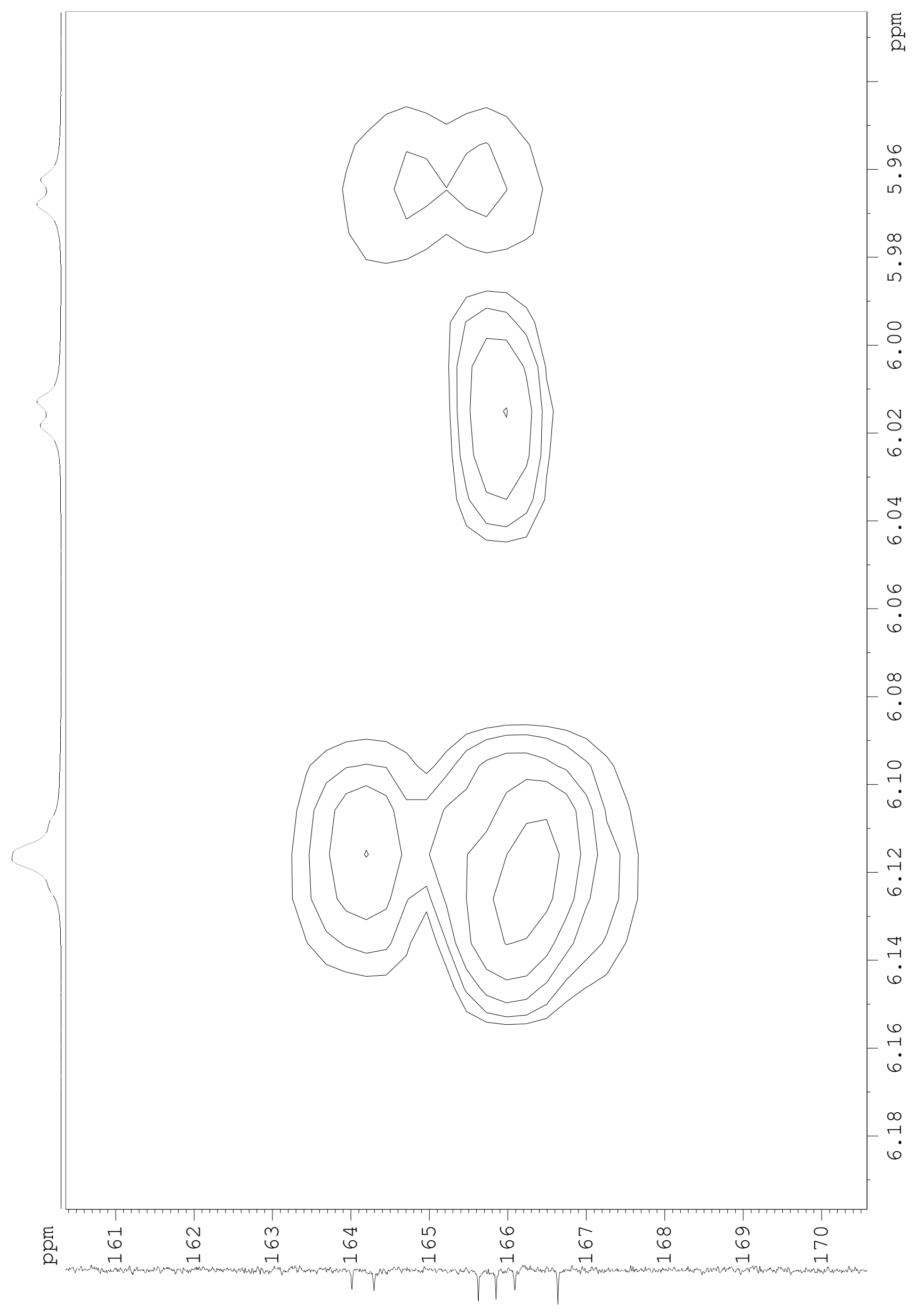




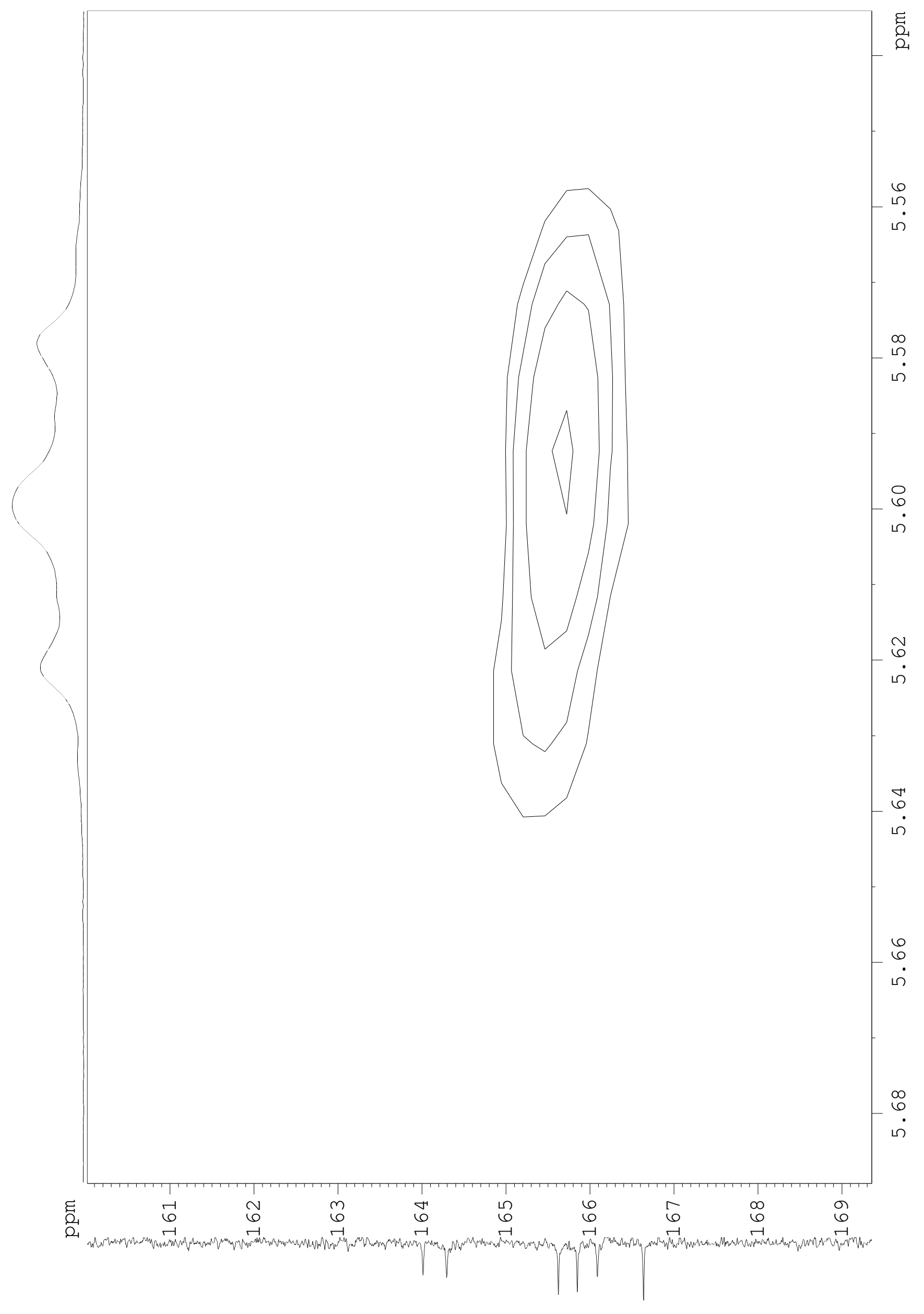




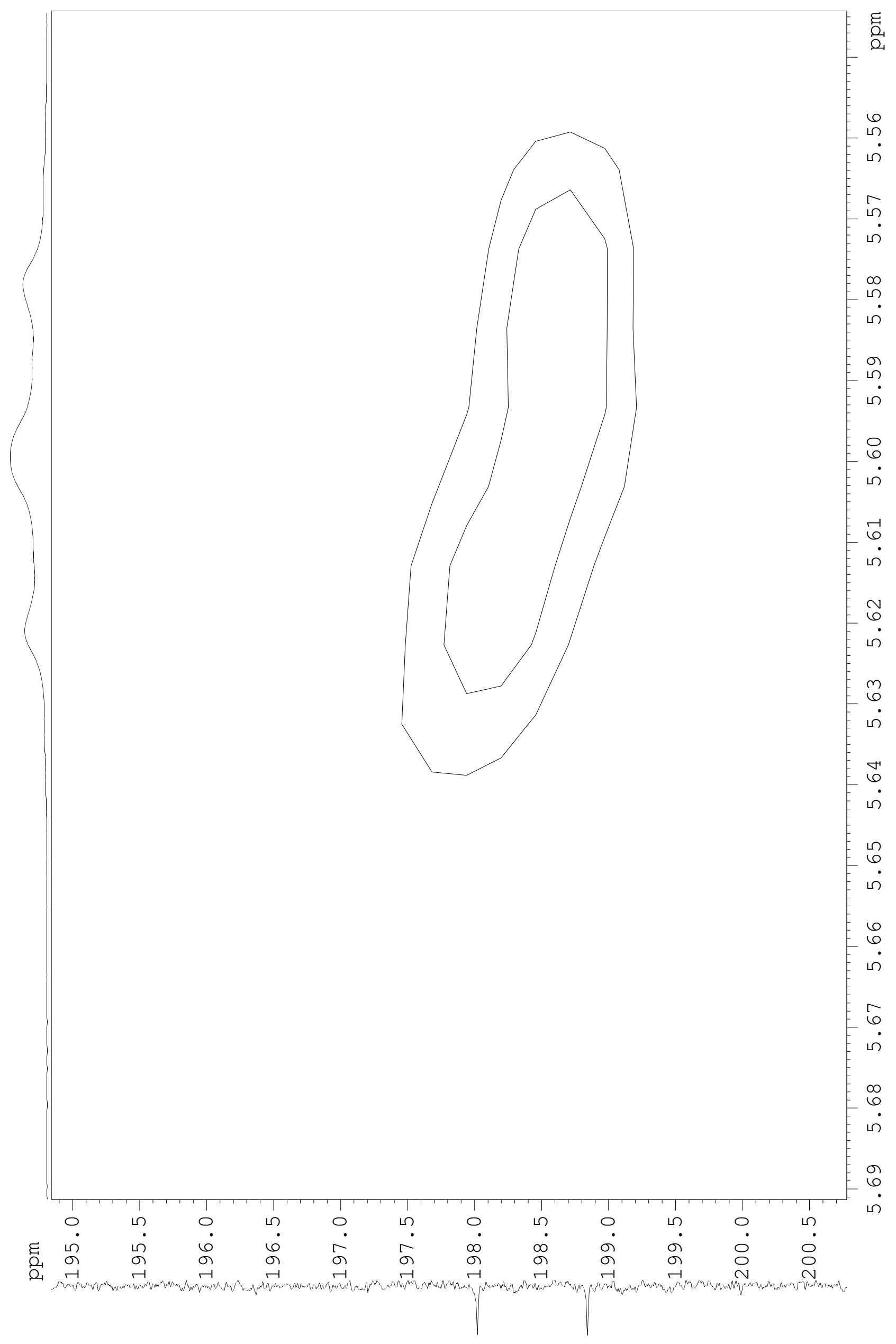




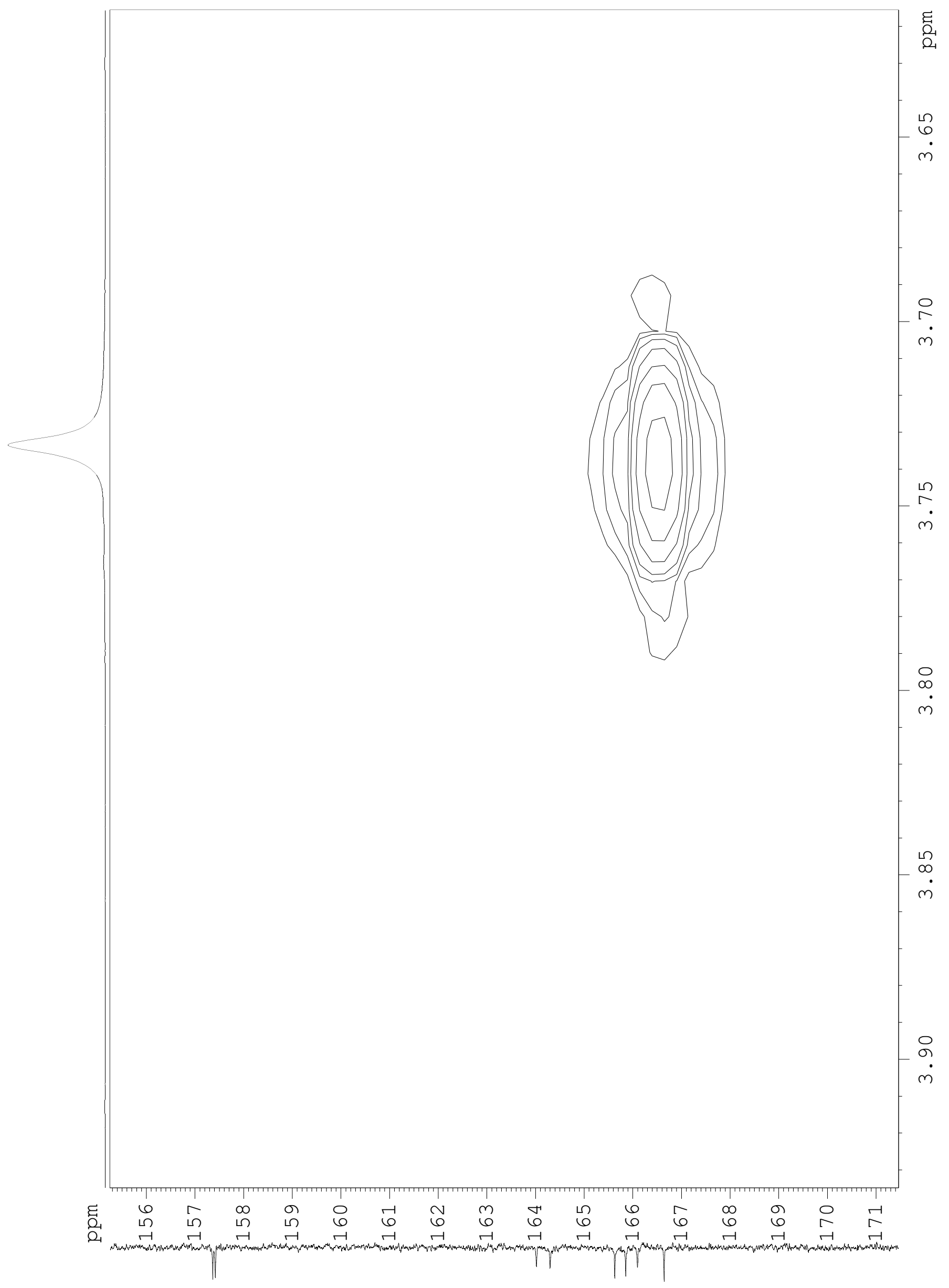




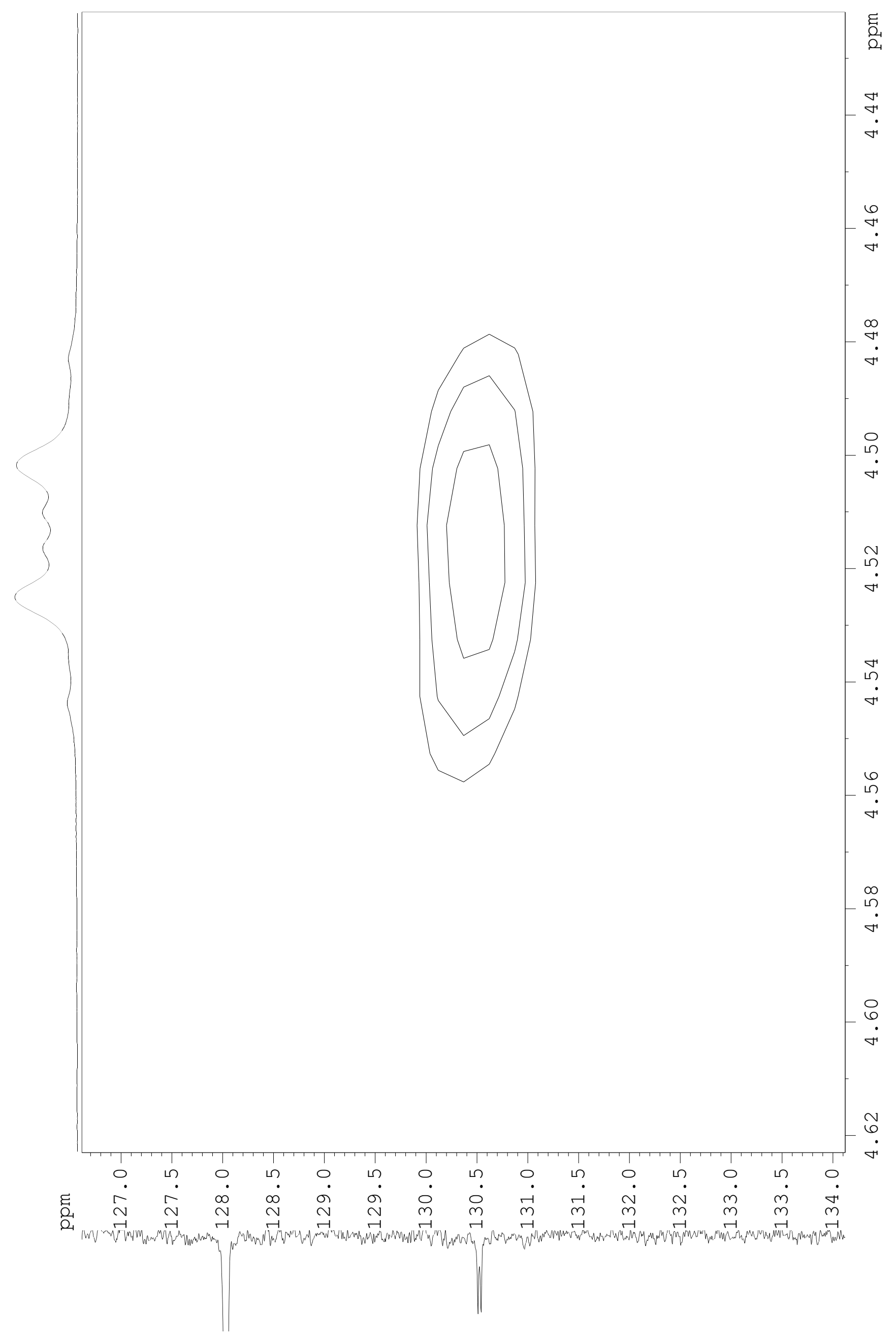




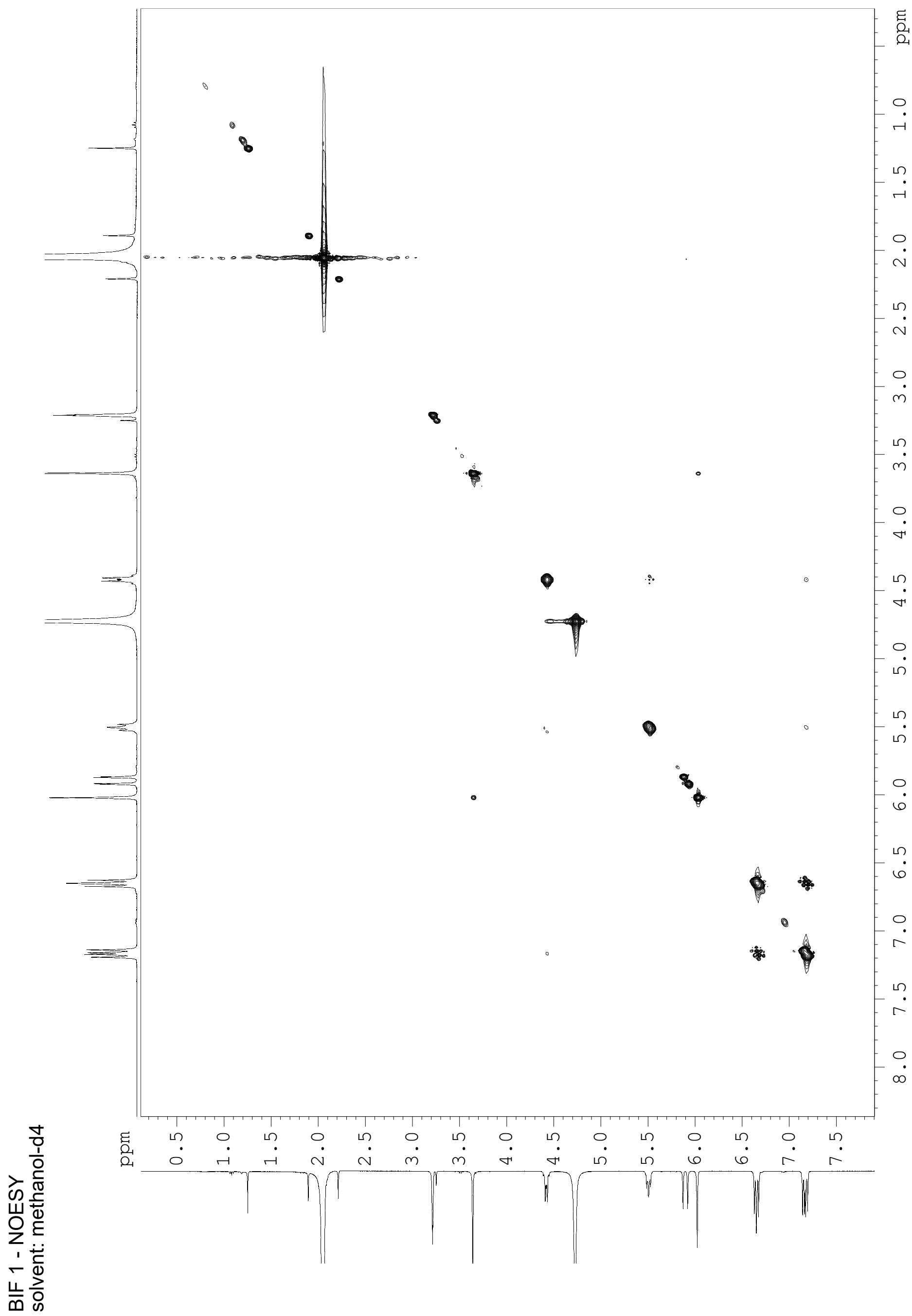




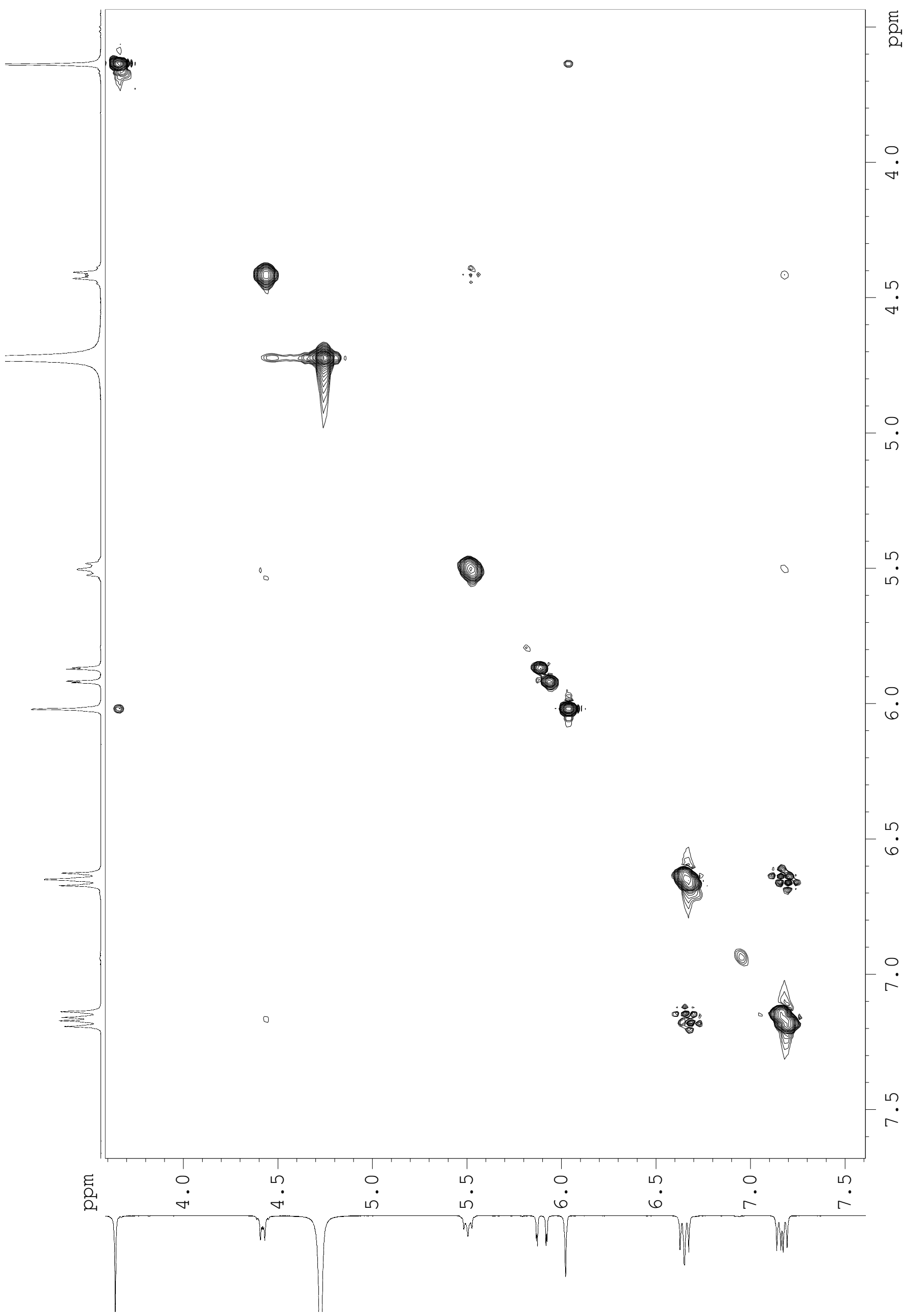




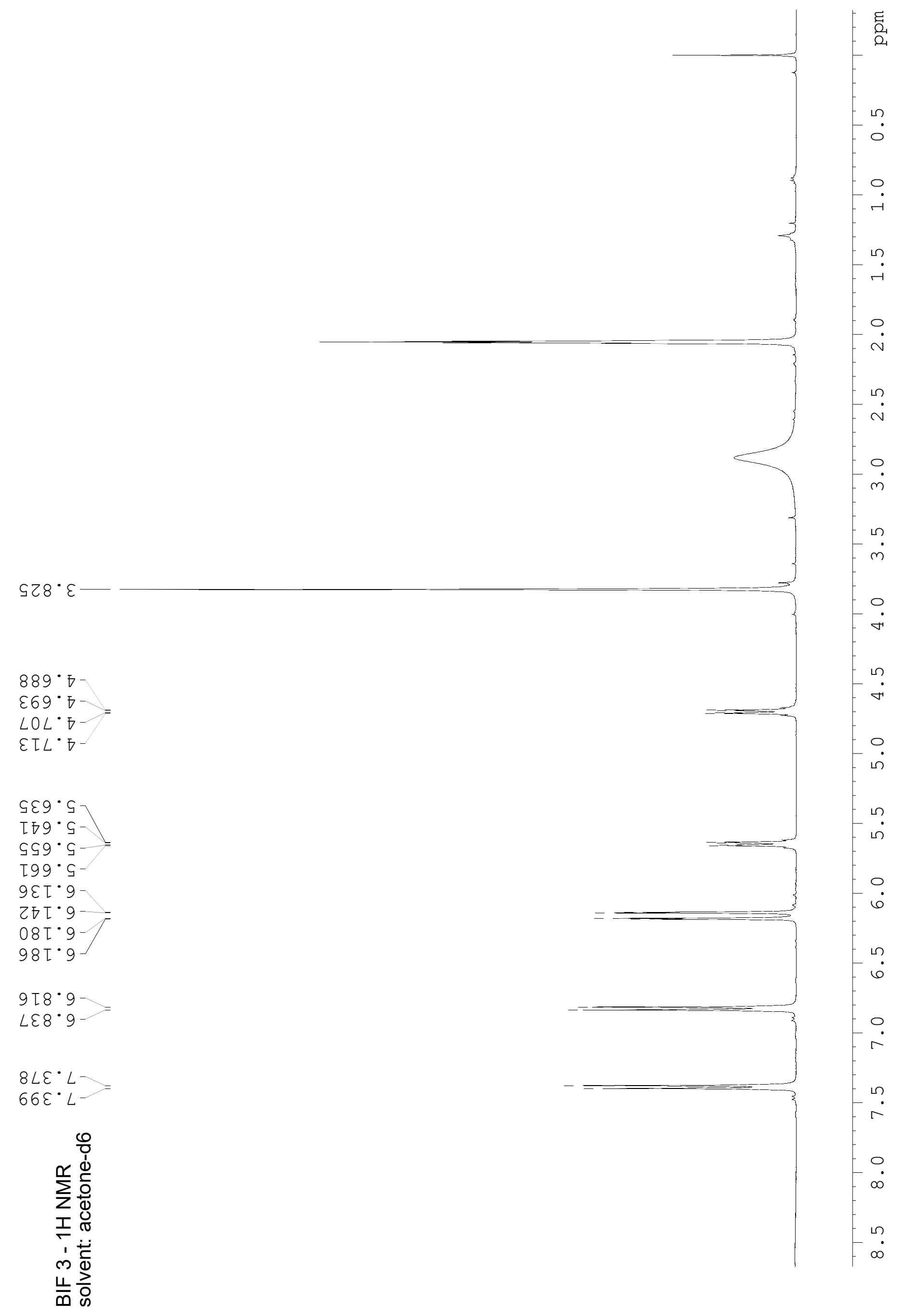


$9 \varepsilon[\cdot 9-$

乙๐ $\cdot 9$

$08 I \cdot 9$

$98 I \cdot 9-$
$9[8 \cdot 9$

LE 8 9

$8 L \varepsilon^{\bullet} L$

$66 \varepsilon^{\circ} L-$
ह્ڤૂ

․

$\dot{6}$

$\stackrel{r}{6}$

ம

6
6

ம.

$+\infty$

-

$\stackrel{0}{\circ}$

다․

$r$

$E N$

r

m

r

$r$

$r$

ก

다․ 
$889^{\circ} \overline{7}$

$\varepsilon 69^{\circ} \overline{0}$

$\angle O L{ }^{\circ} \bar{D}$

$\varepsilon \tau L \cdot \sigma-$

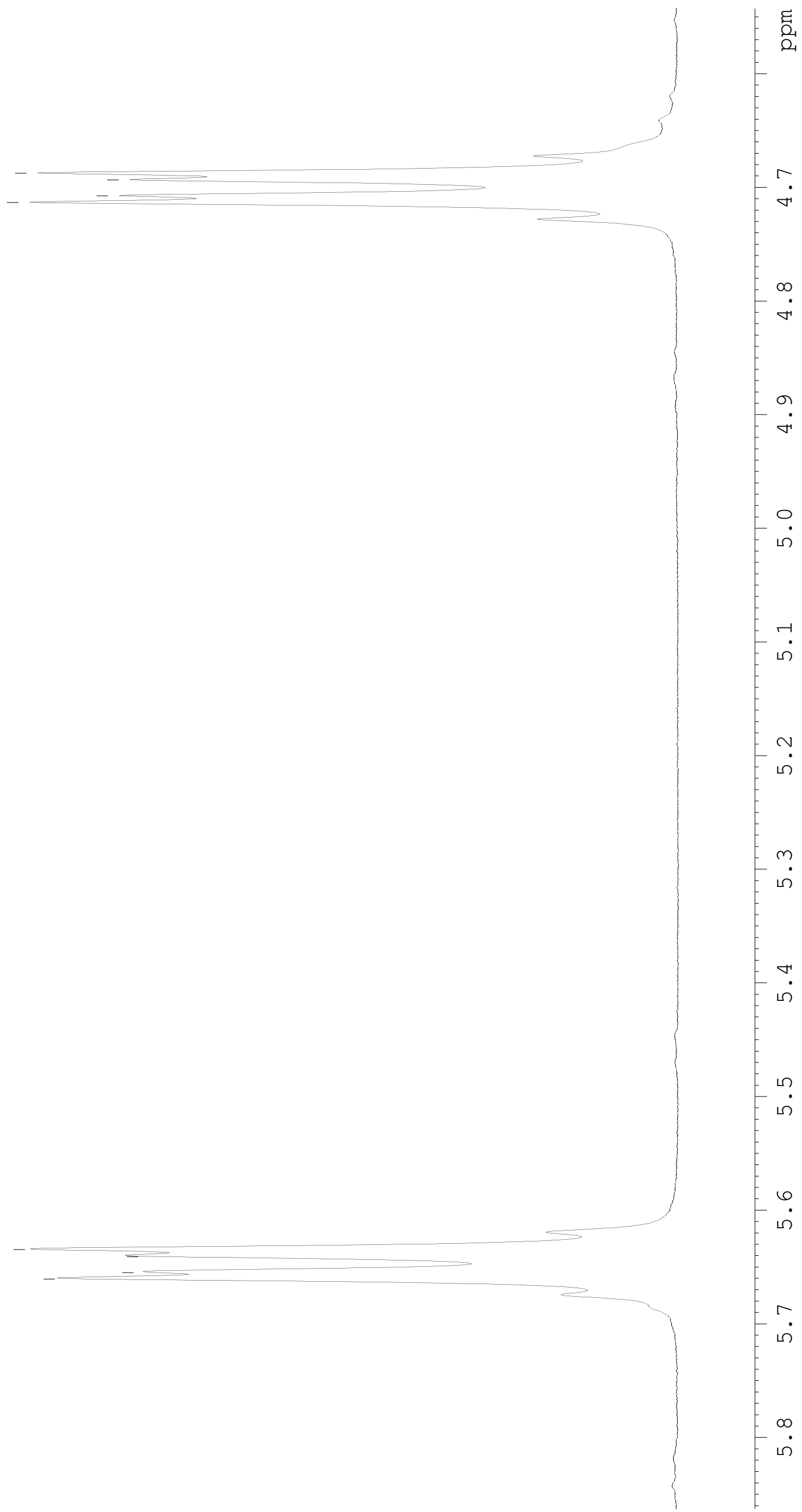

GEg $G$

โฤ9. 9

ธ $99^{\circ} \mathrm{S}$

$\left[99^{\circ} \mathrm{S}^{-}\right.$
ڤి

$\diamond$

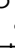

$\stackrel{\circ}{\circ}$

N

ก

m

$\stackrel{r}{\circ}$

เ

$\ddot{0}$

.

$\infty$.

ம 


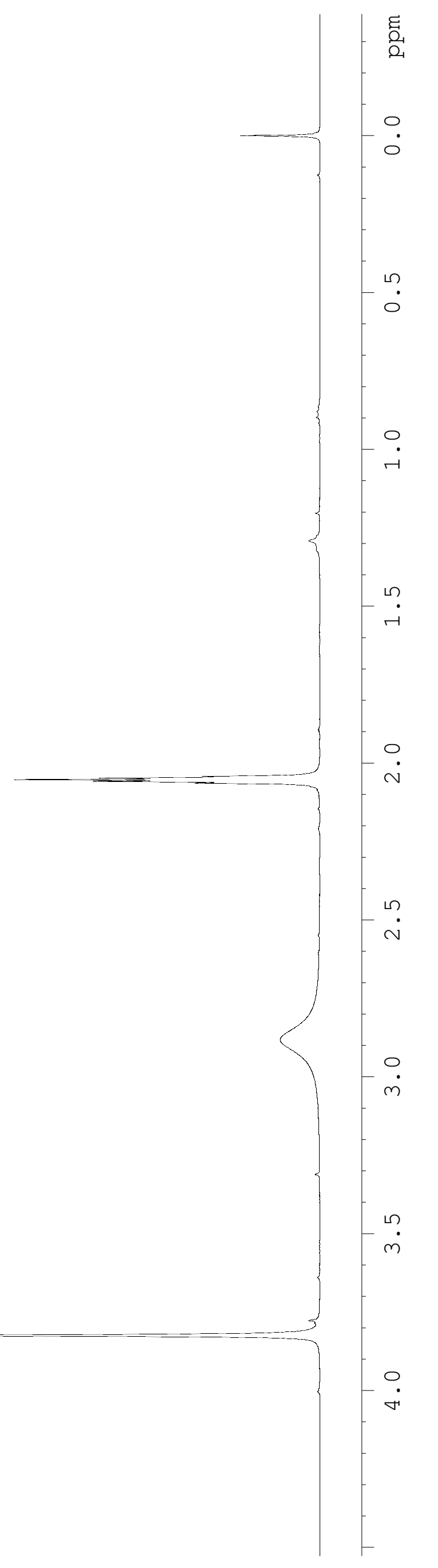

$\varsigma 乙 8 \cdot \varepsilon$ 


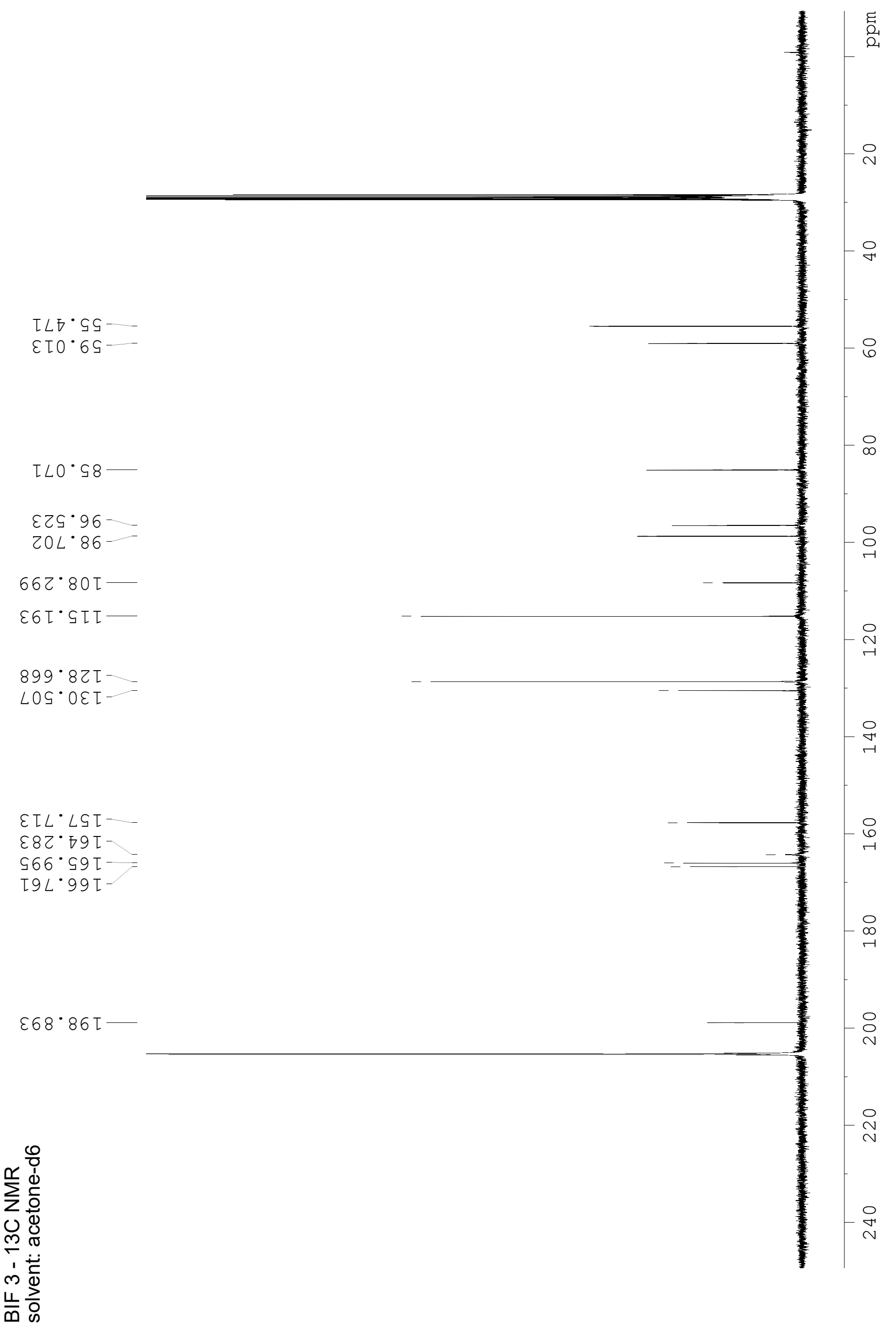




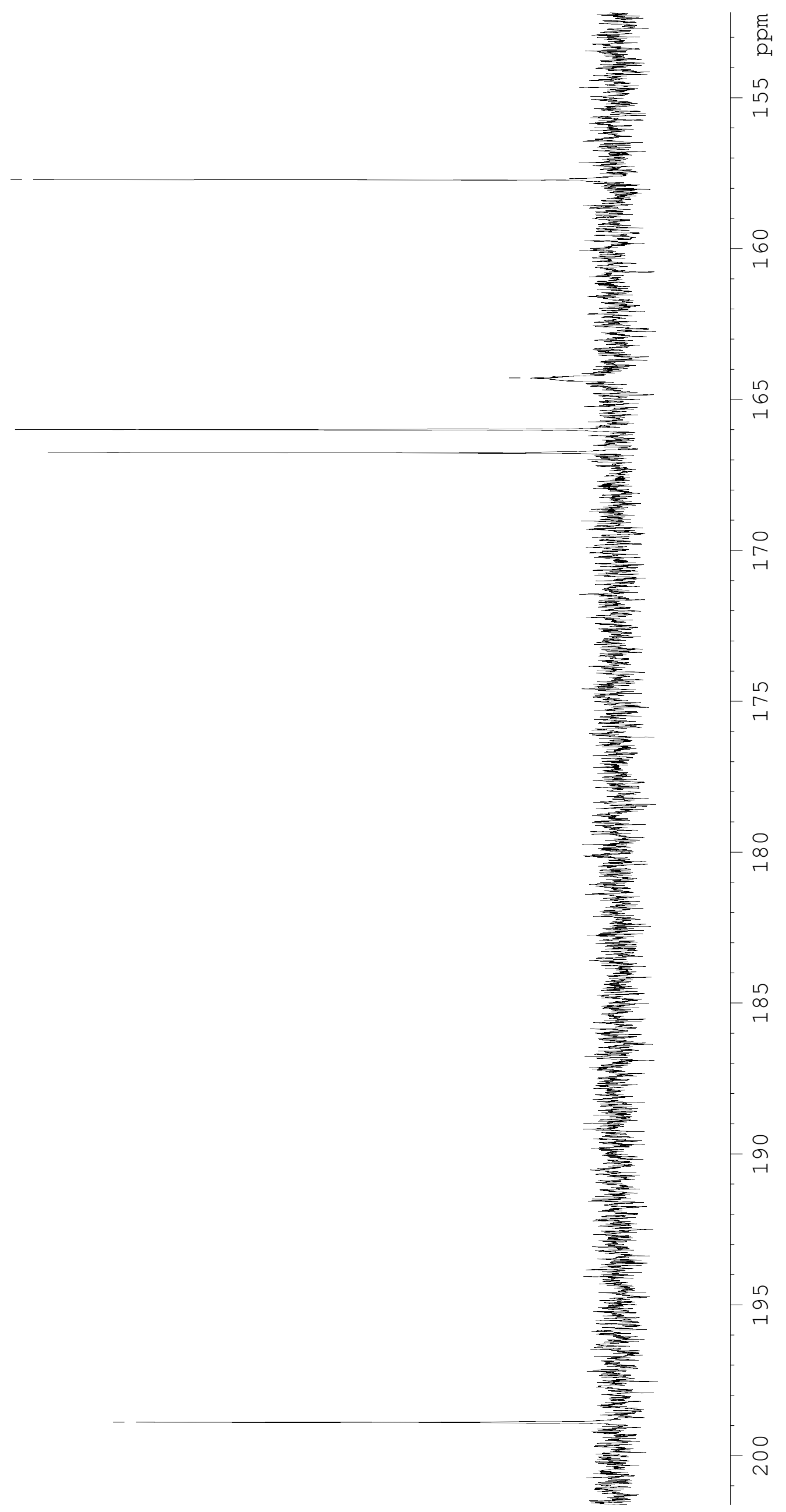


हZ५.96

$20 L \cdot 86$

$66 \tau \cdot 80 \mathrm{I}$

$\varepsilon 6 I \cdot S I T-$

$899^{\circ} 8 Z I-$

$\operatorname{LOS}^{\circ}$ OEI

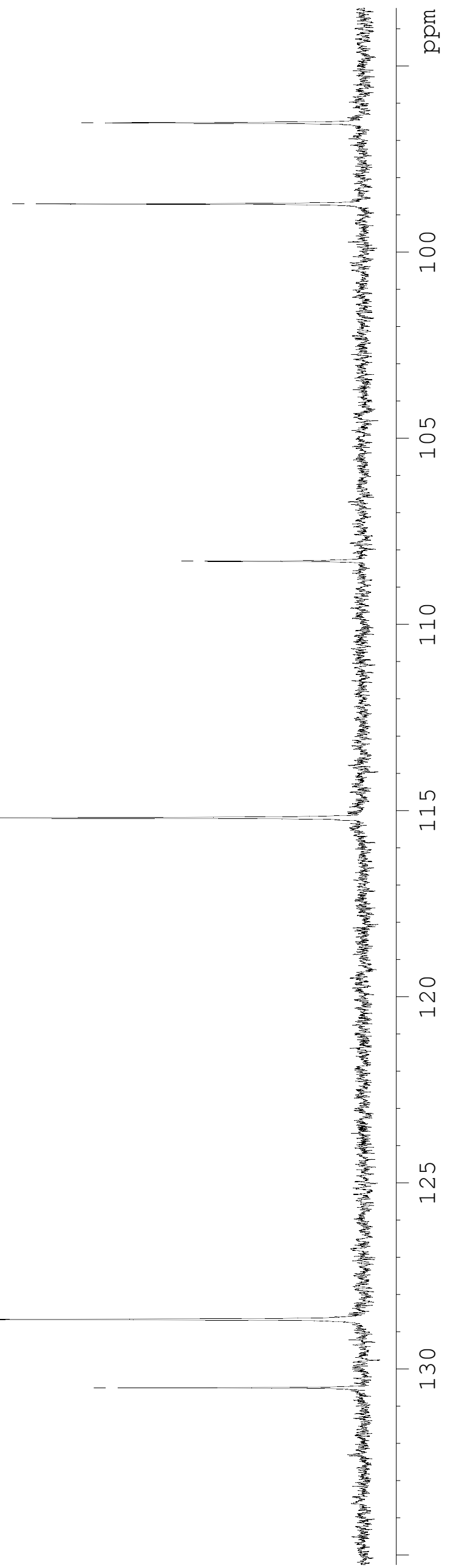




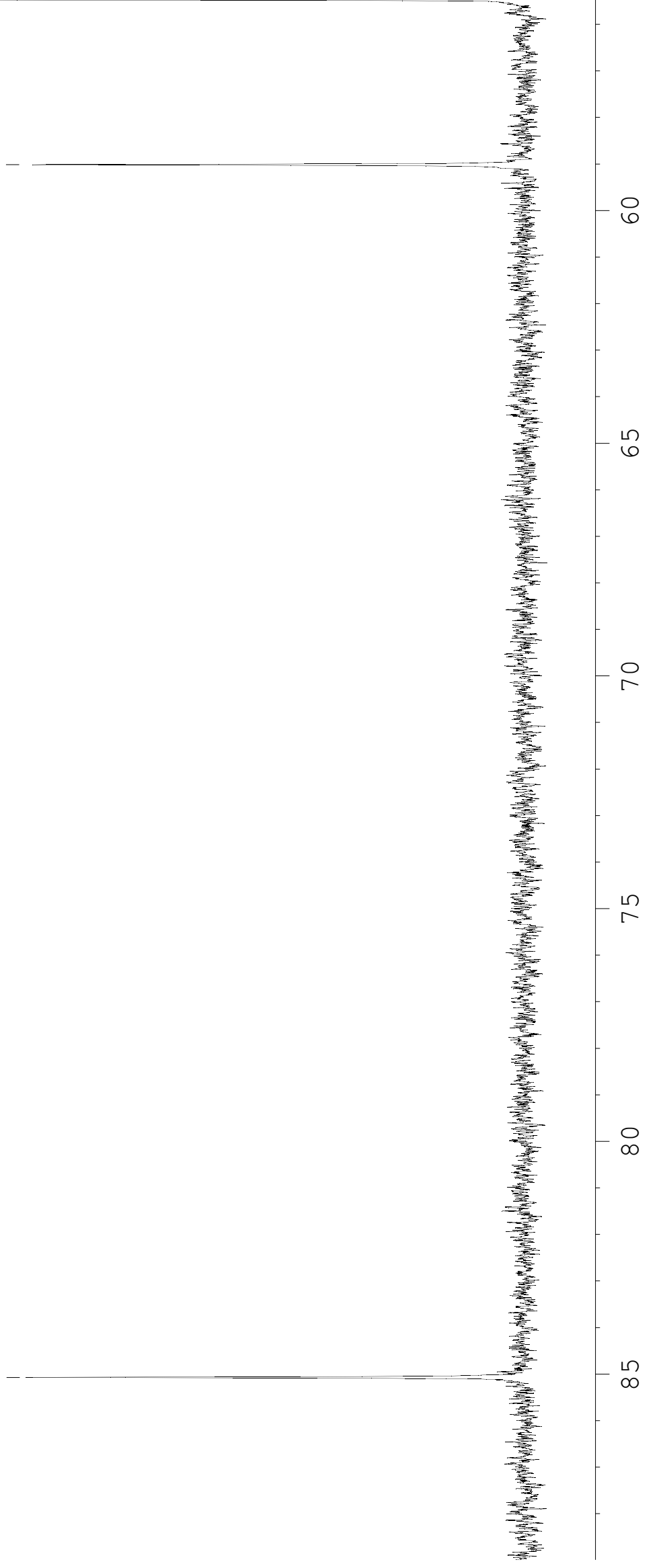




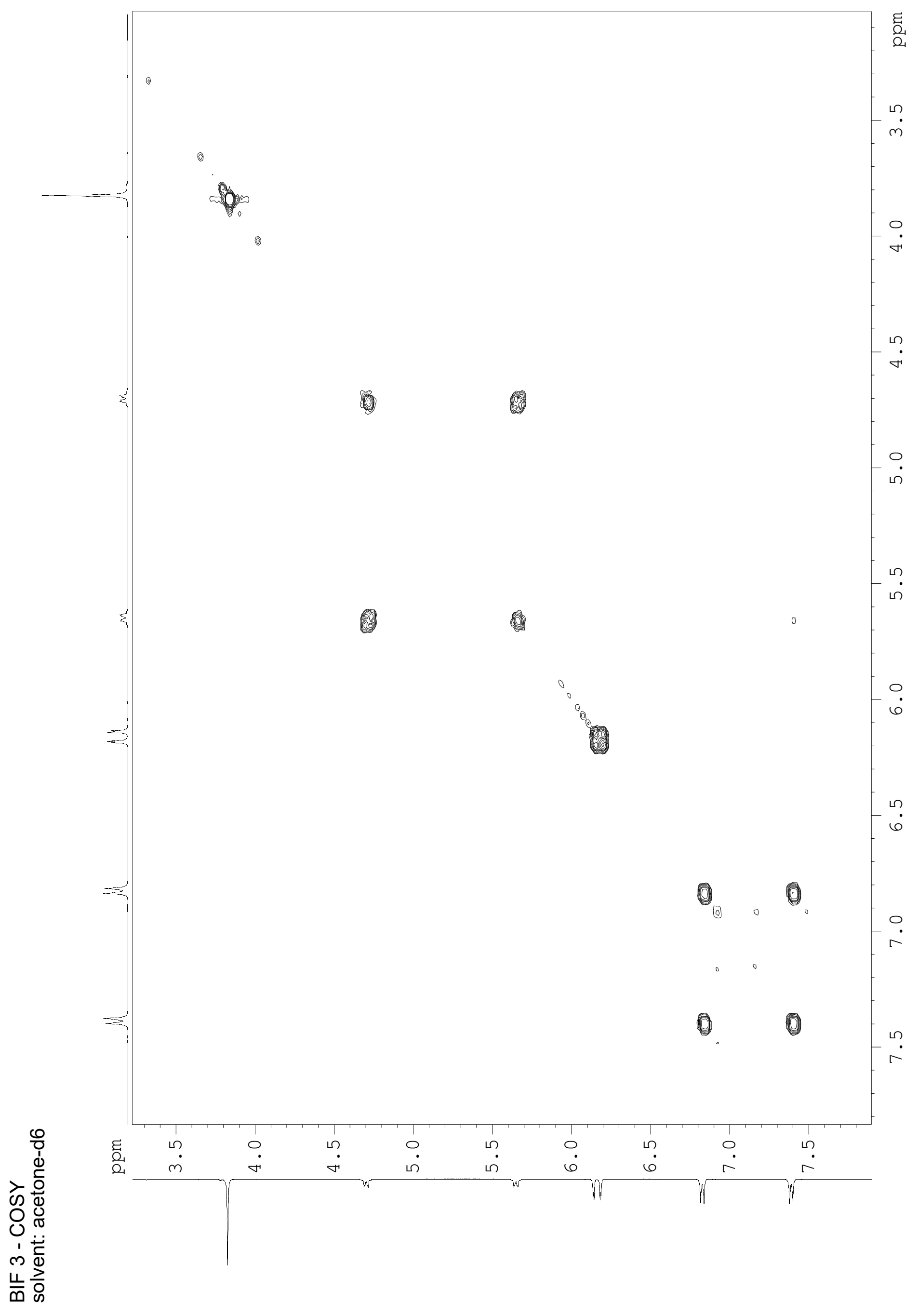




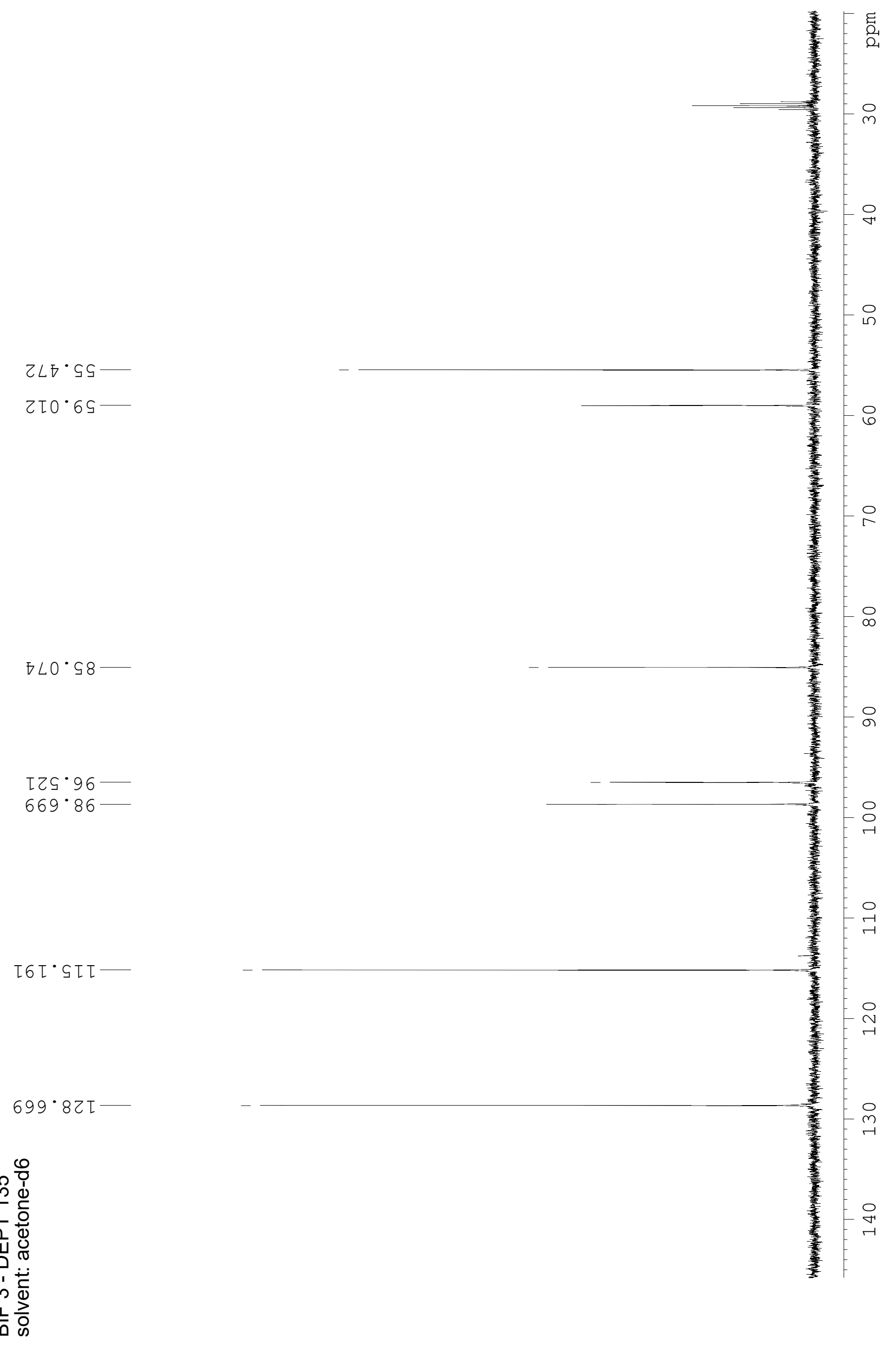




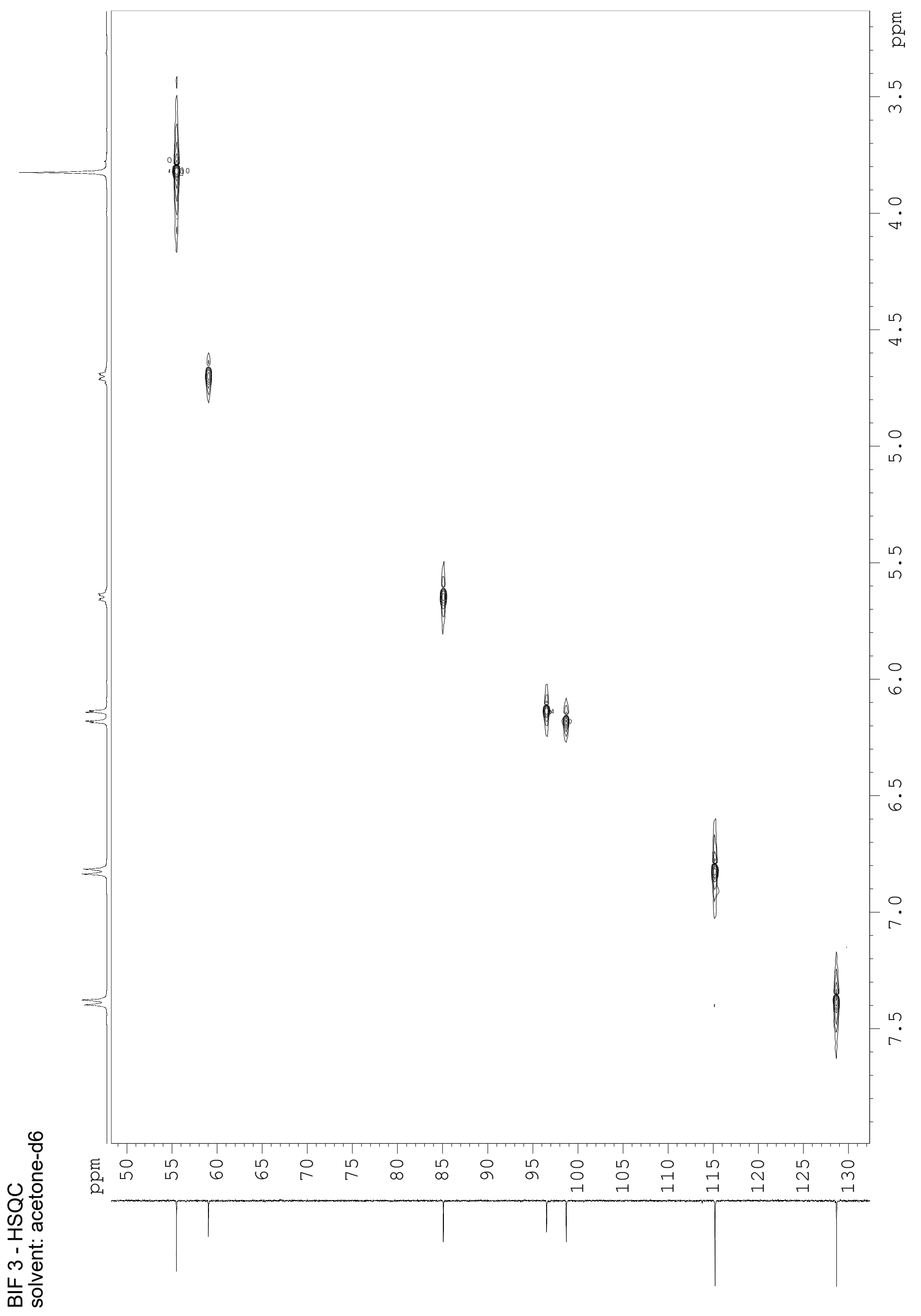




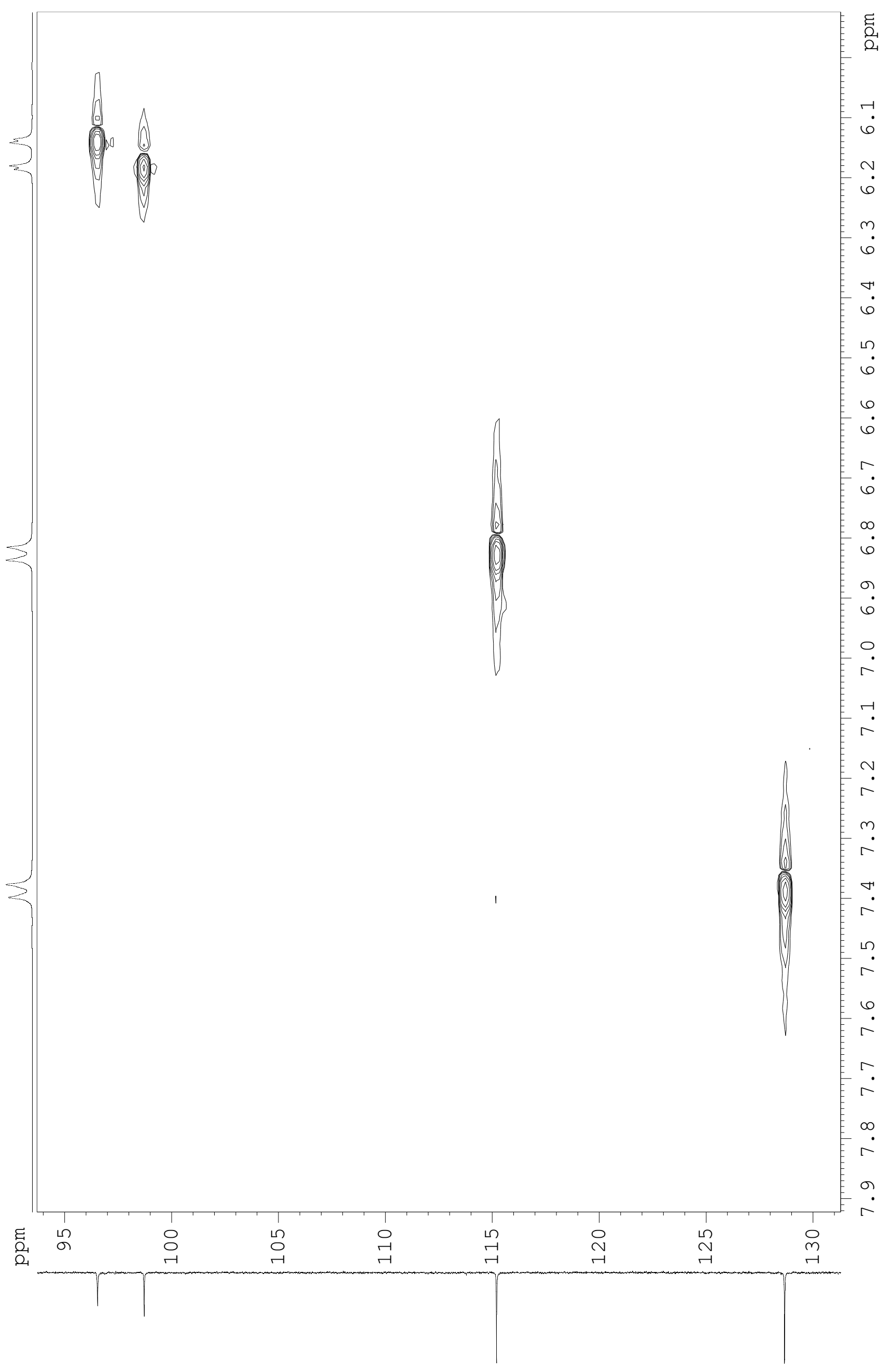




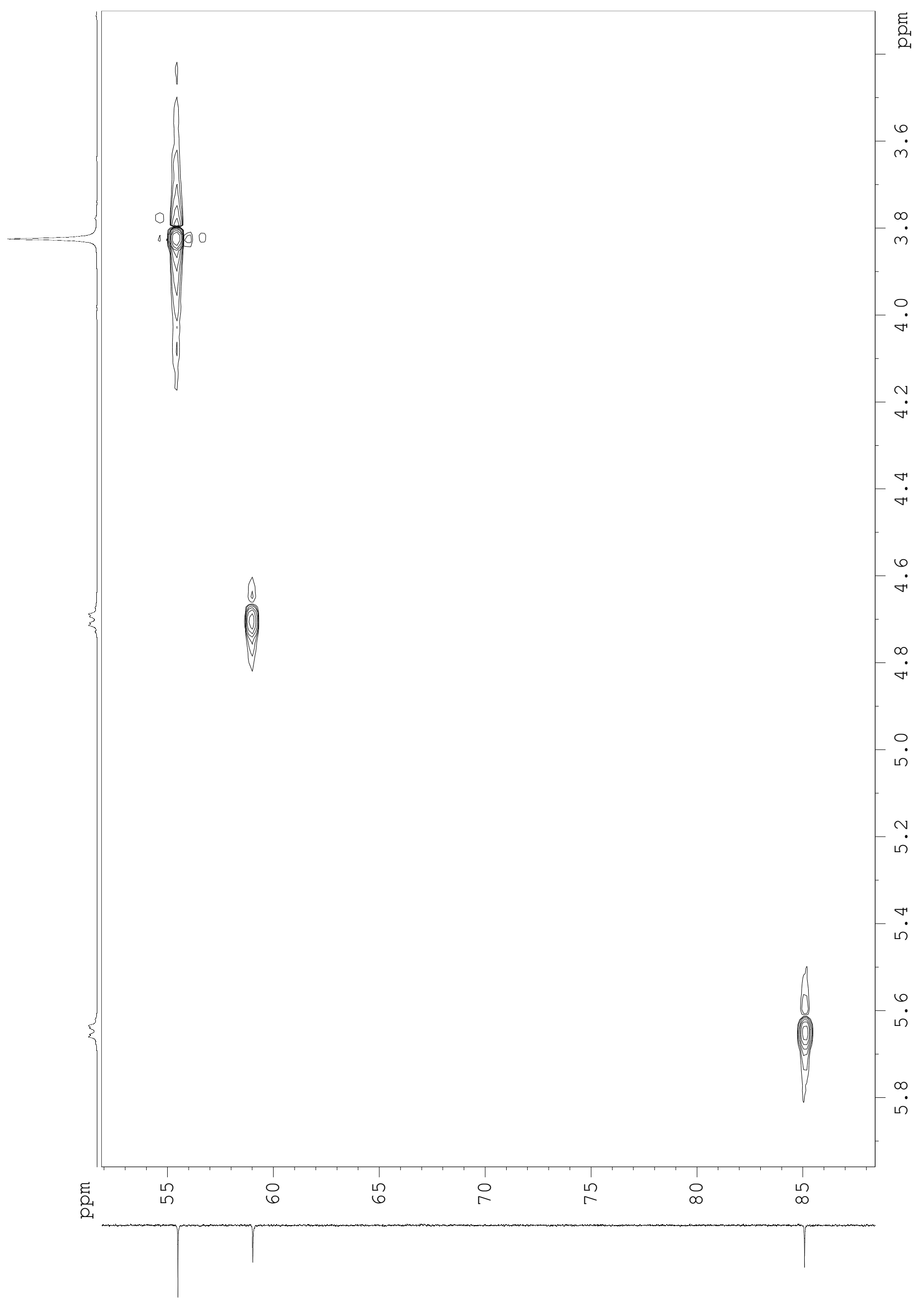




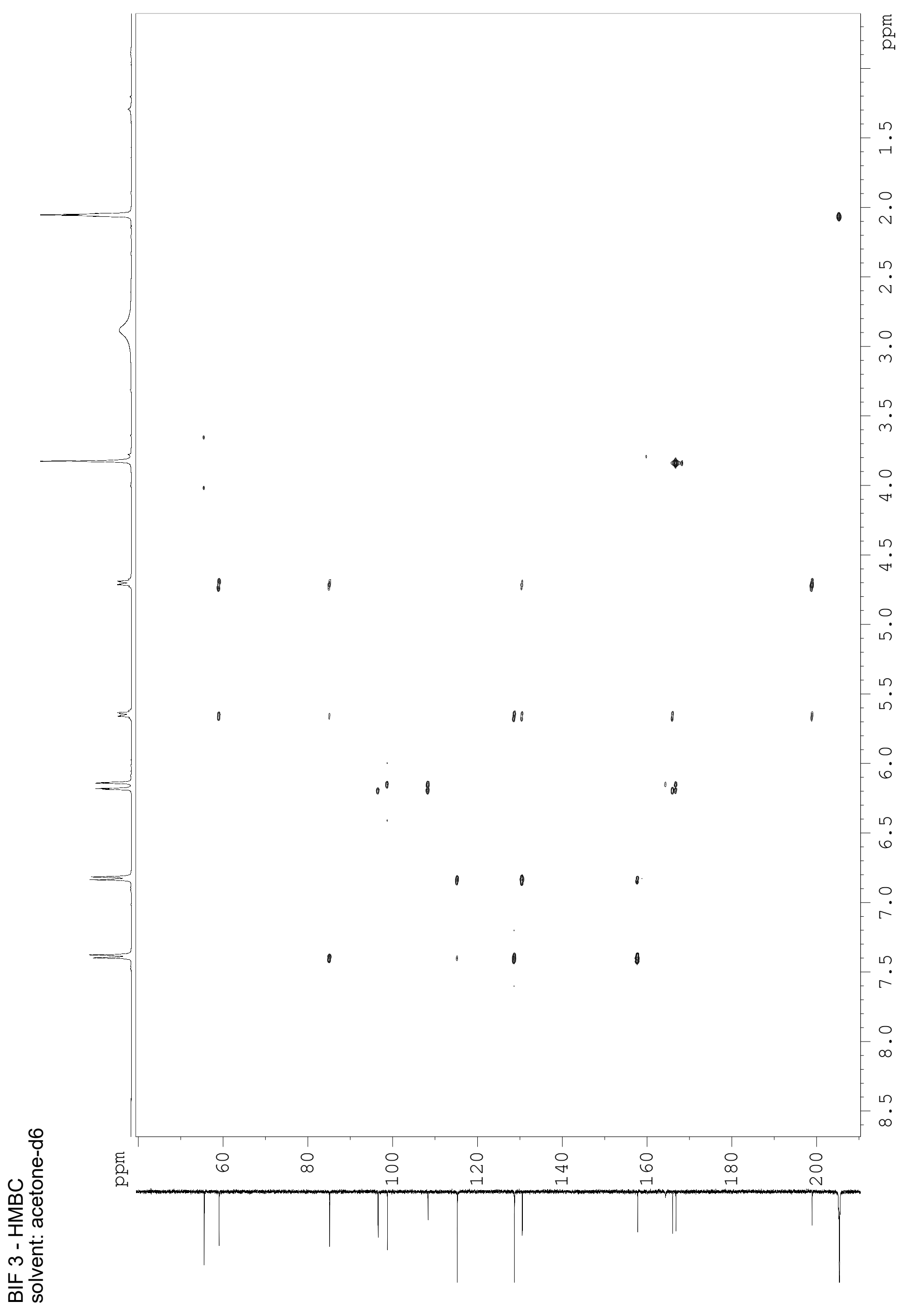




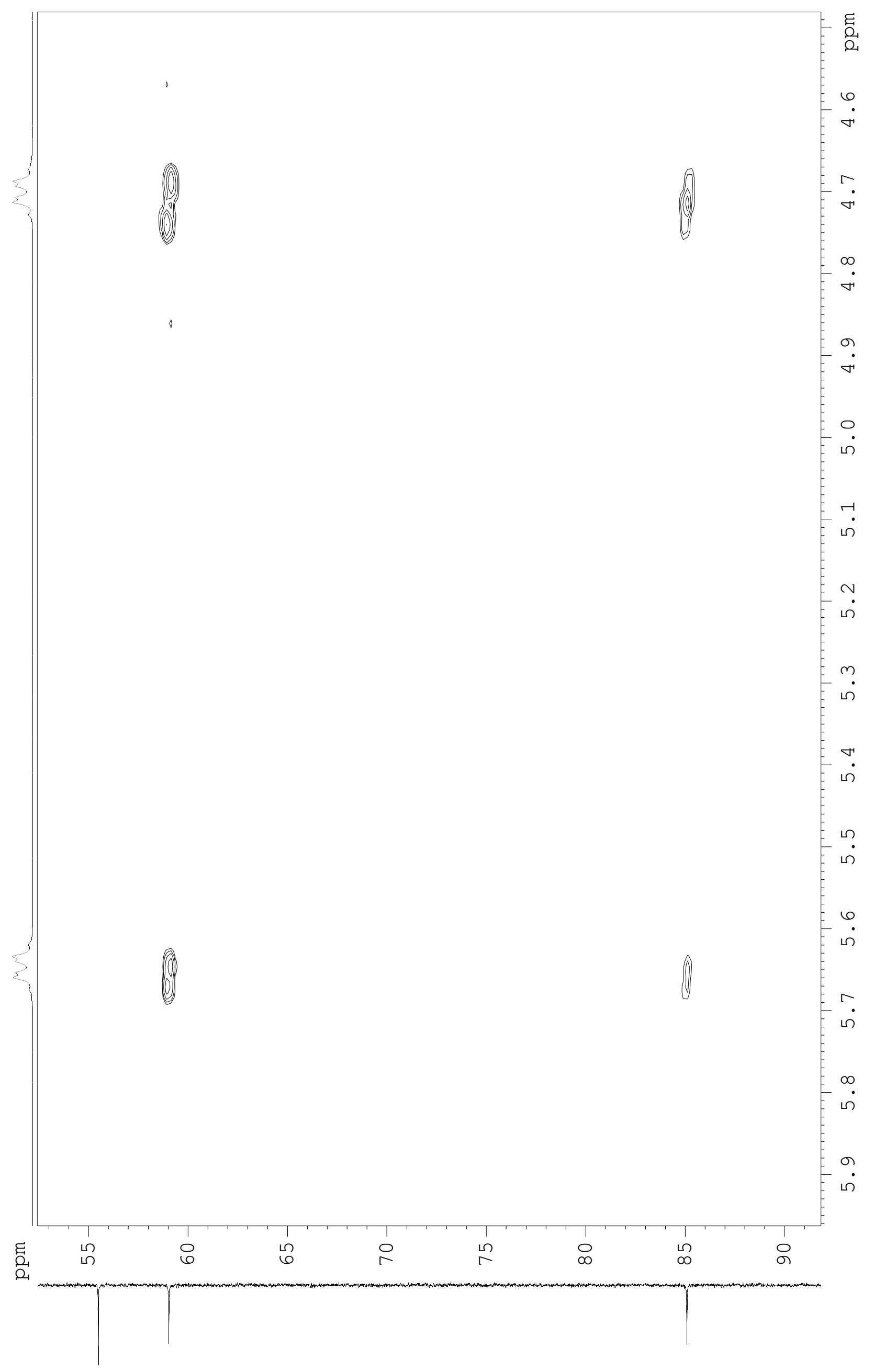




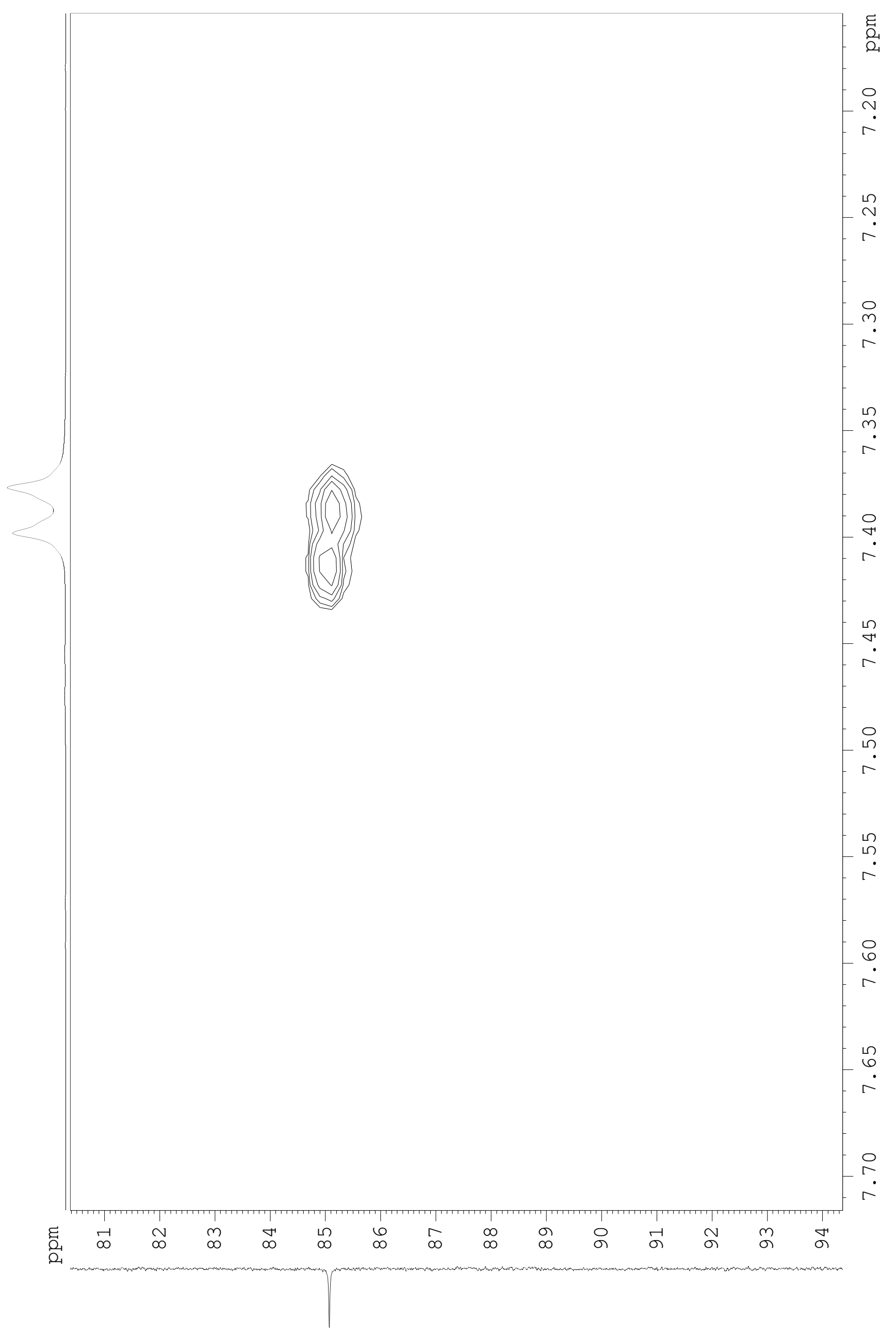




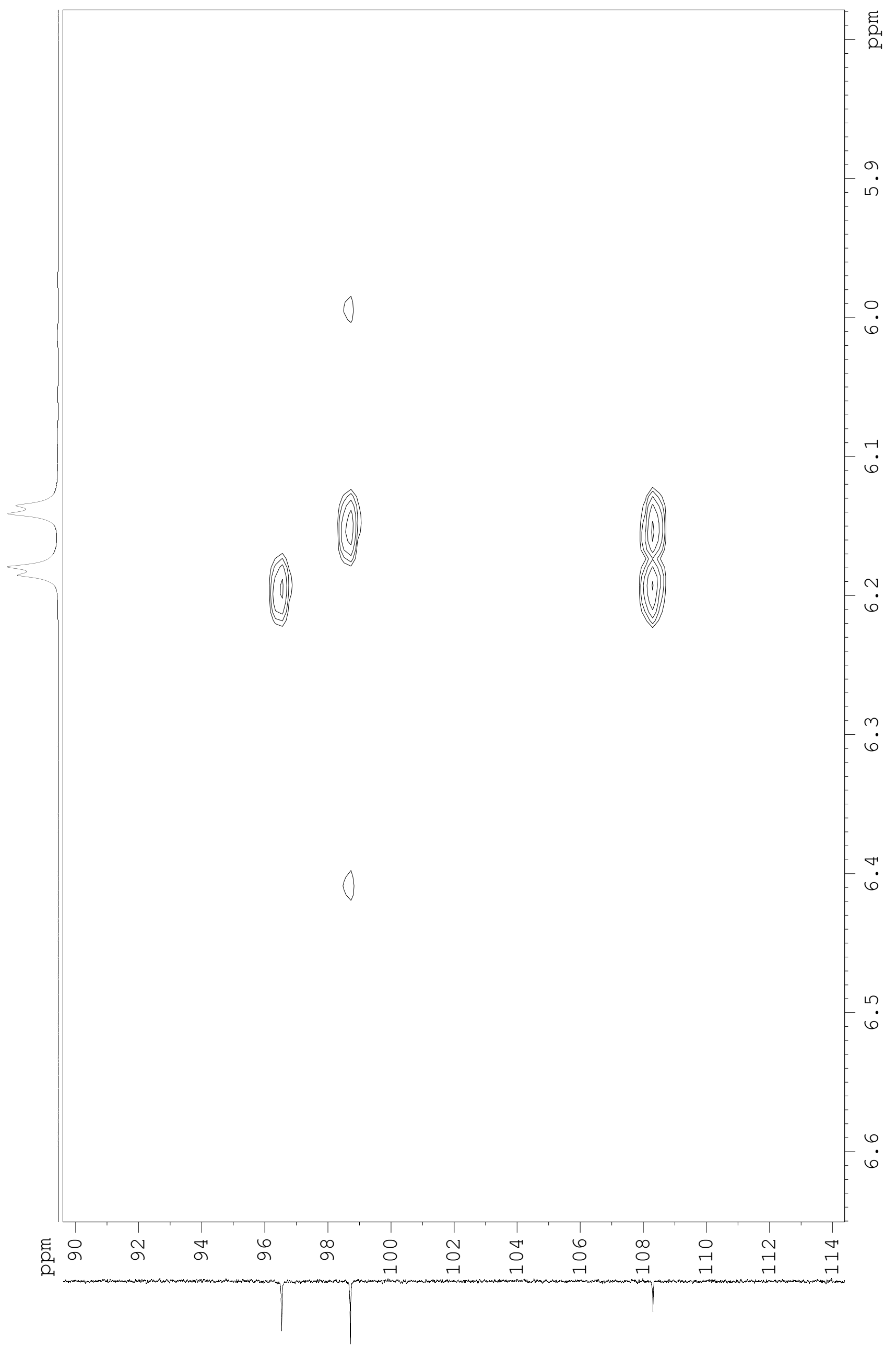




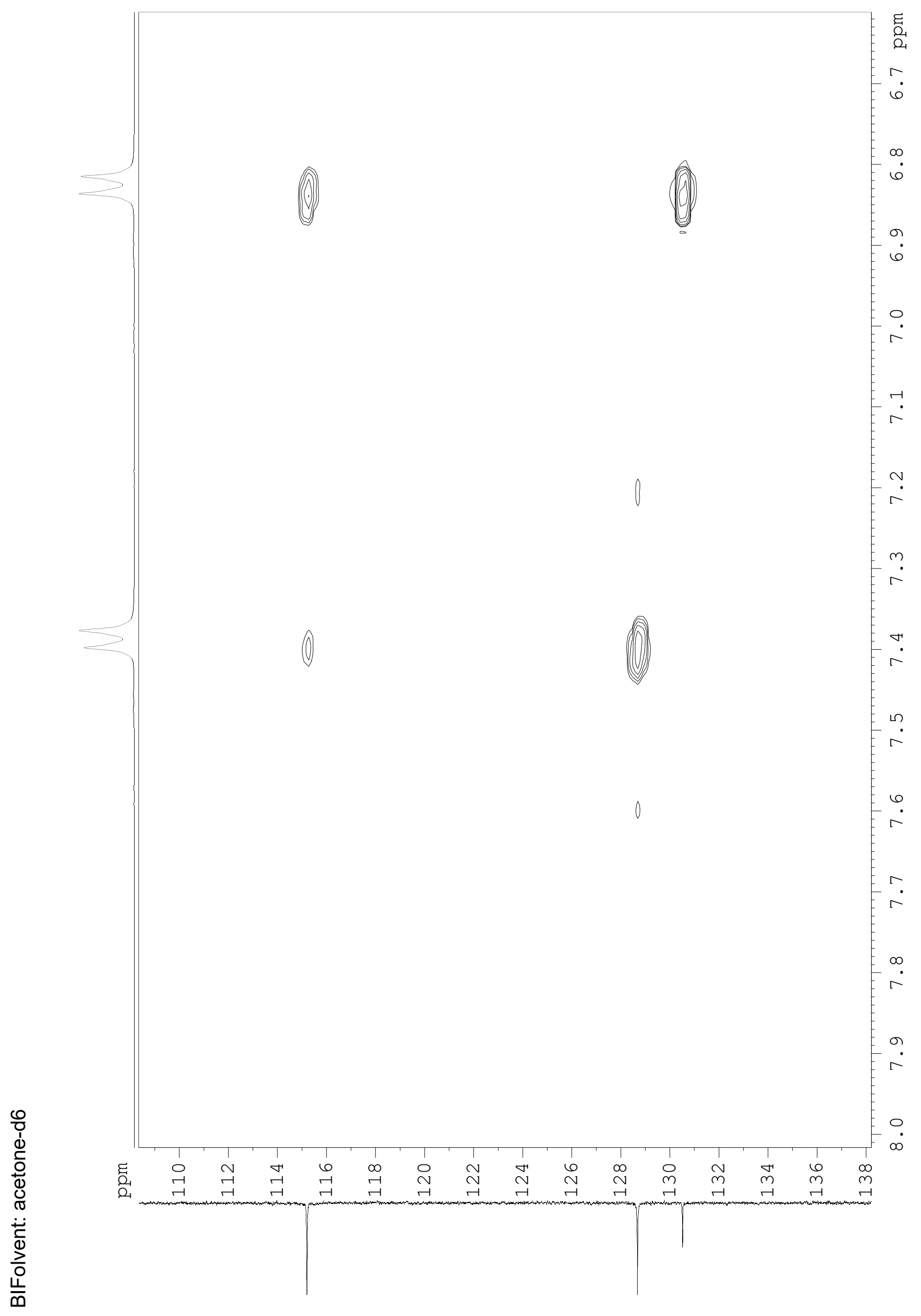




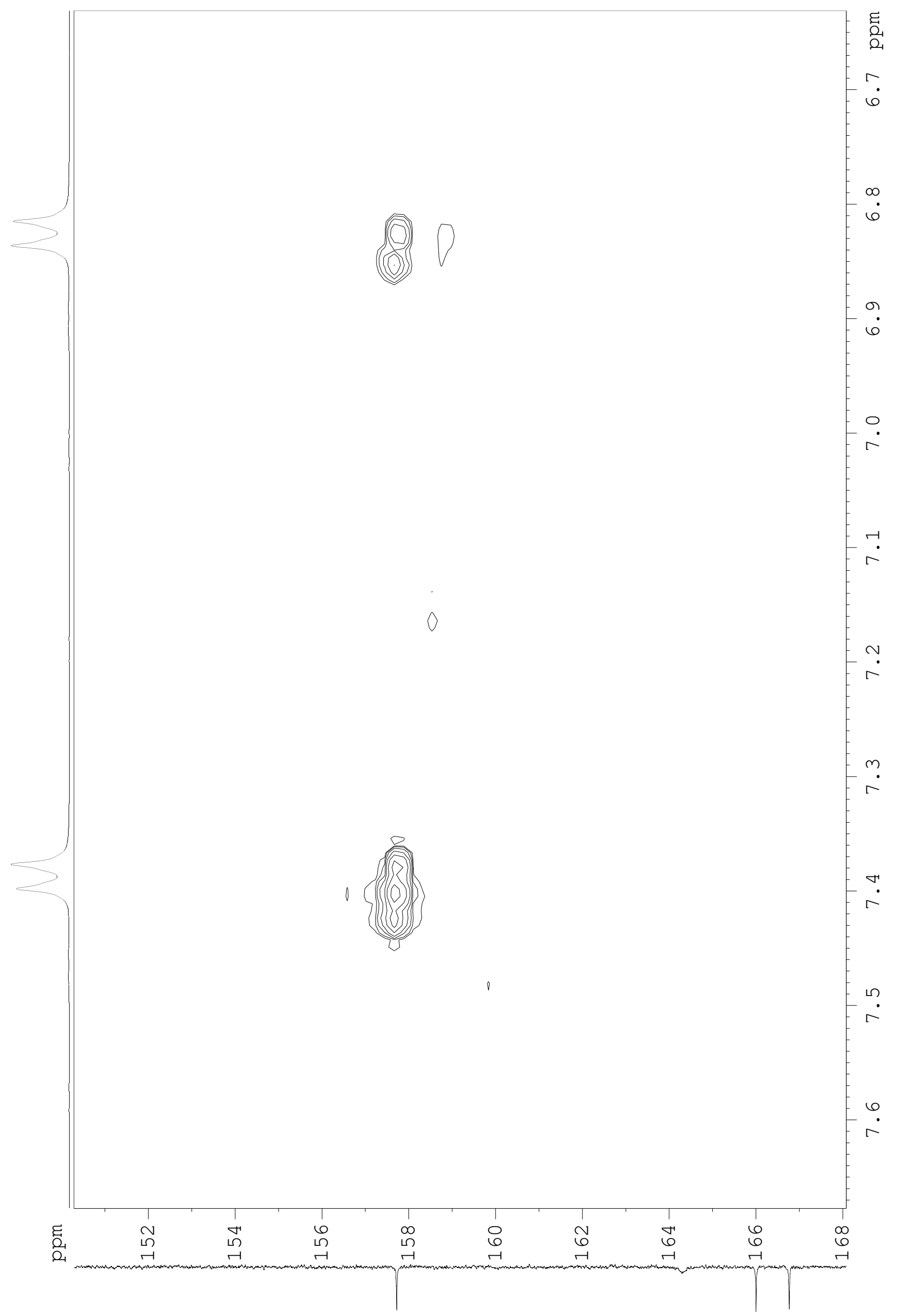




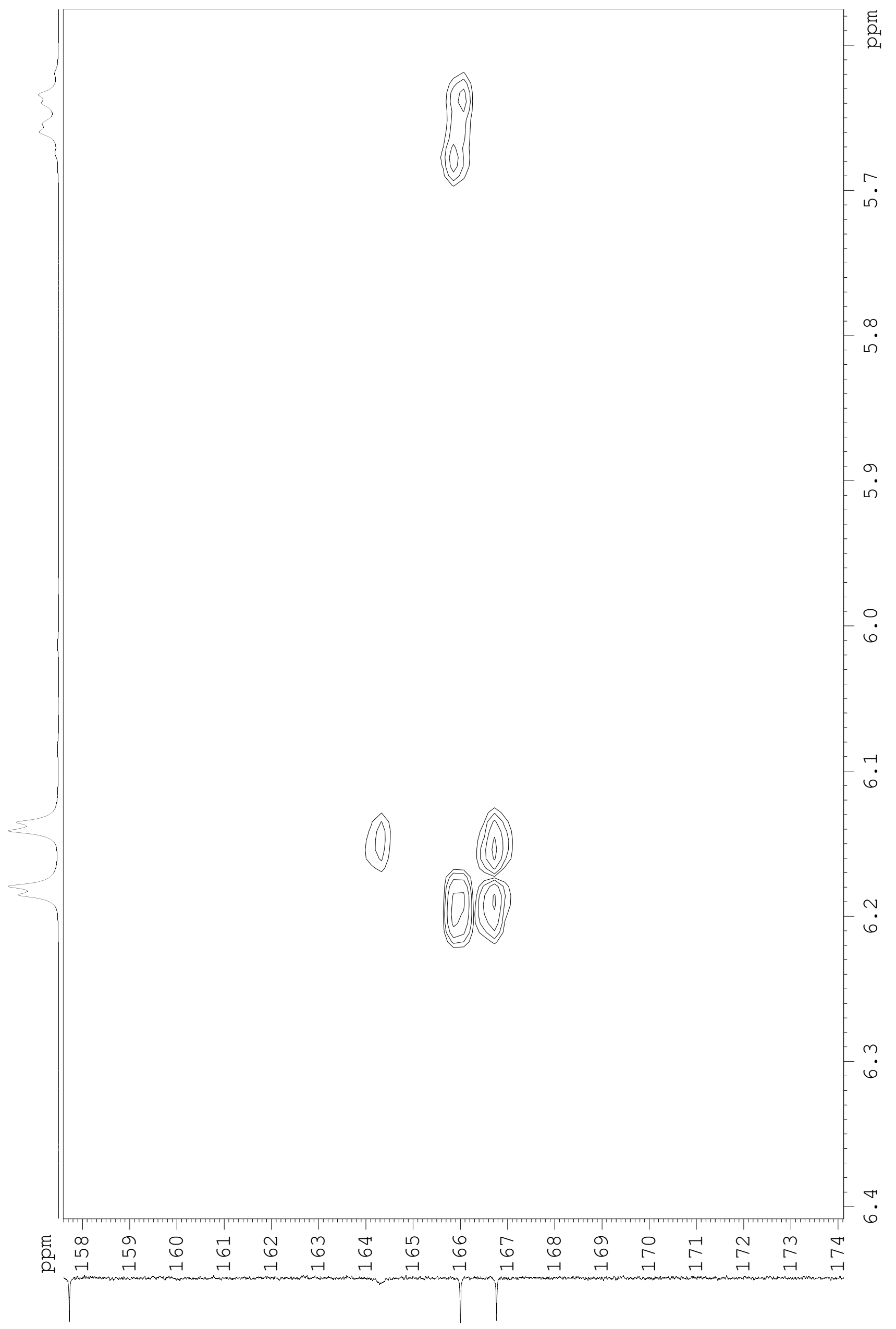




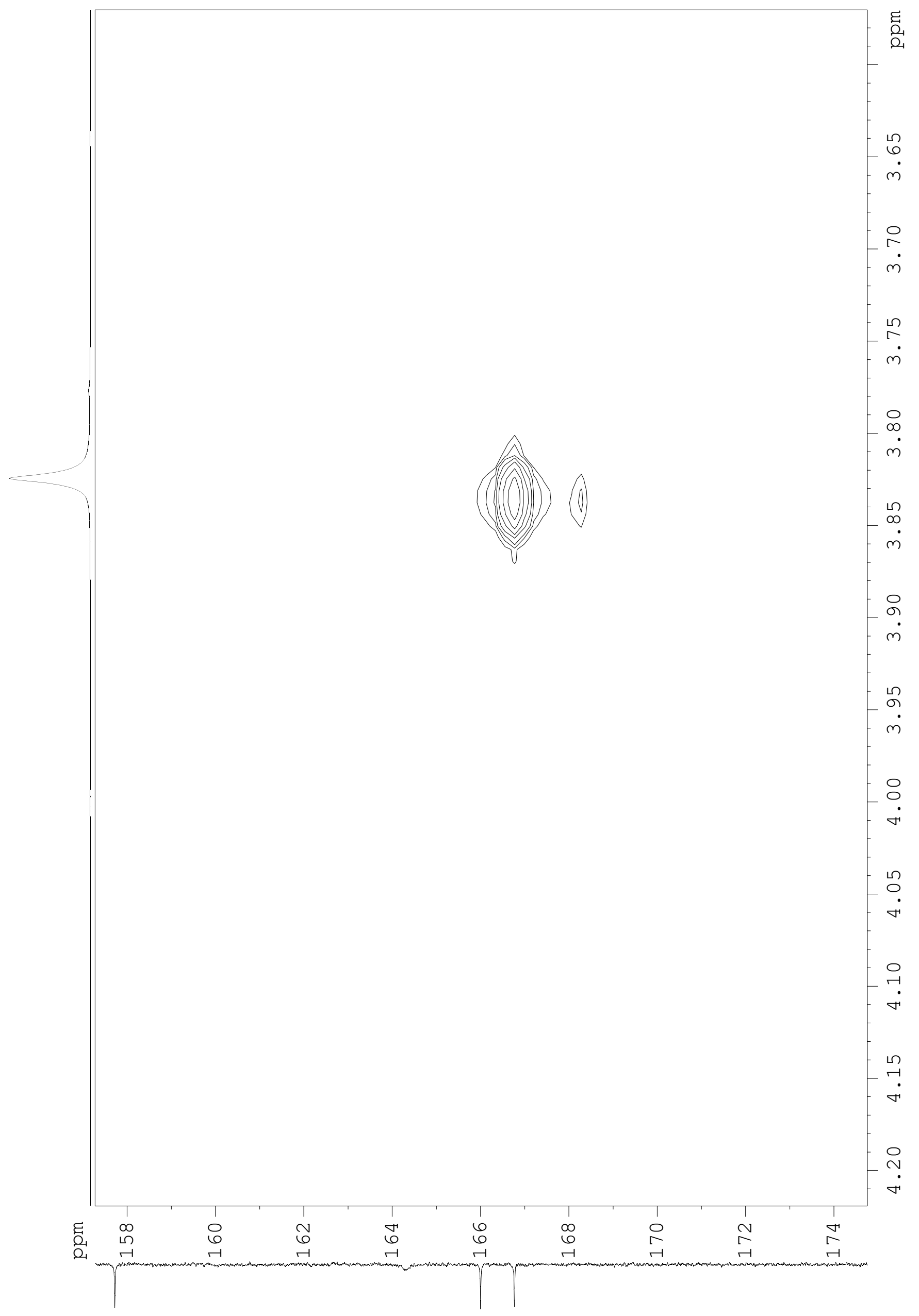




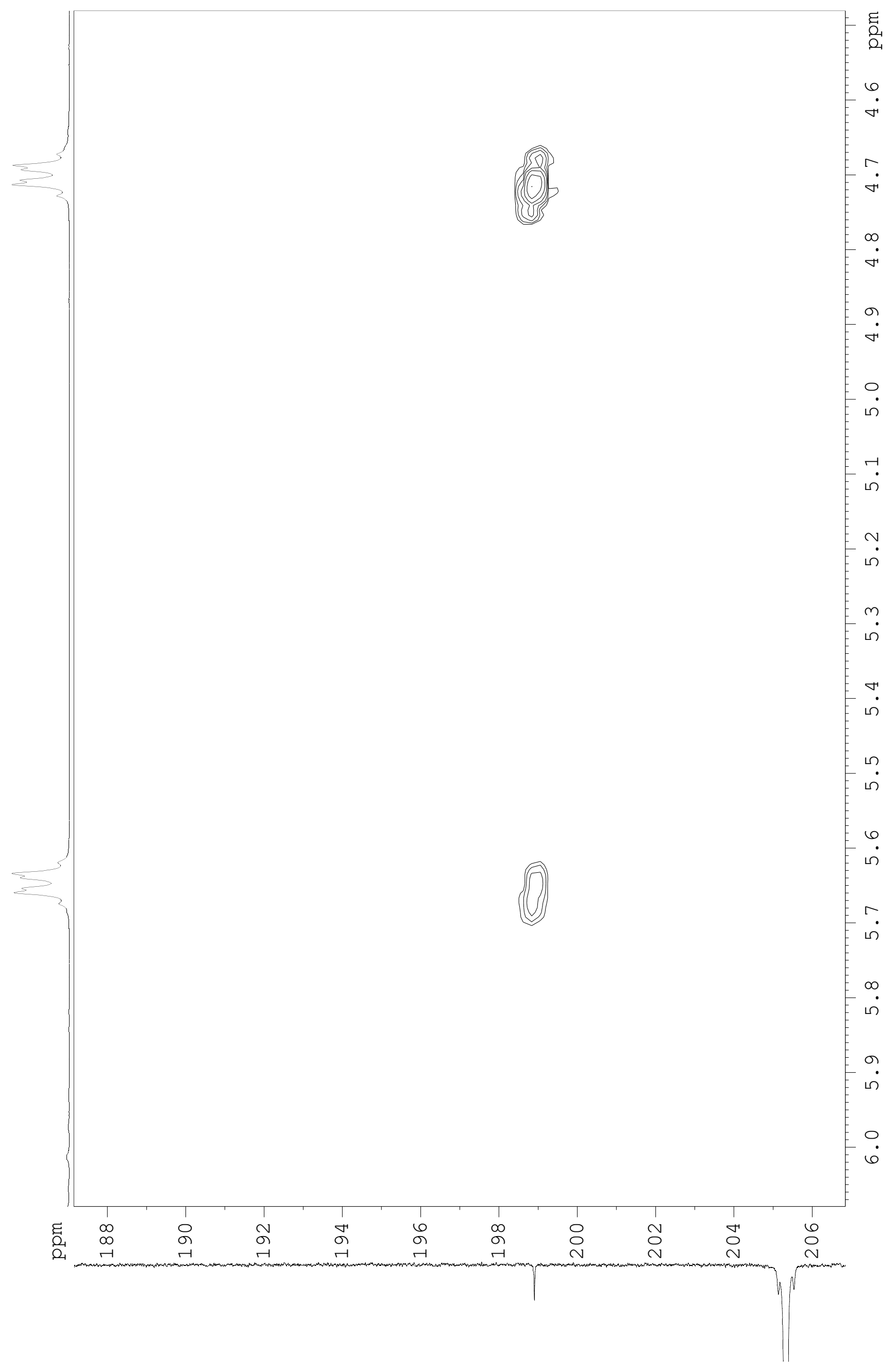




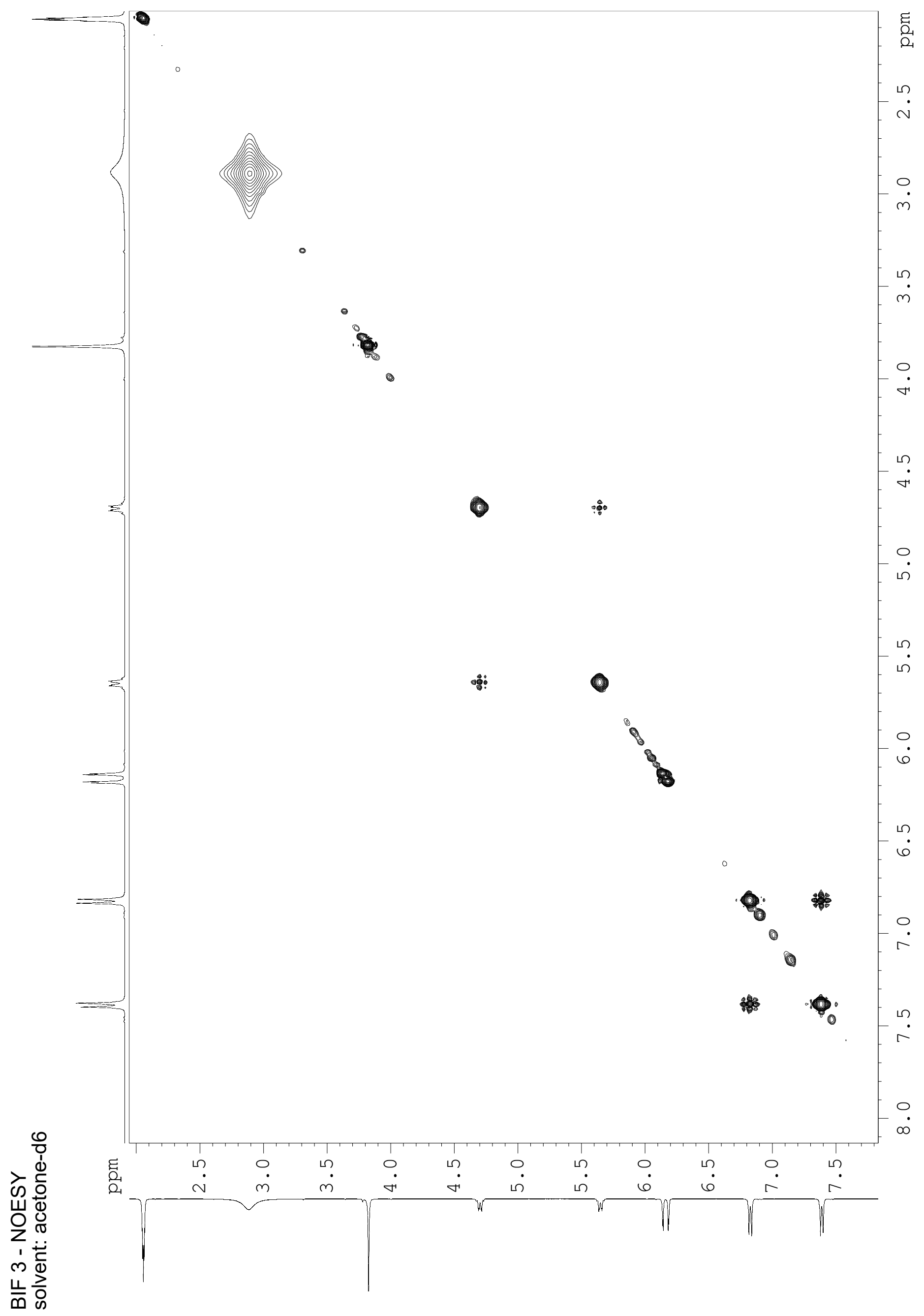


$โ 8 L \cdot \varepsilon-$

乙โ $\varepsilon^{\circ} \varepsilon$

$269^{\circ}$.

SIL.

$879^{\circ} 9$

$\varepsilon \angle 9^{\circ} \mathrm{S}$

$089^{\circ} \mathrm{s}$

SOL'S

$9 \varepsilon[\cdot 9$

$6 \varepsilon \tau \cdot 9$

$89 I \cdot 9$

$\varepsilon L T \cdot 9$

Zโ8.9

†E8.9

$\angle 68 \cdot 9$

$6[6 \cdot 9$

$\nabla L \varepsilon^{\circ}$

$\varsigma 6 \varepsilon^{\circ}$

$69 \sigma^{\circ}$

I6 $0^{\circ} L$
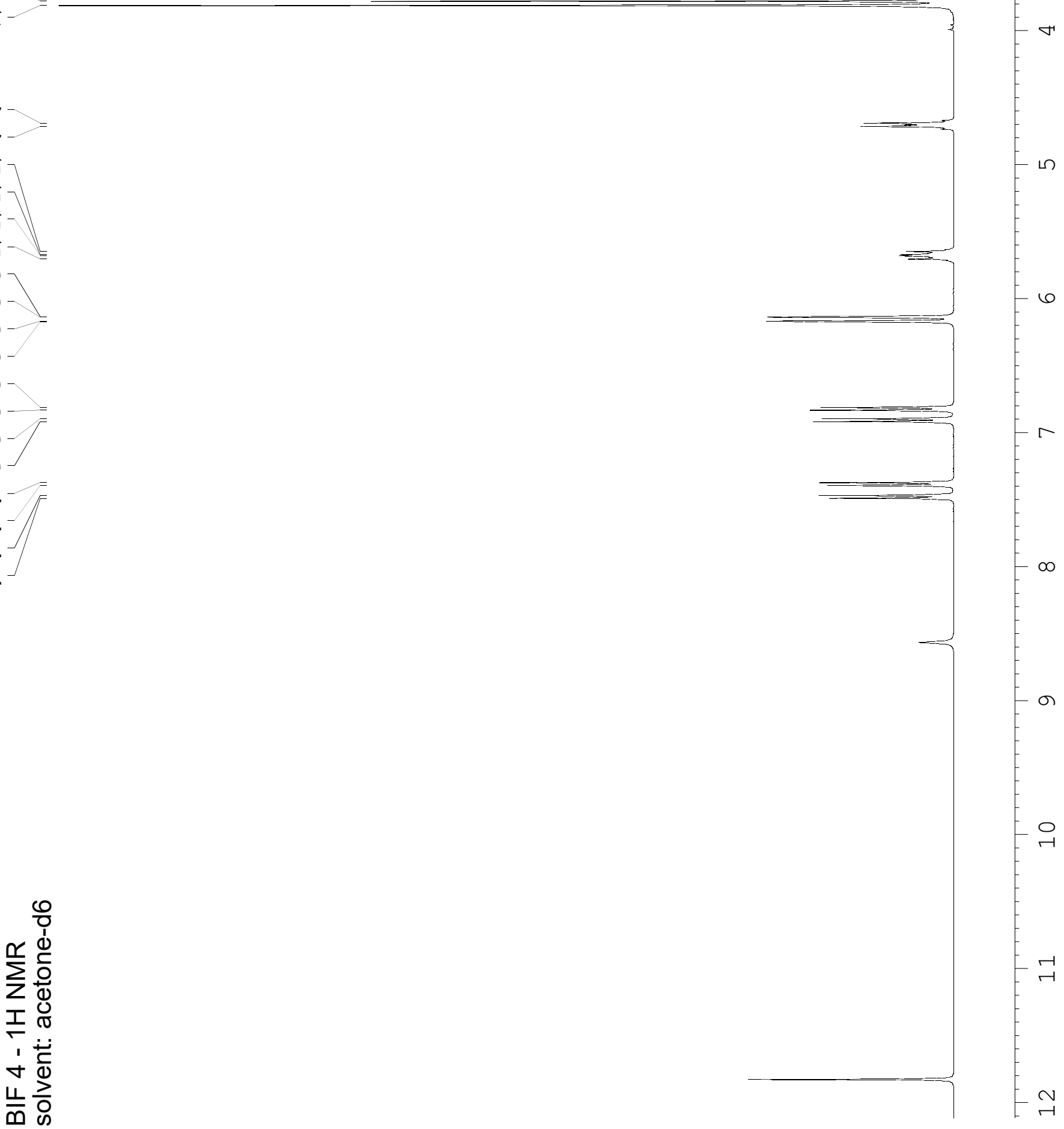
$โ 8 L \cdot \varepsilon-$

乙โ $8^{\circ} \varepsilon$

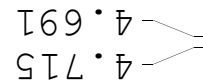

$879 \cdot 9-$

$\varepsilon \angle 9^{\circ} \mathrm{G}$

$089^{\circ} \mathrm{s}$

SOL'S-

$9 \varepsilon \tau \cdot 9$

$6 \varepsilon \tau \cdot 9$

$89 \tau \cdot 9$

$\varepsilon L I \cdot 9-$

乙โ8・9

ธह8 9

$\angle 68 \cdot 9$

$6\left[6^{\circ} 9\right.$

$\nabla L \varepsilon^{\cdot} L$

$96 \varepsilon^{\circ} L$

$69 \sigma^{\circ} L$

I6 $67^{\circ} L$ 
$9 \varepsilon \tau \cdot 9-$

$6 \varepsilon \tau \cdot 9-$

$89 I \cdot 9-$

$\varepsilon L \tau \cdot 9-$

乙โ8・9-

$\sqsubset 8^{\bullet} 9$

$468 \cdot 9-$

$6[6 \cdot 9$

$\nabla L \varepsilon^{\bullet} L-$

$\varsigma 6 \varepsilon^{\circ} L-$

$697^{\circ} L-$

I6 $7^{\circ} L$

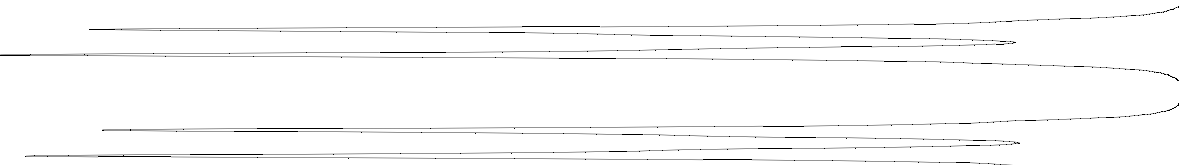

6

a

6

?

r

-

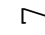

N 
I69.

SIL・五

हू

$=r$

$\infty$

$\nabla$

-

†

$\stackrel{0}{\circ}$

เก

ㄷ.

$=\frac{N}{}$

$879 \cdot 9-$

$\varepsilon \angle 9^{\circ} S^{-}$

$089^{\circ} S^{-}$

SOL. 9 
$โ 8 L \cdot \varepsilon$

$\tau โ 8^{\circ} \varepsilon$ 


$$
7
$$


$\sum$ 
ZहS.6ZI$8 \tau 9 \cdot 6 Z I-$ 
$L L Z \cdot 60 I-$ 
$208 \cdot 98-$

$696^{\circ} 98$

$\angle Z G^{*} \angle 6$

$\varepsilon \tau L \cdot 66-$

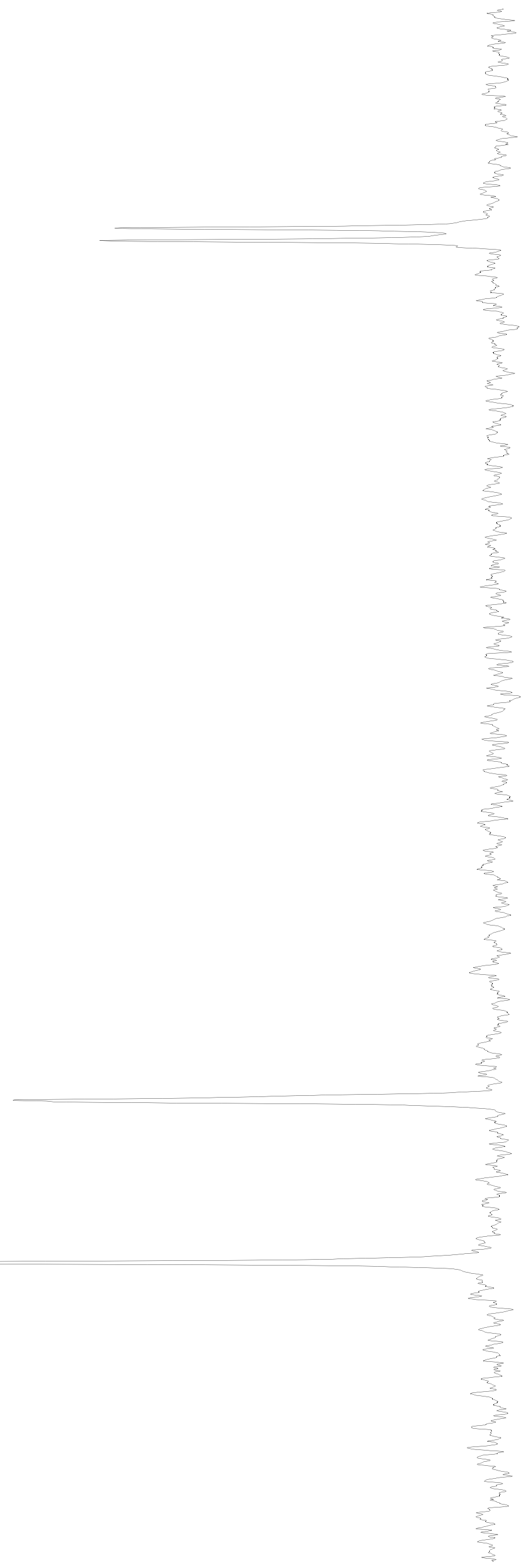


$956 \cdot 69-$ $286^{\circ} 69$ 
$96 \varepsilon \cdot 9 \varsigma$

L8I.9S

$999 \cdot 69$

$S \varepsilon L \cdot 6 S$

$S L L \cdot 6 S-$

Ђง $9 \cdot 98$

$\varepsilon 乙 L \cdot S 8$

$9 L Z \cdot \angle 6$

$\varepsilon 0 \varepsilon^{\bullet} \angle \sigma$

$\varepsilon 9 \sigma^{\circ} 66^{-}$

$L \varepsilon \sigma^{\circ} S I T-$

$98 Z \cdot 62 \tau$

ZLE・6ZI

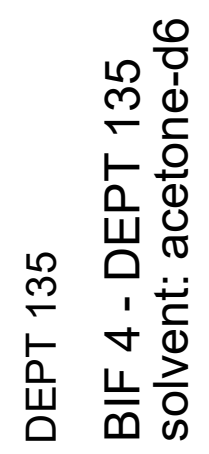


9 Z与・五I

$L \varepsilon \sigma^{\circ} \mathrm{ST \tau}$

$98 Z \cdot 6 Z I-$

ZLE'6ZT 
Ђ马S S 8

$\varepsilon 乙 L \cdot \hookrightarrow 8$ 
$96 \varepsilon \cdot \varsigma \hookrightarrow-$

$48 I \cdot 99$

$999 \cdot 69-$

$S \varepsilon L \cdot 6 \varsigma$

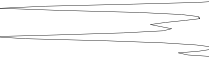




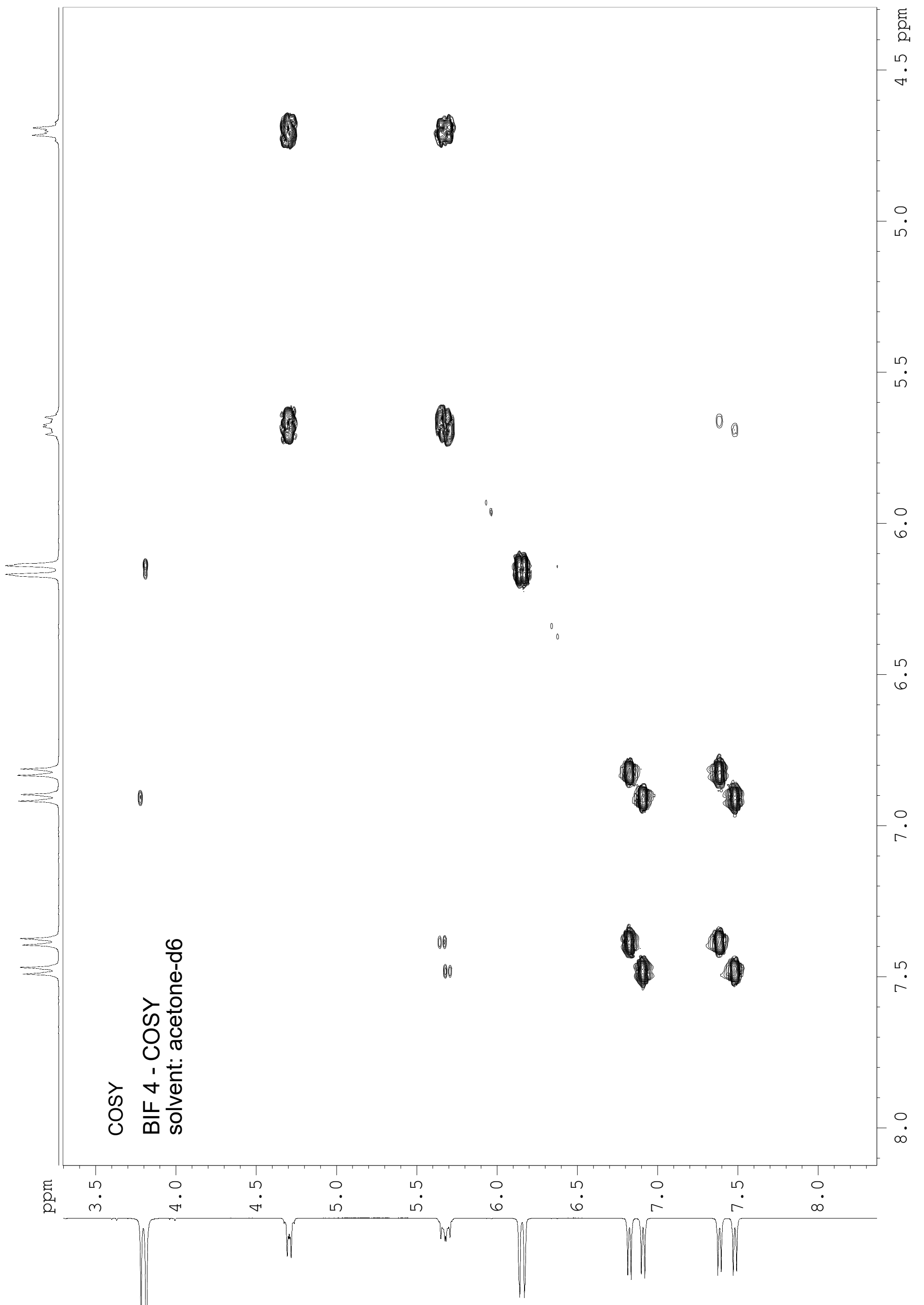




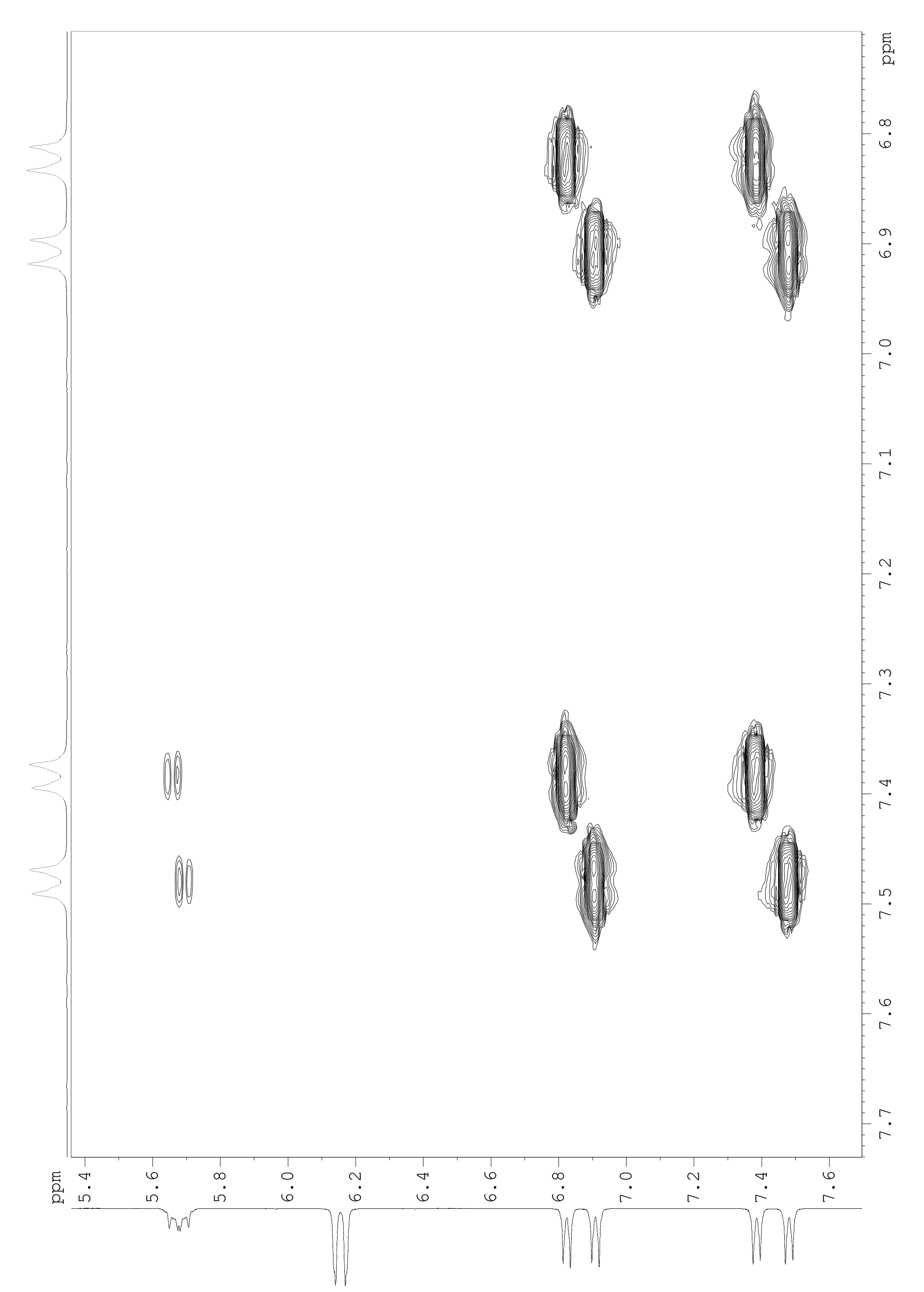




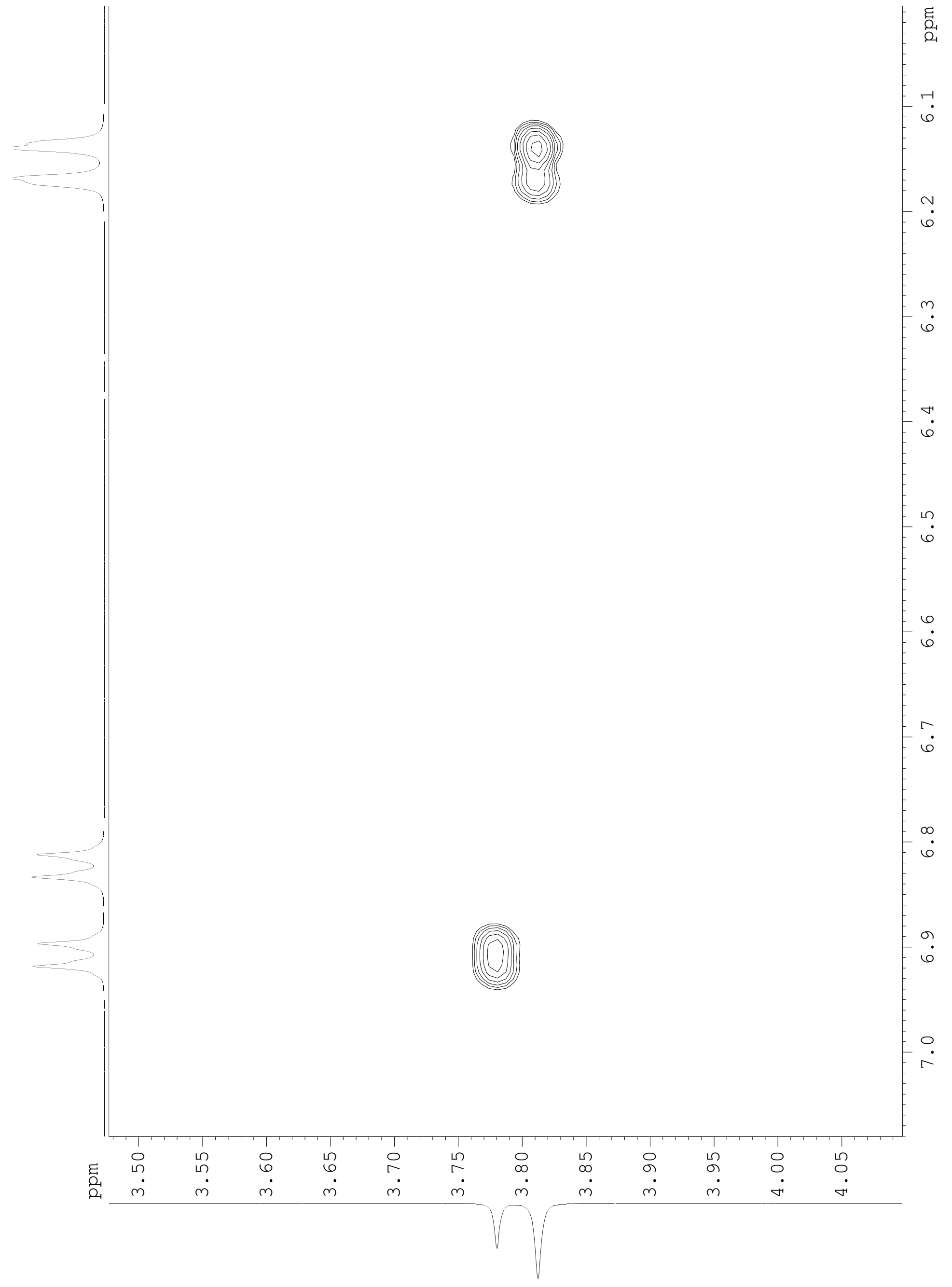




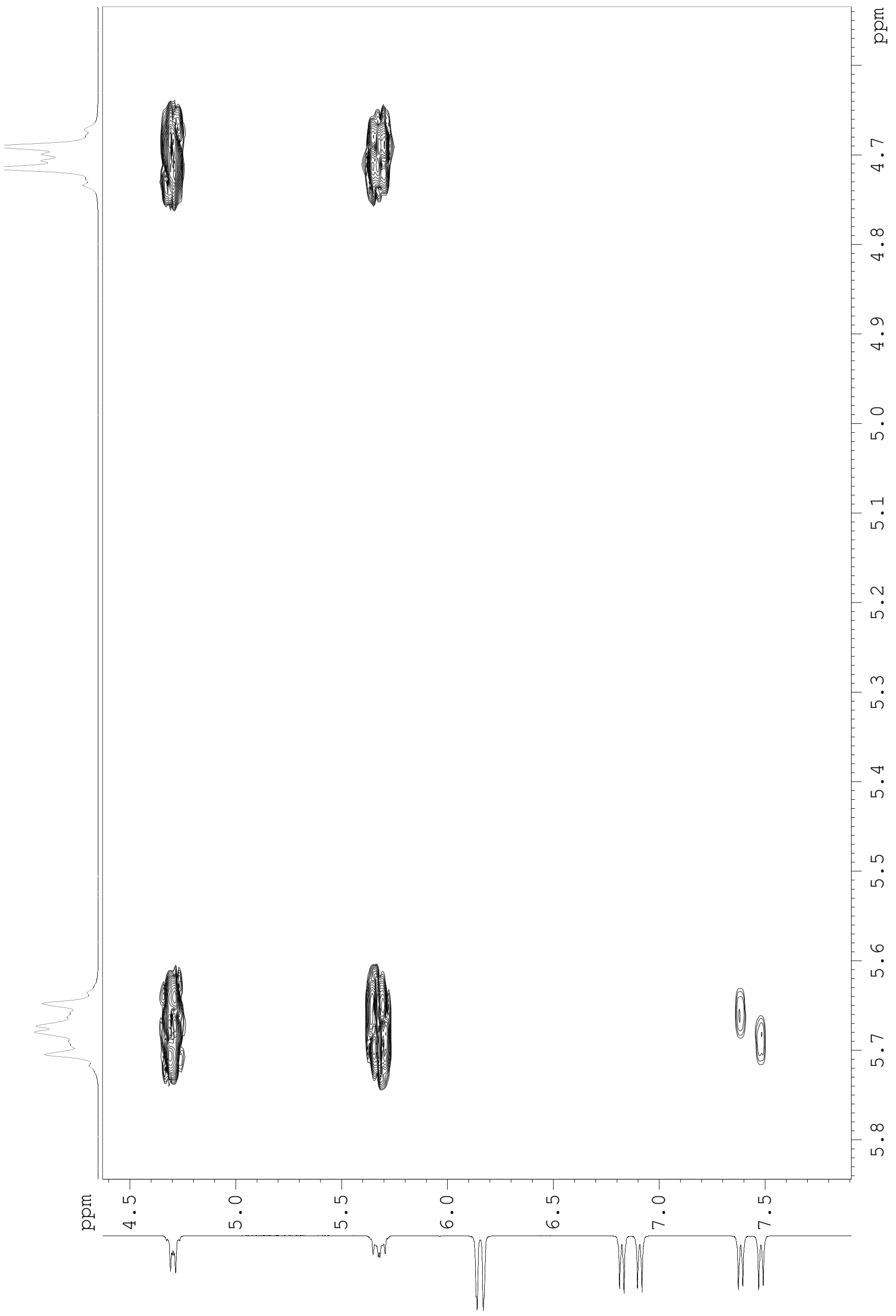




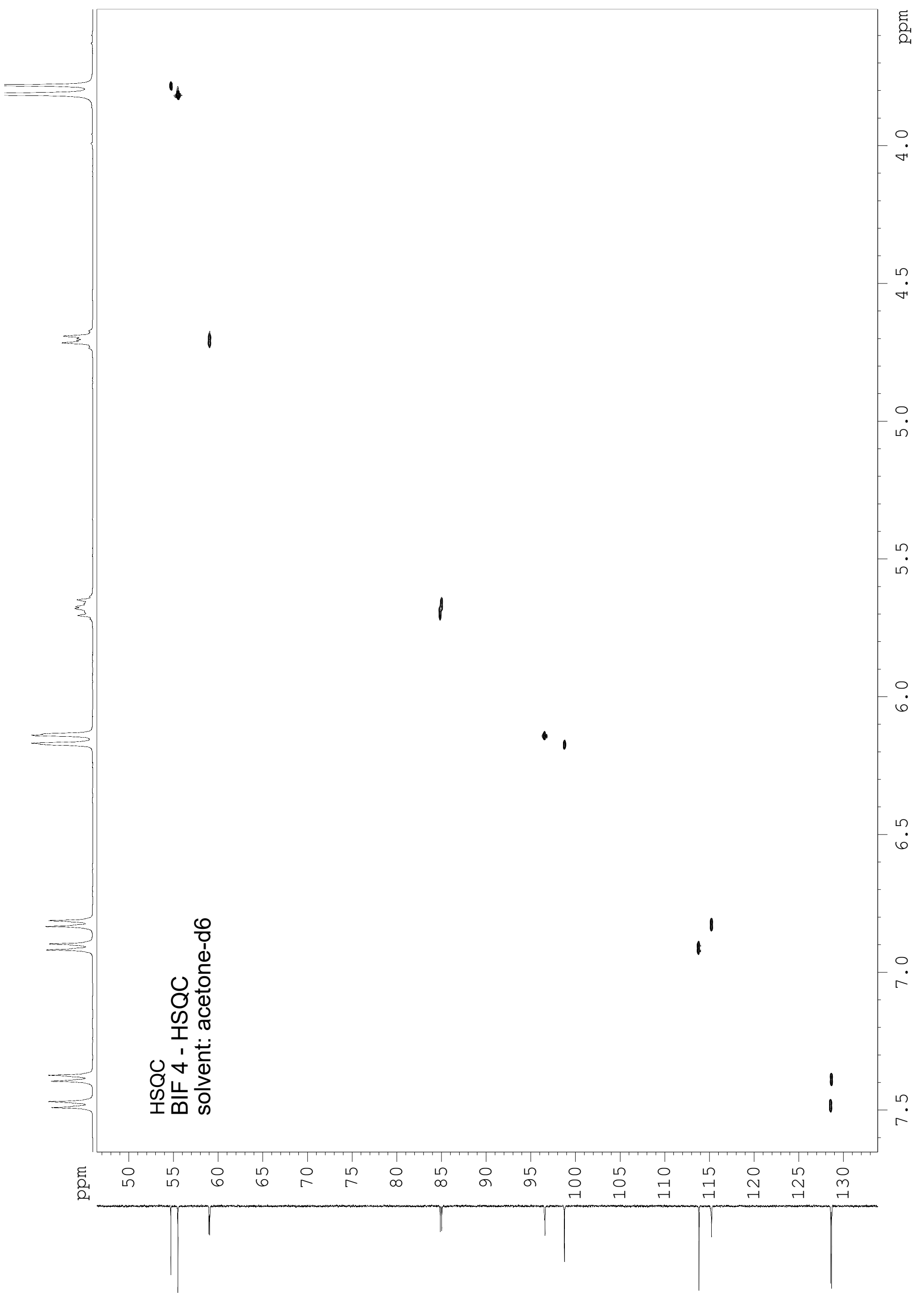


(2)

(1) 


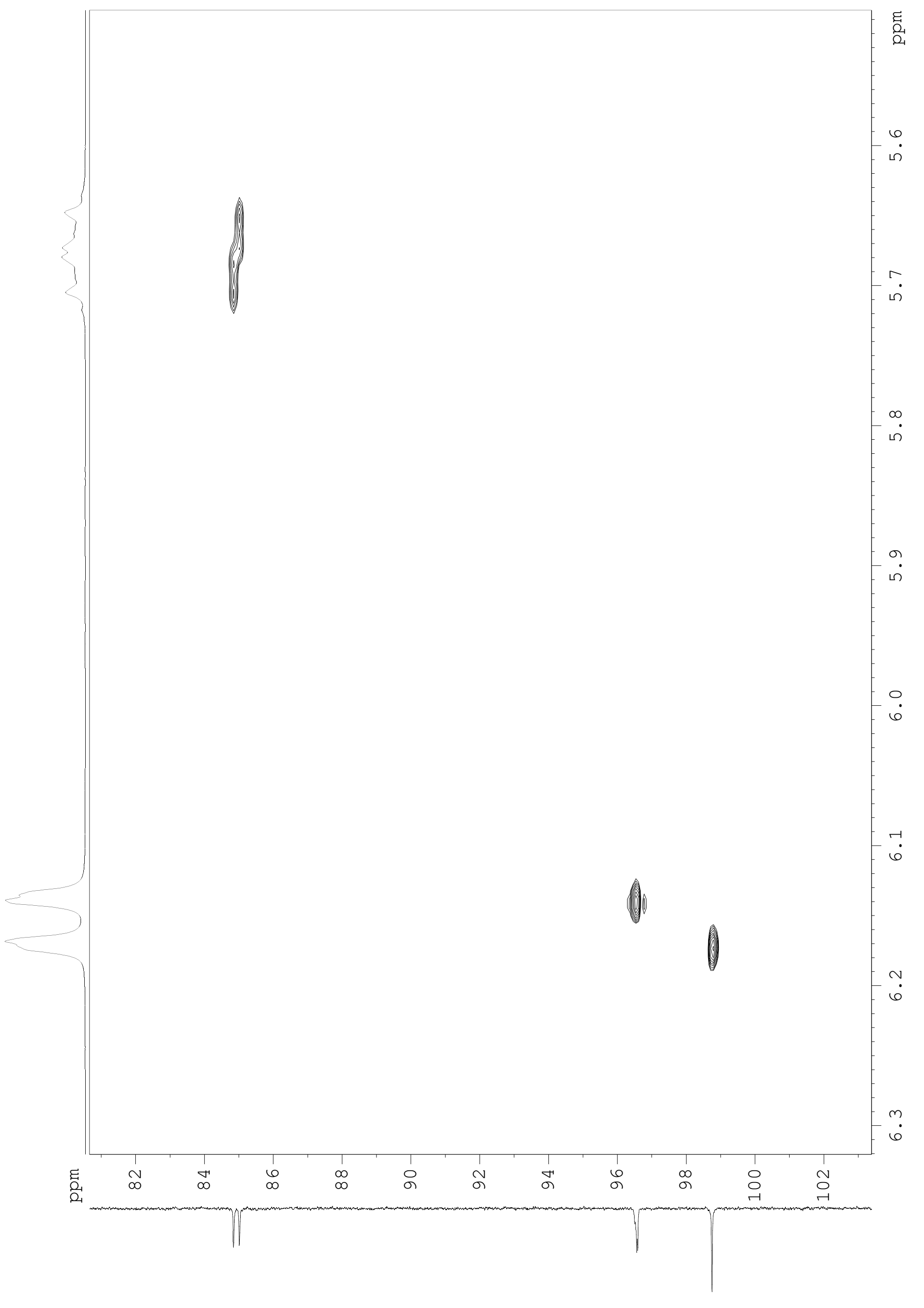




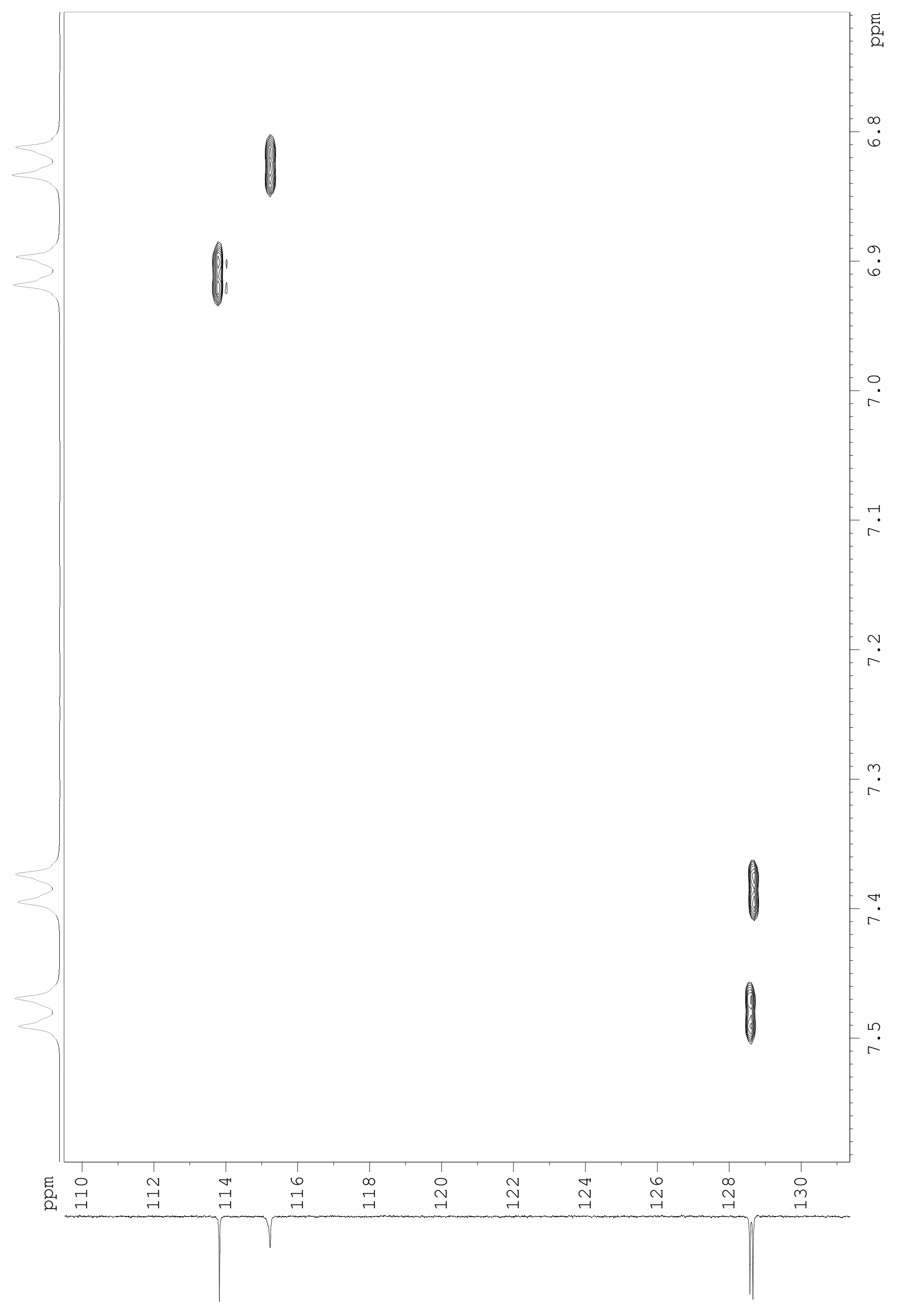




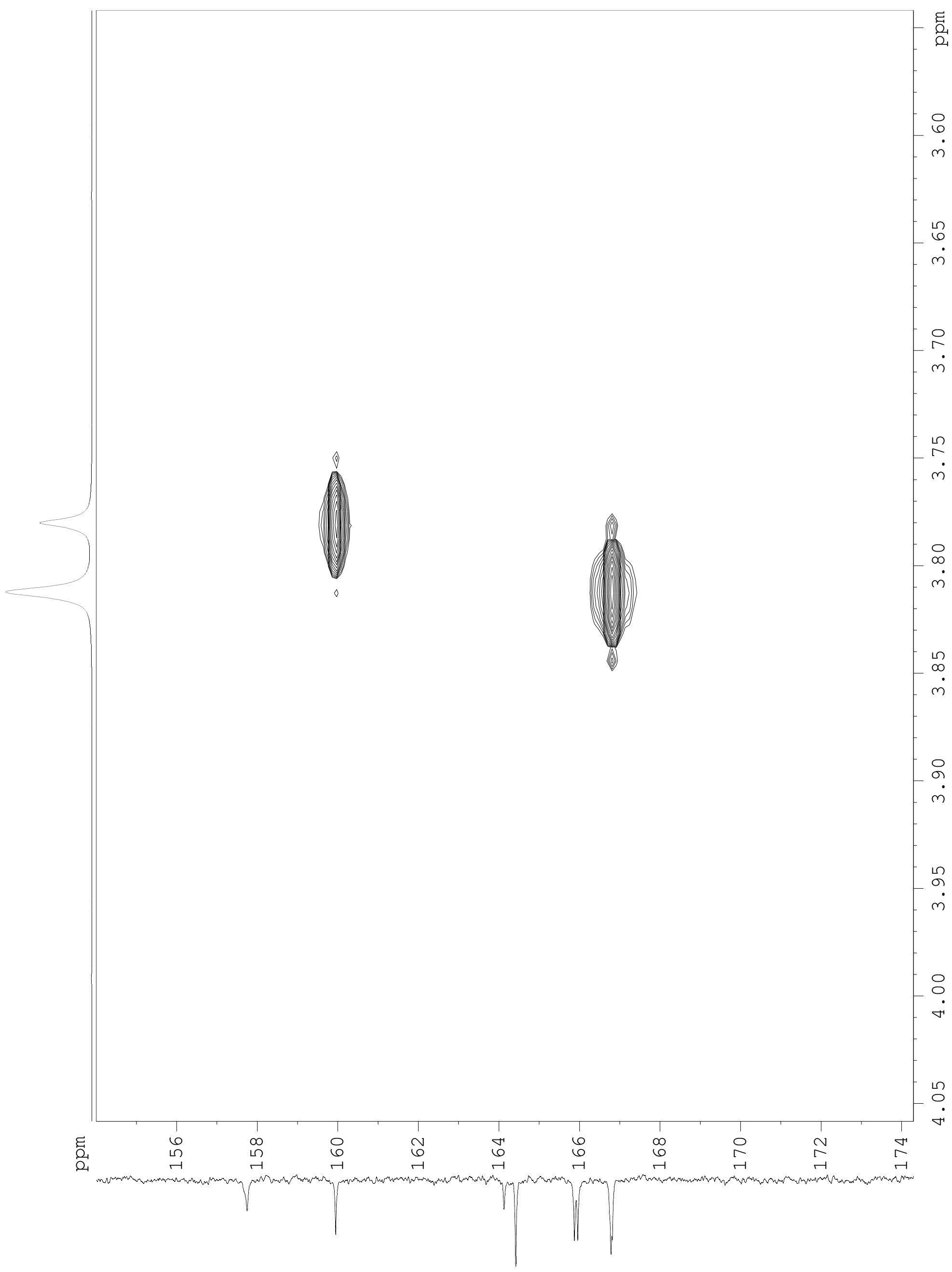




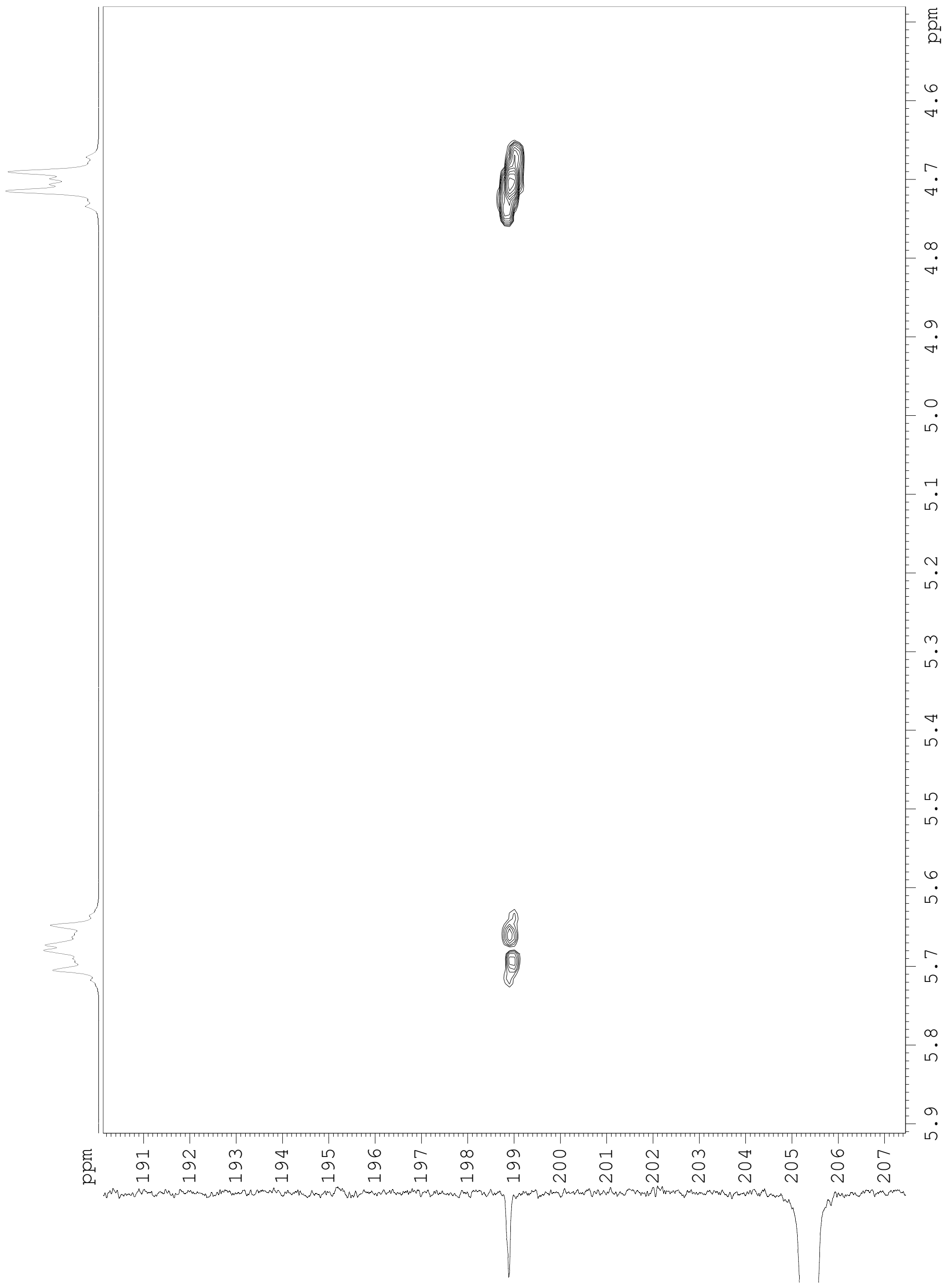




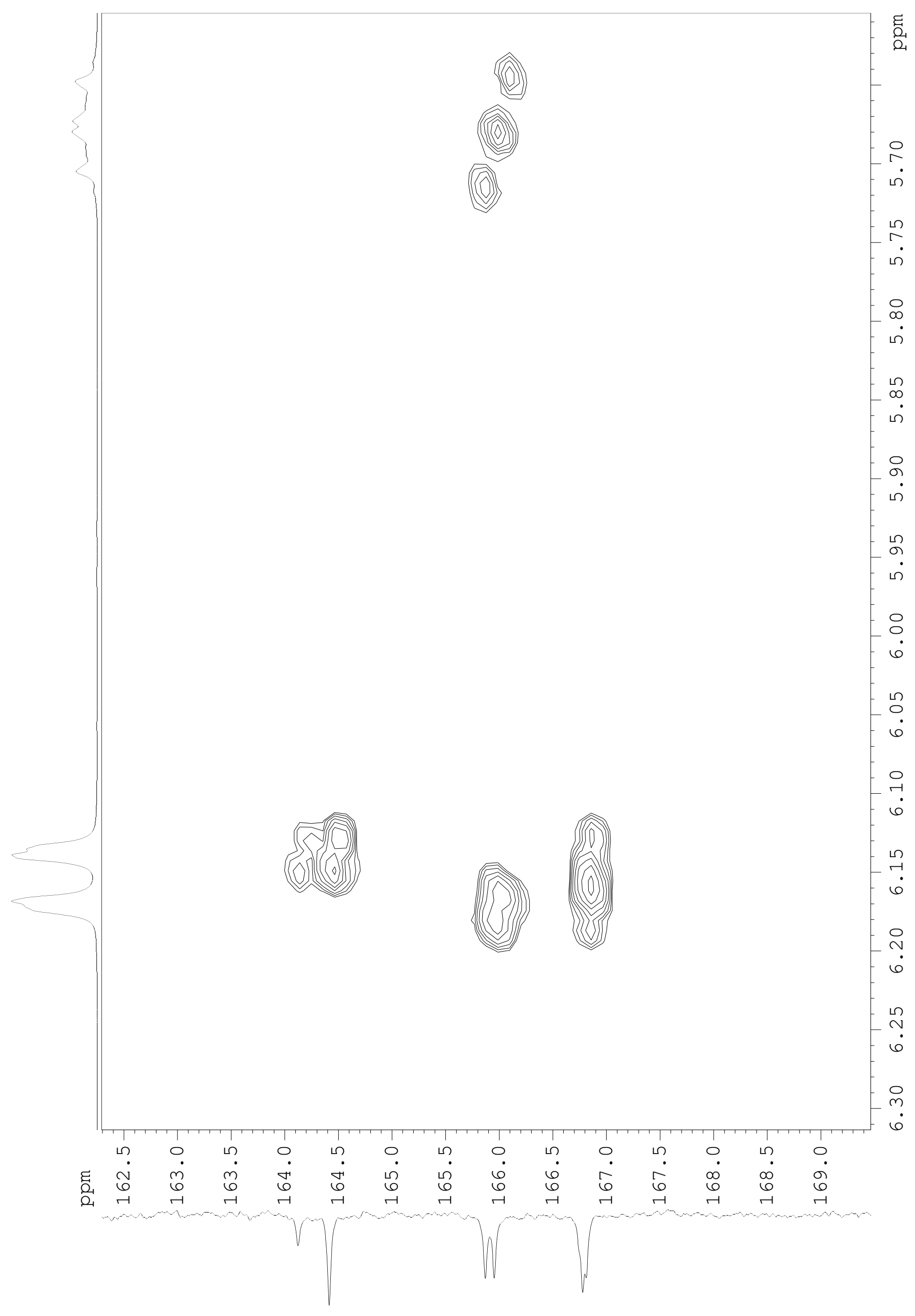




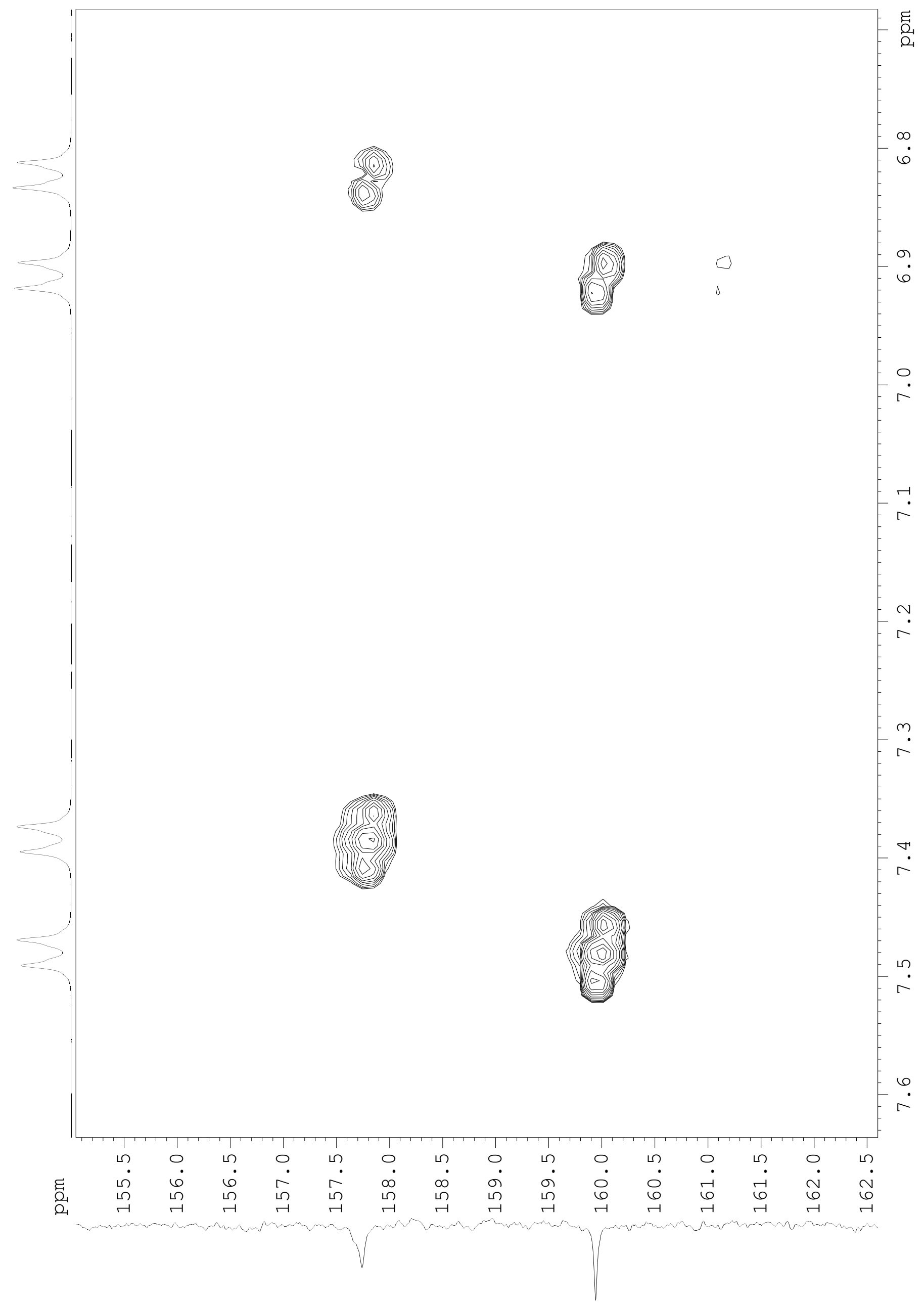




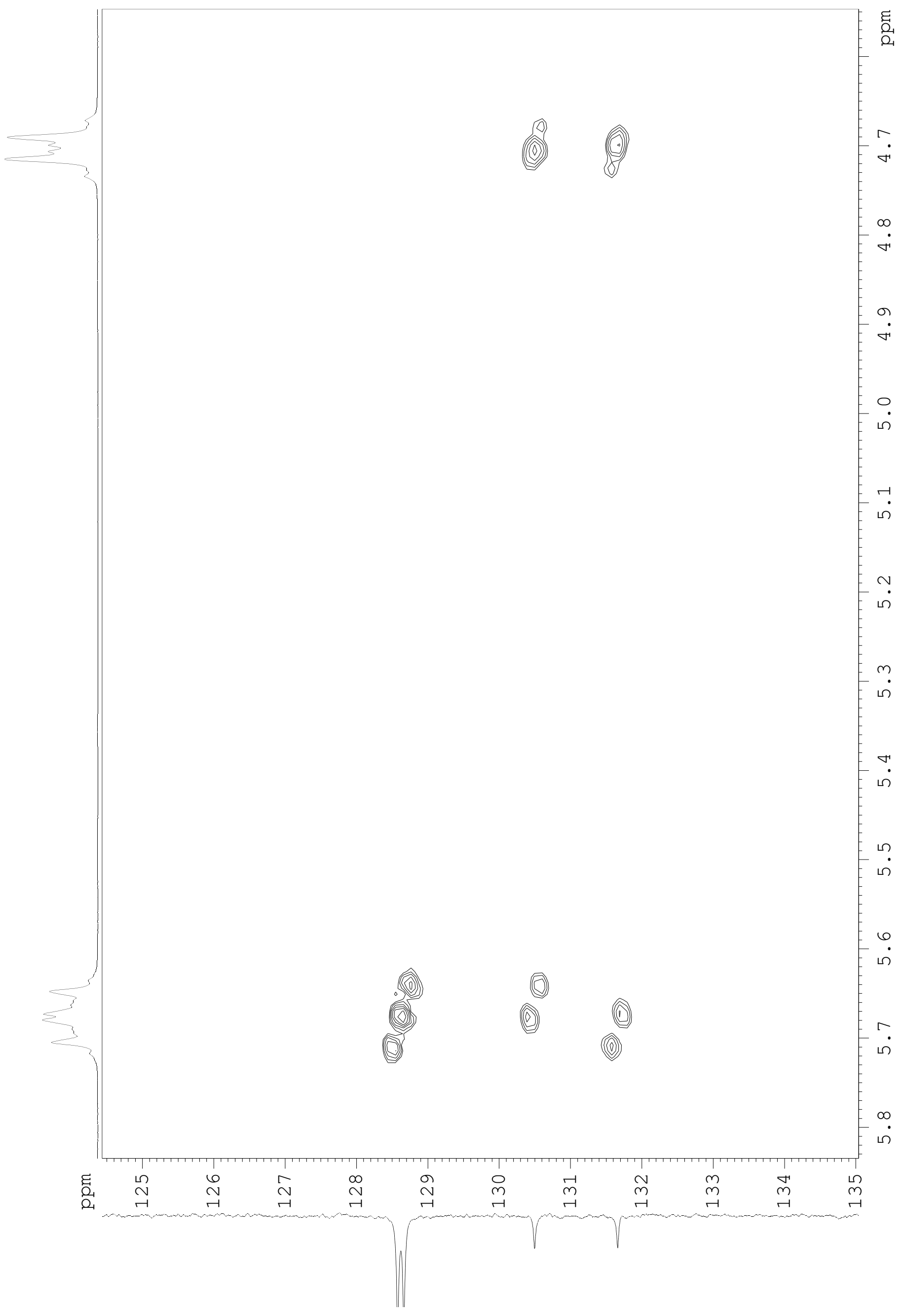




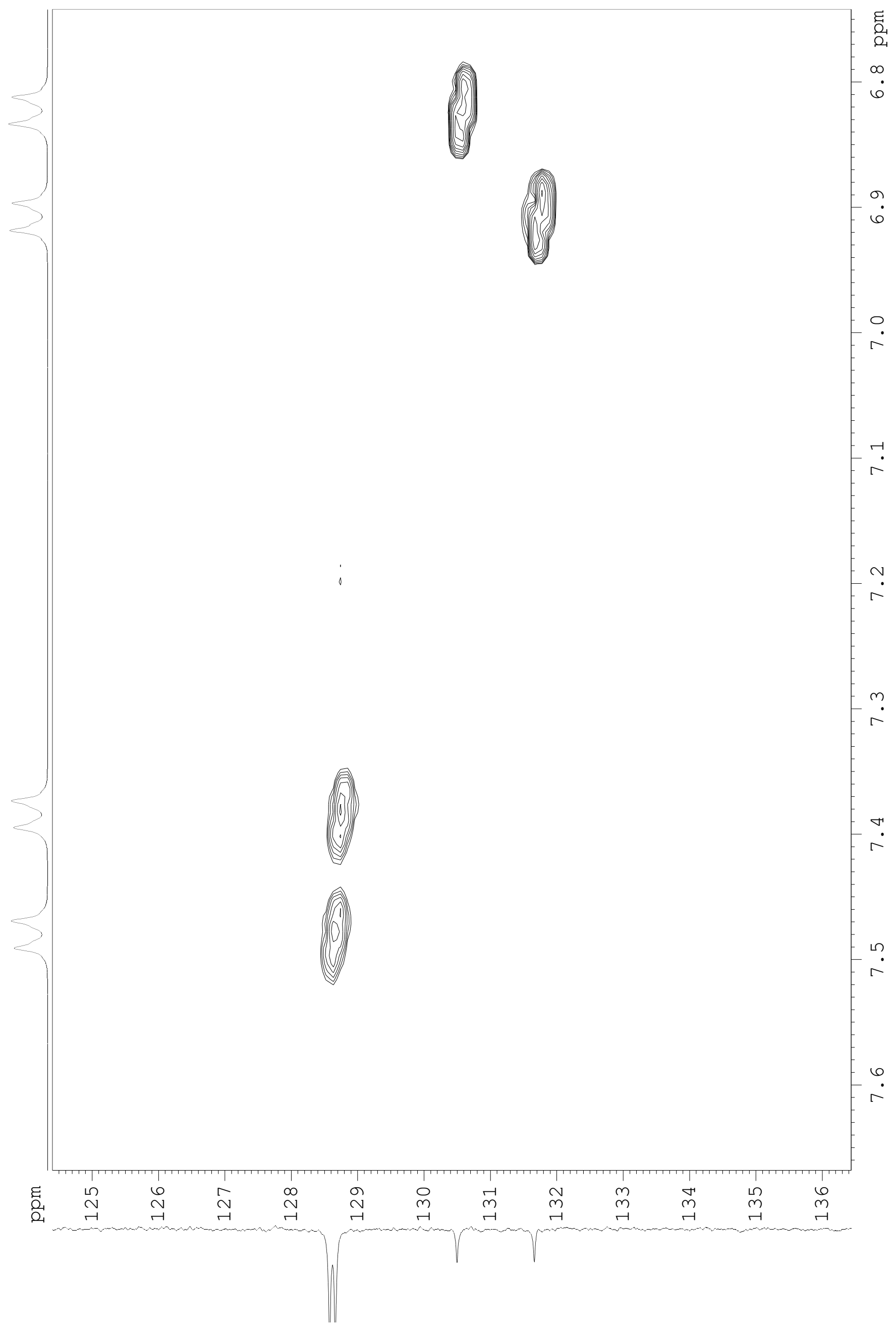




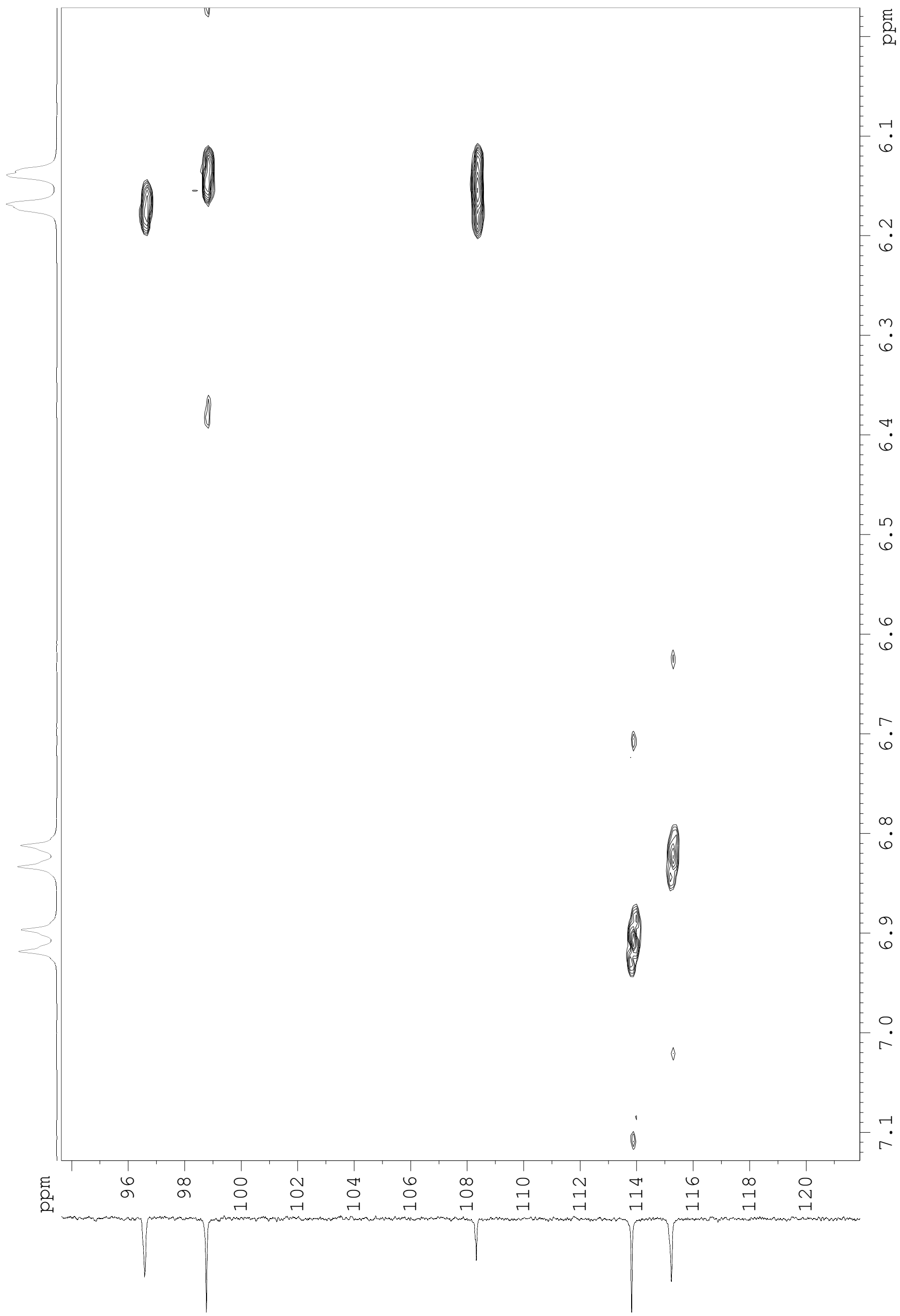




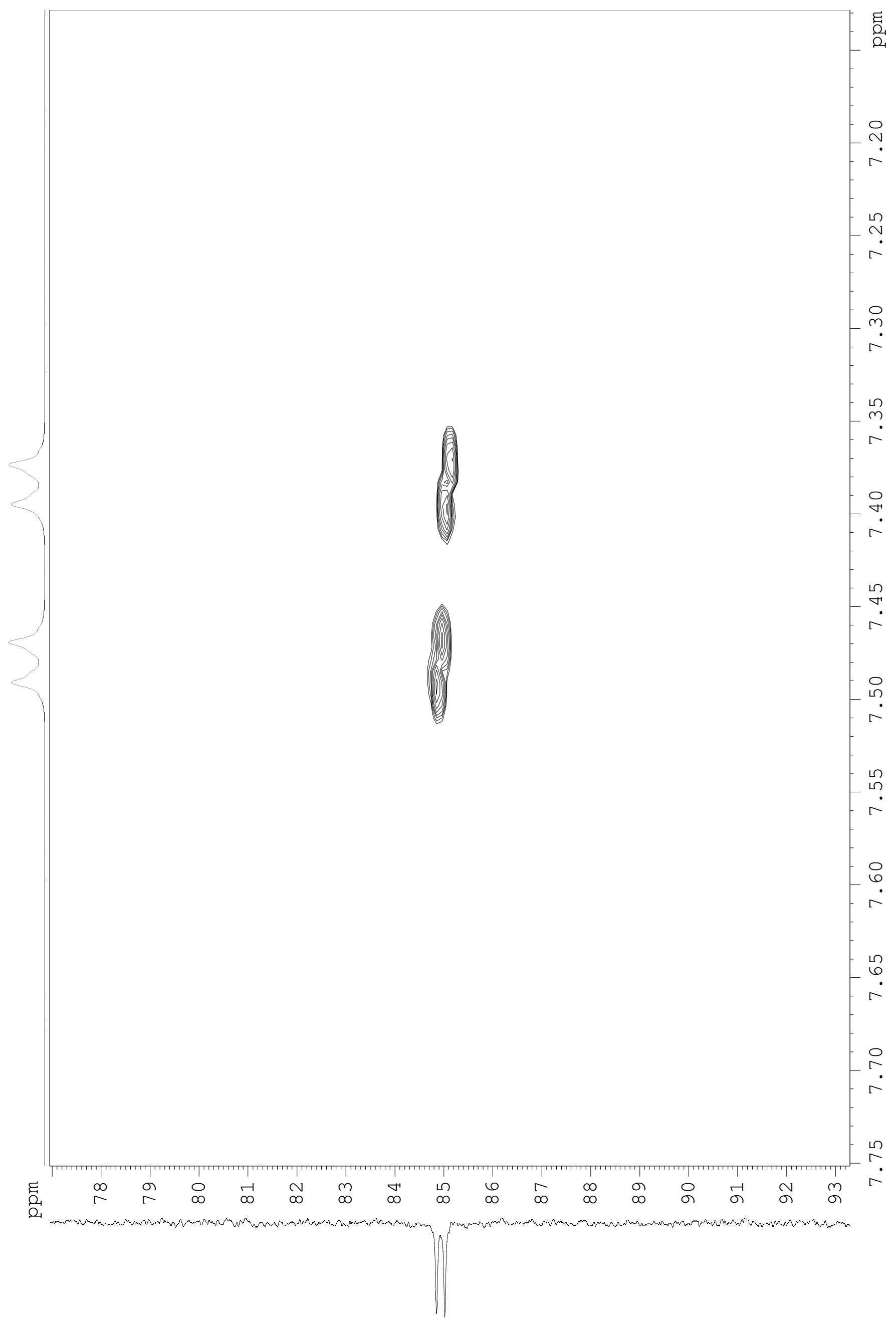




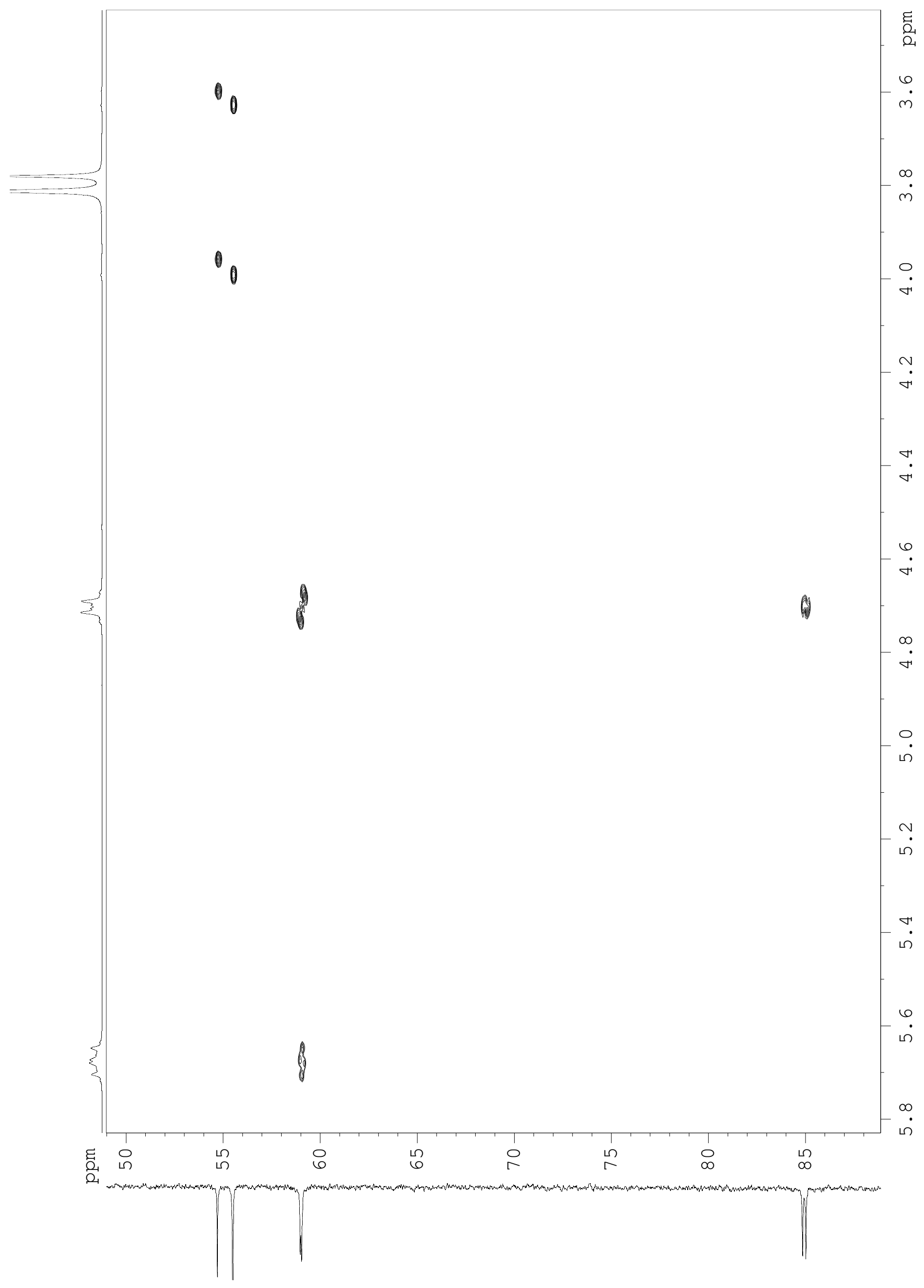




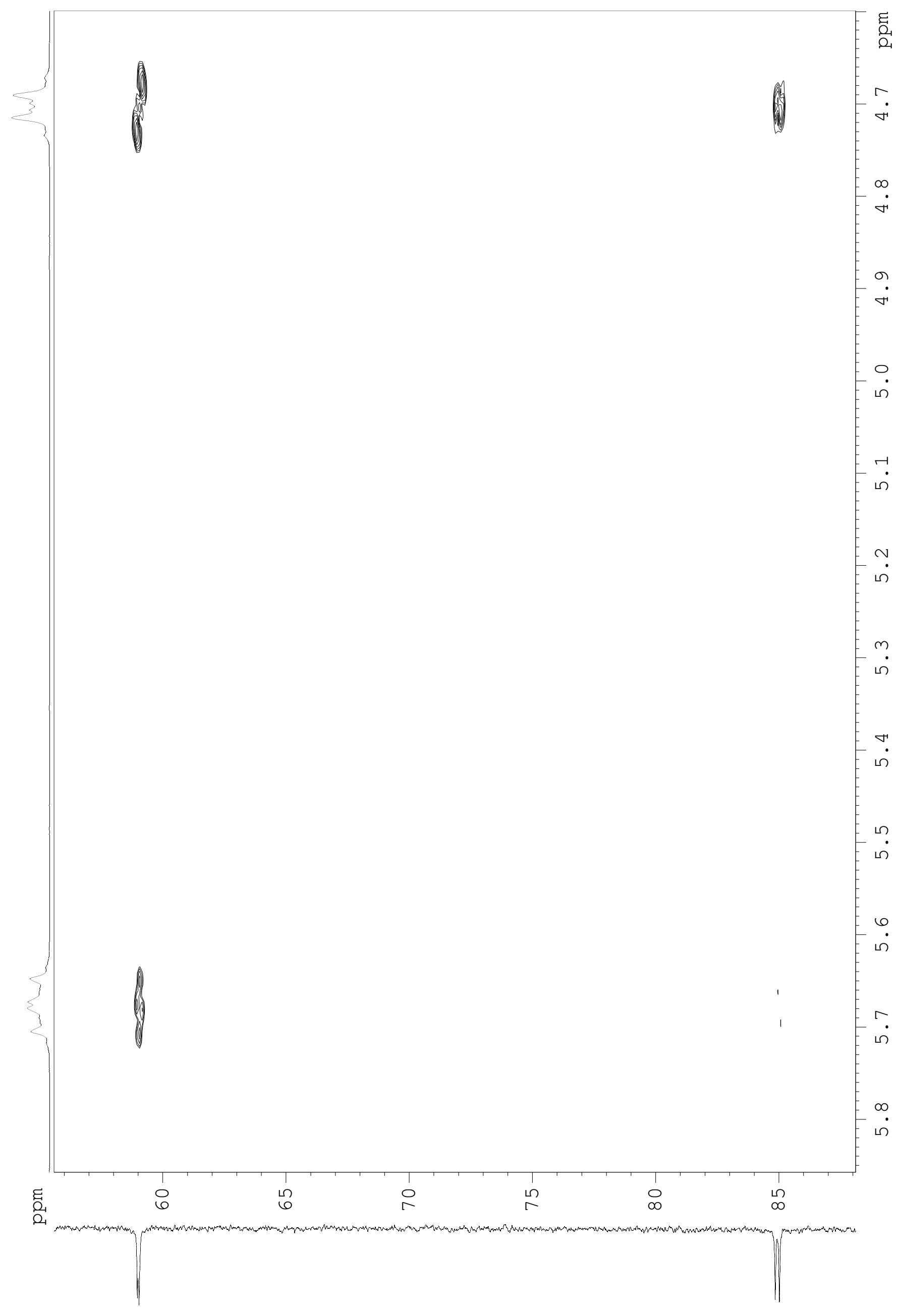


$099^{\circ} 9-$

$269^{\circ} \mathrm{s}^{-}$

S五 9

$8 \pi \tau \cdot 9=$

$6 L I \cdot 9-$

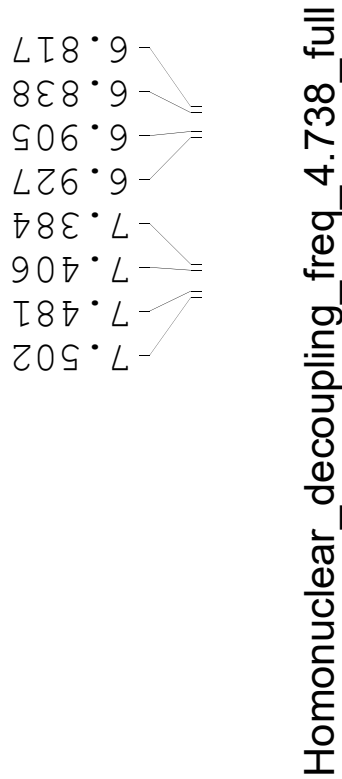

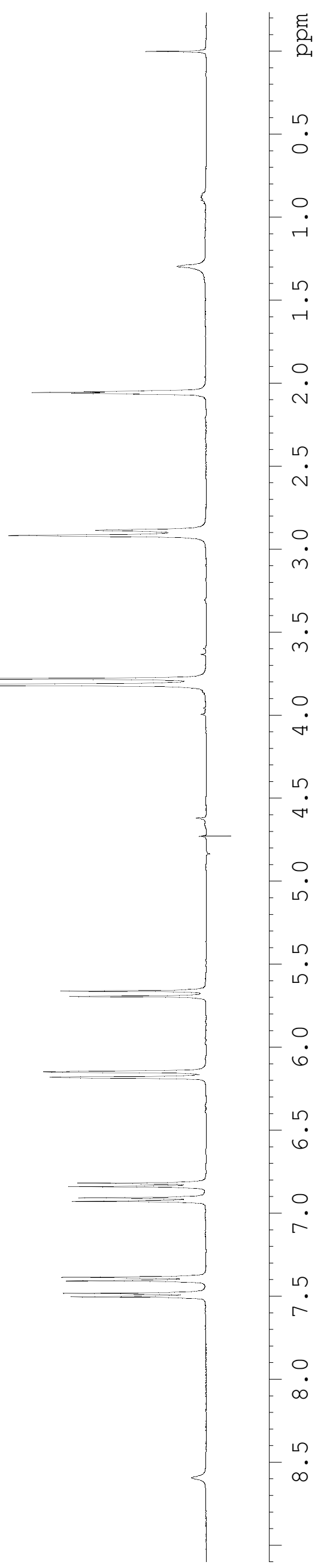


$099^{\circ} \mathrm{S}^{-}$

$269^{\circ} 9$

SธI 9

$8 \nabla \tau \cdot 9$ -

$6 L I \cdot 9-$
Lโ8 9 -

$8 \varepsilon 8 \cdot 9$ -

$906 \cdot 9$

$426 \cdot 9$ -
Ђ $8 \varepsilon^{\circ} L$

$907 \cdot L$

โ8 ${ }^{\circ} L$

ZOS $L$

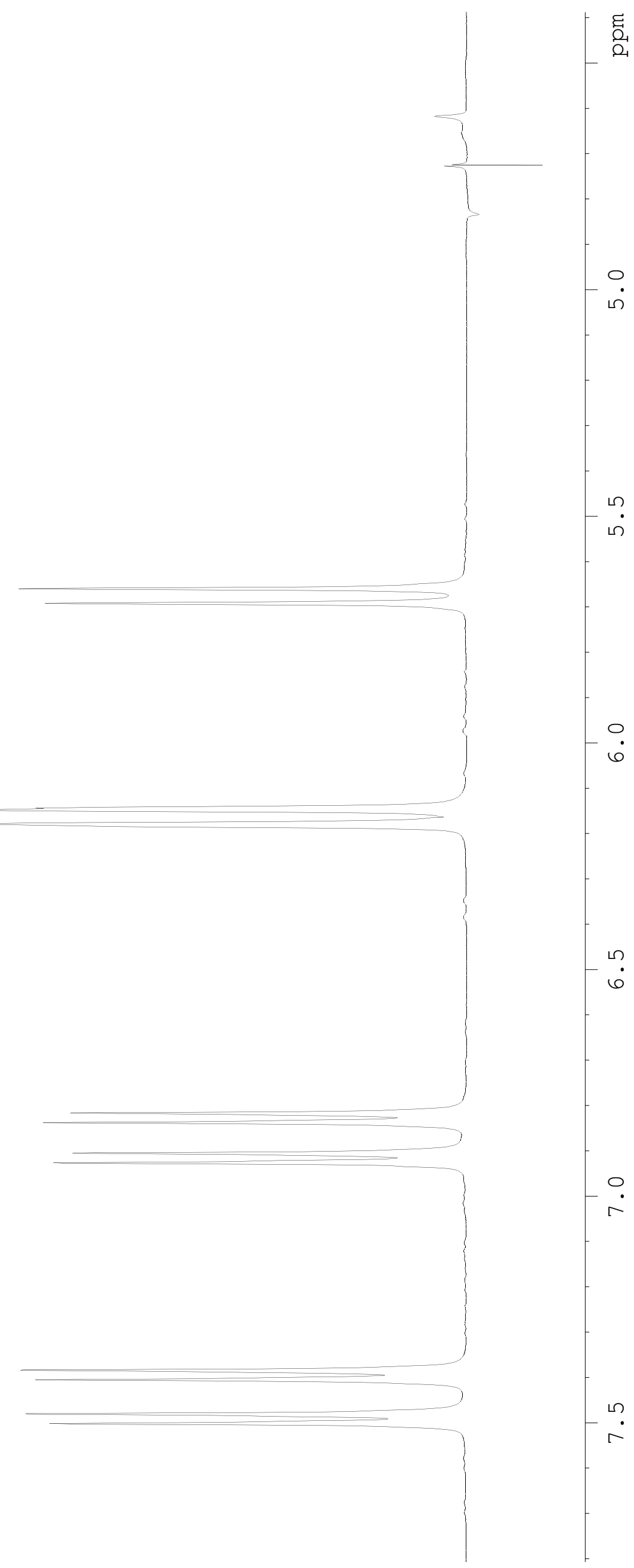




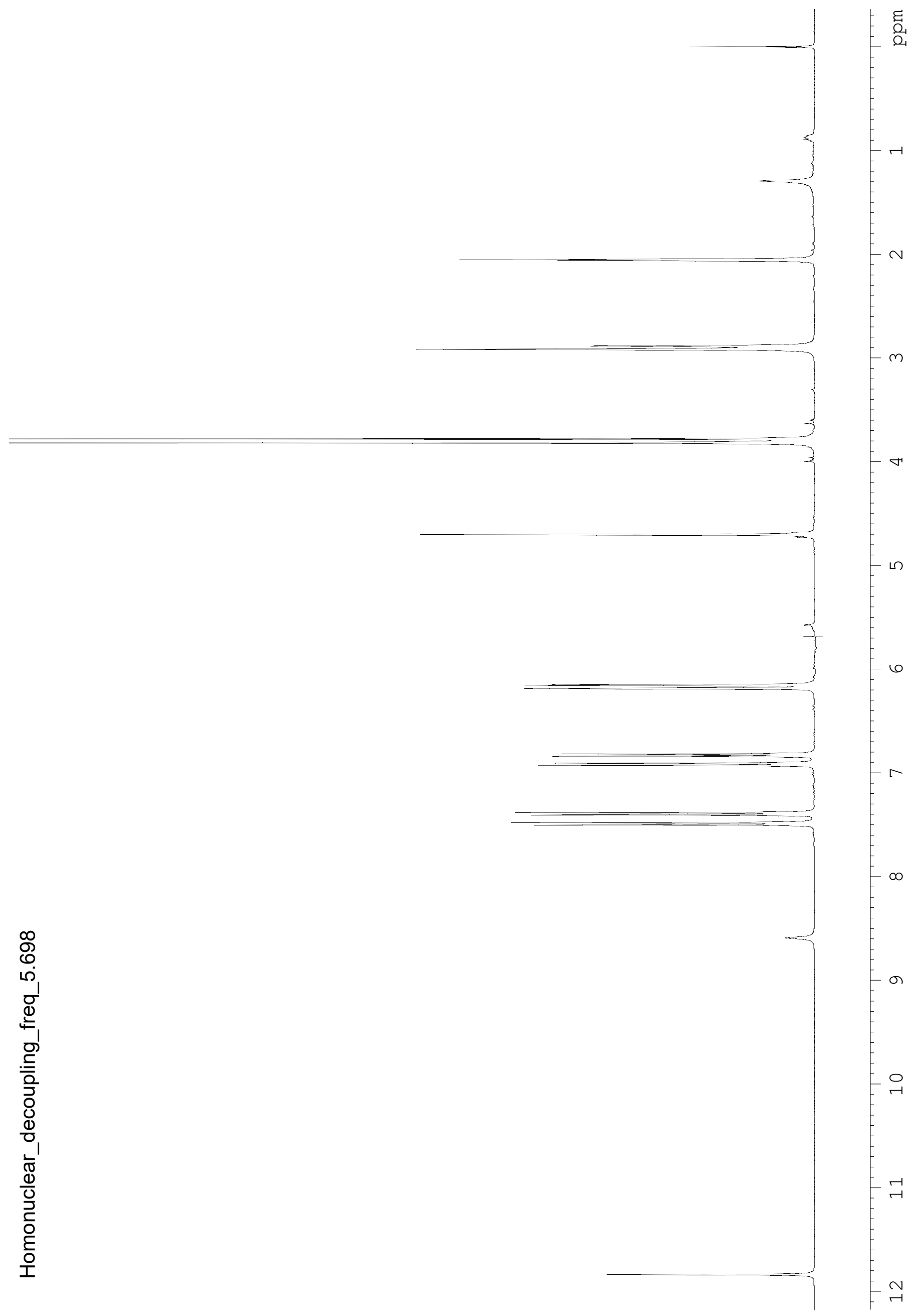


$98 \varepsilon^{\circ} L$ $90 \overline{7}^{\circ} L$

โ8 $0^{\circ} L$ EOS $L$ 


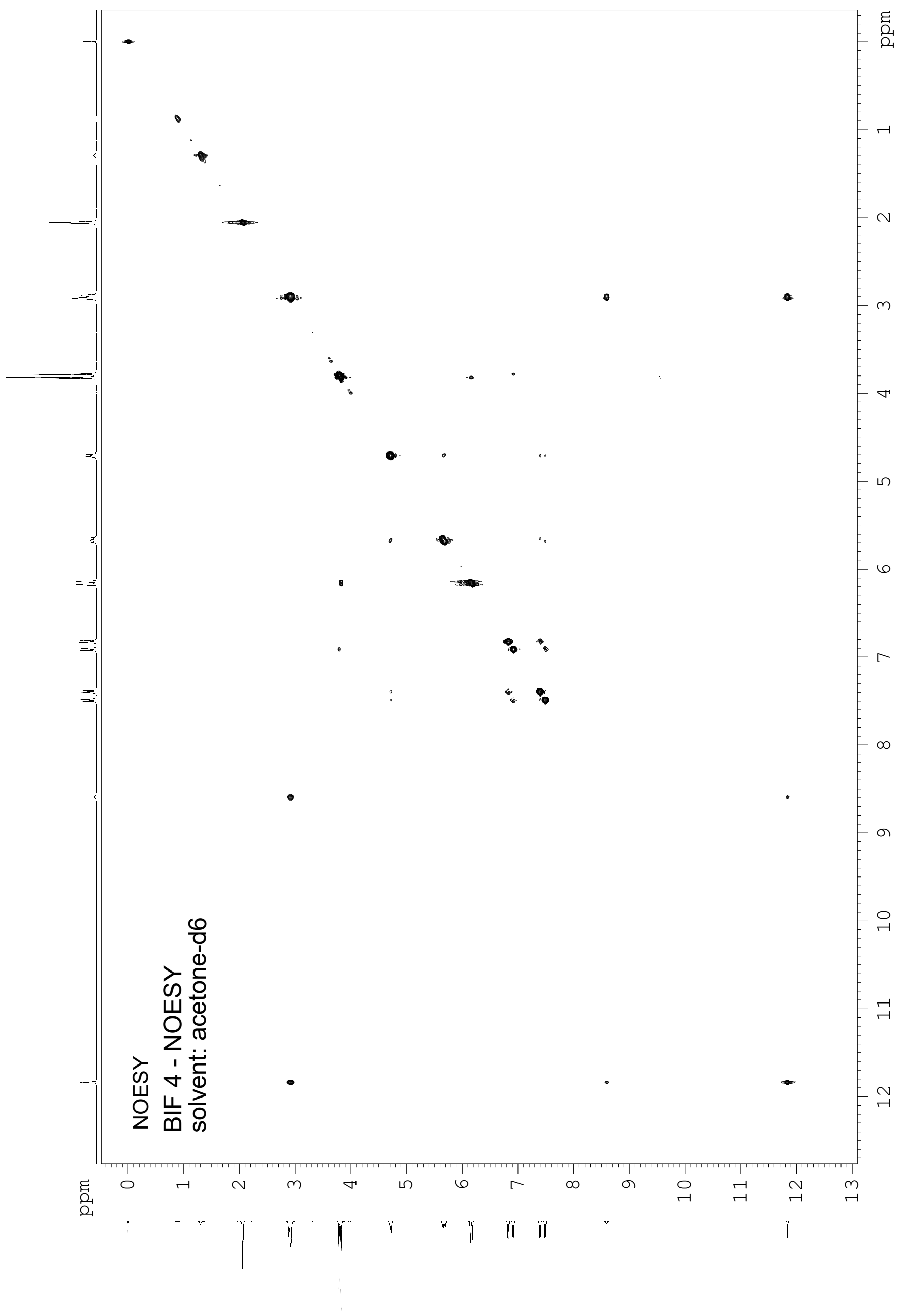




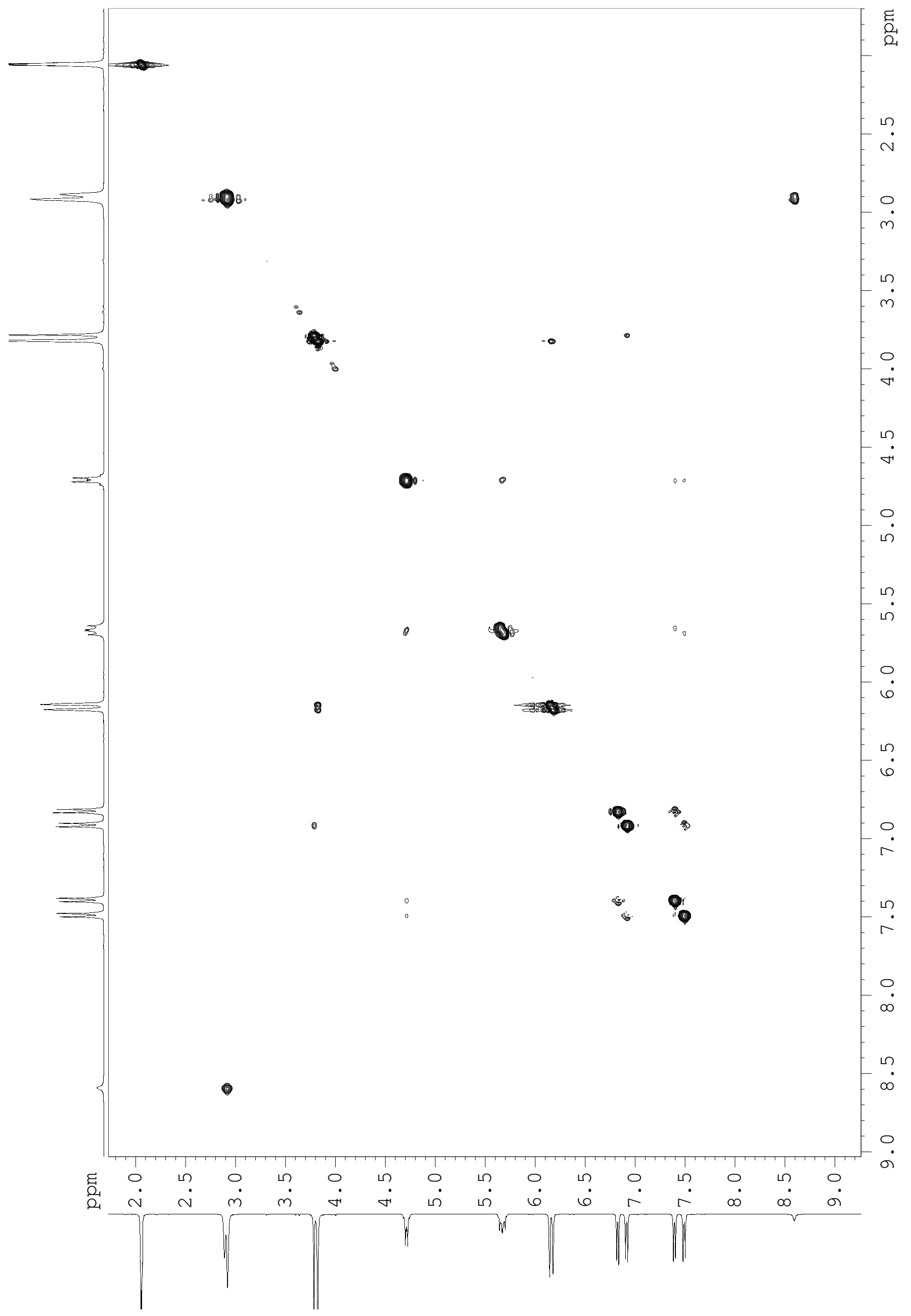




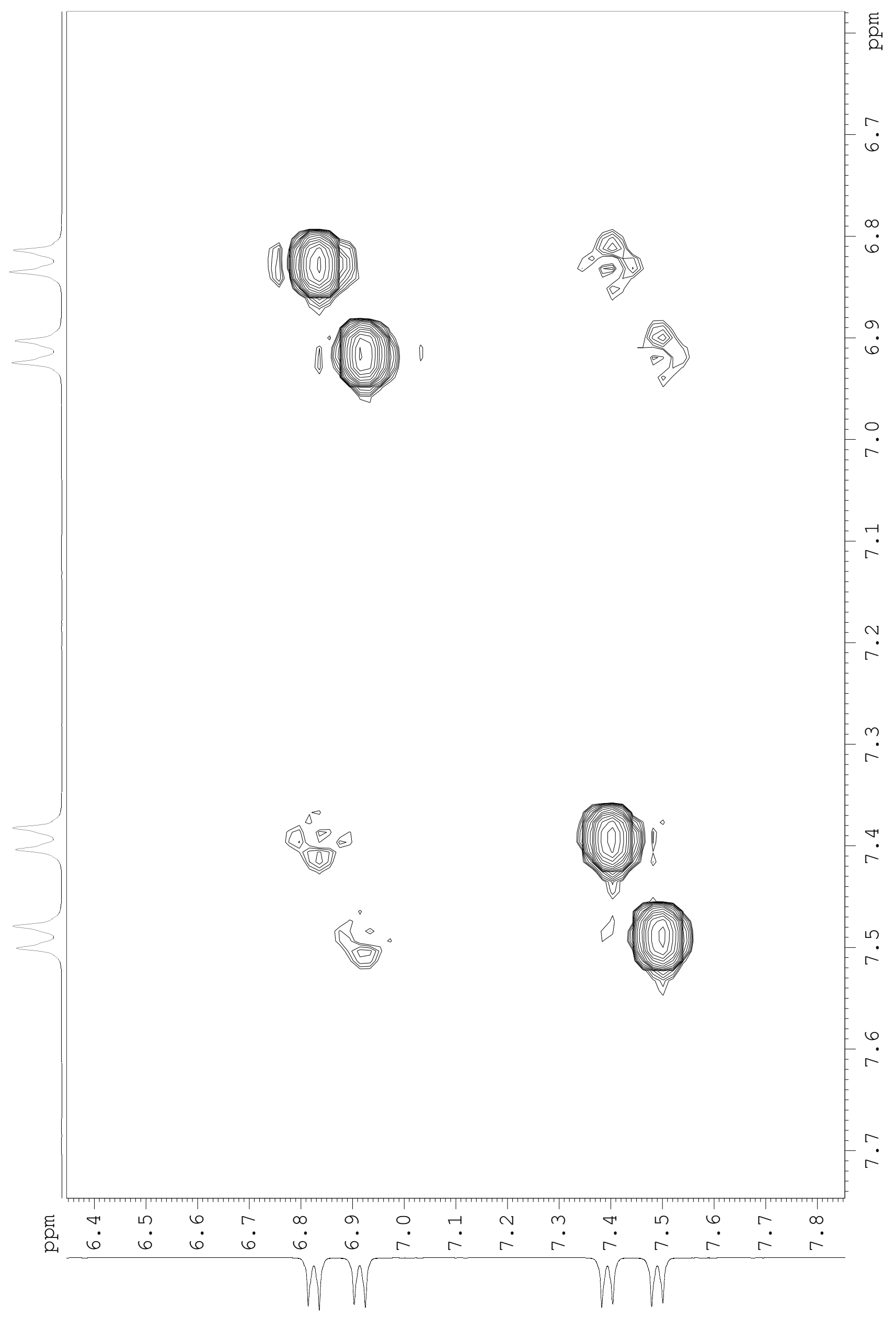




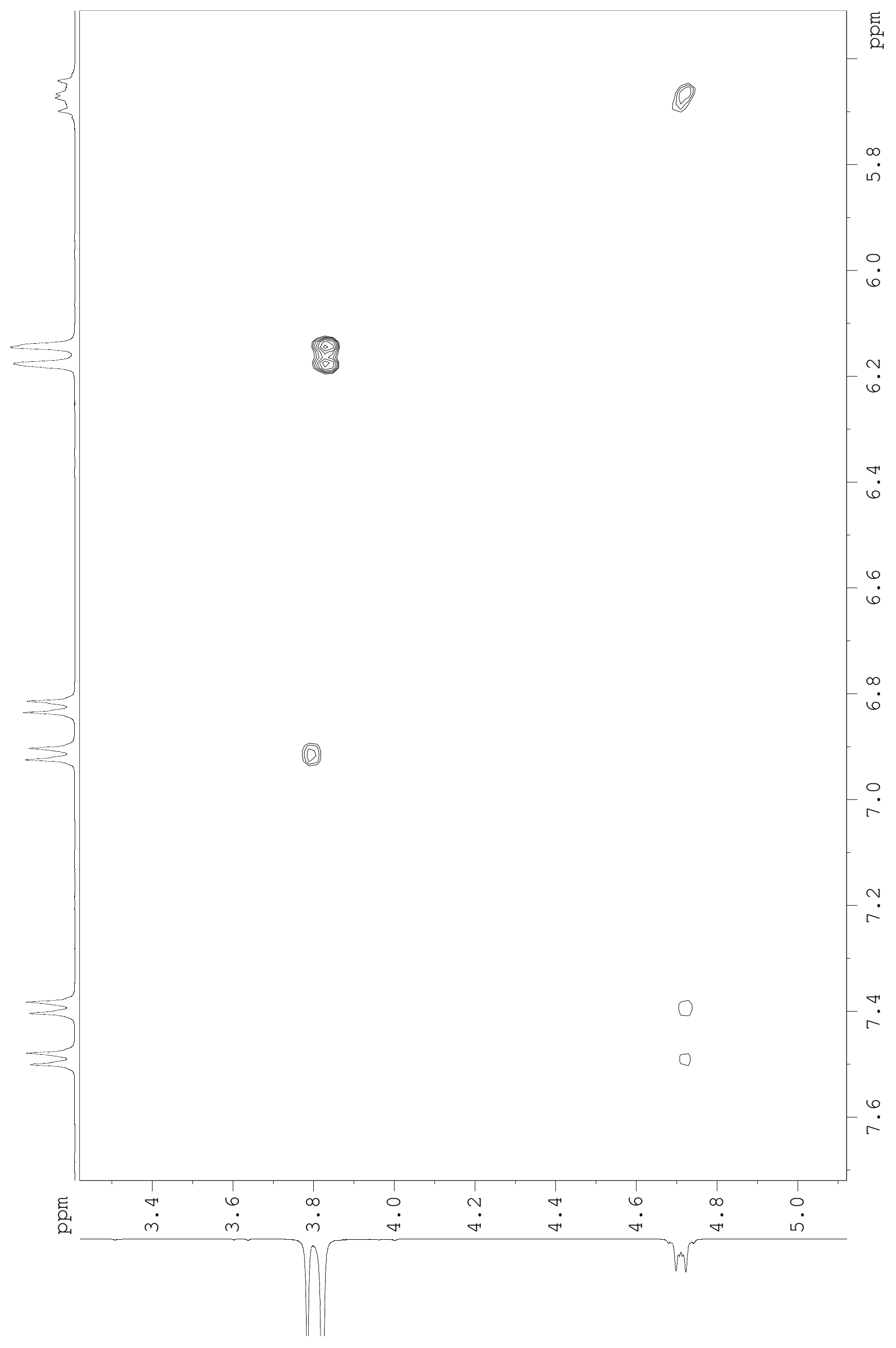




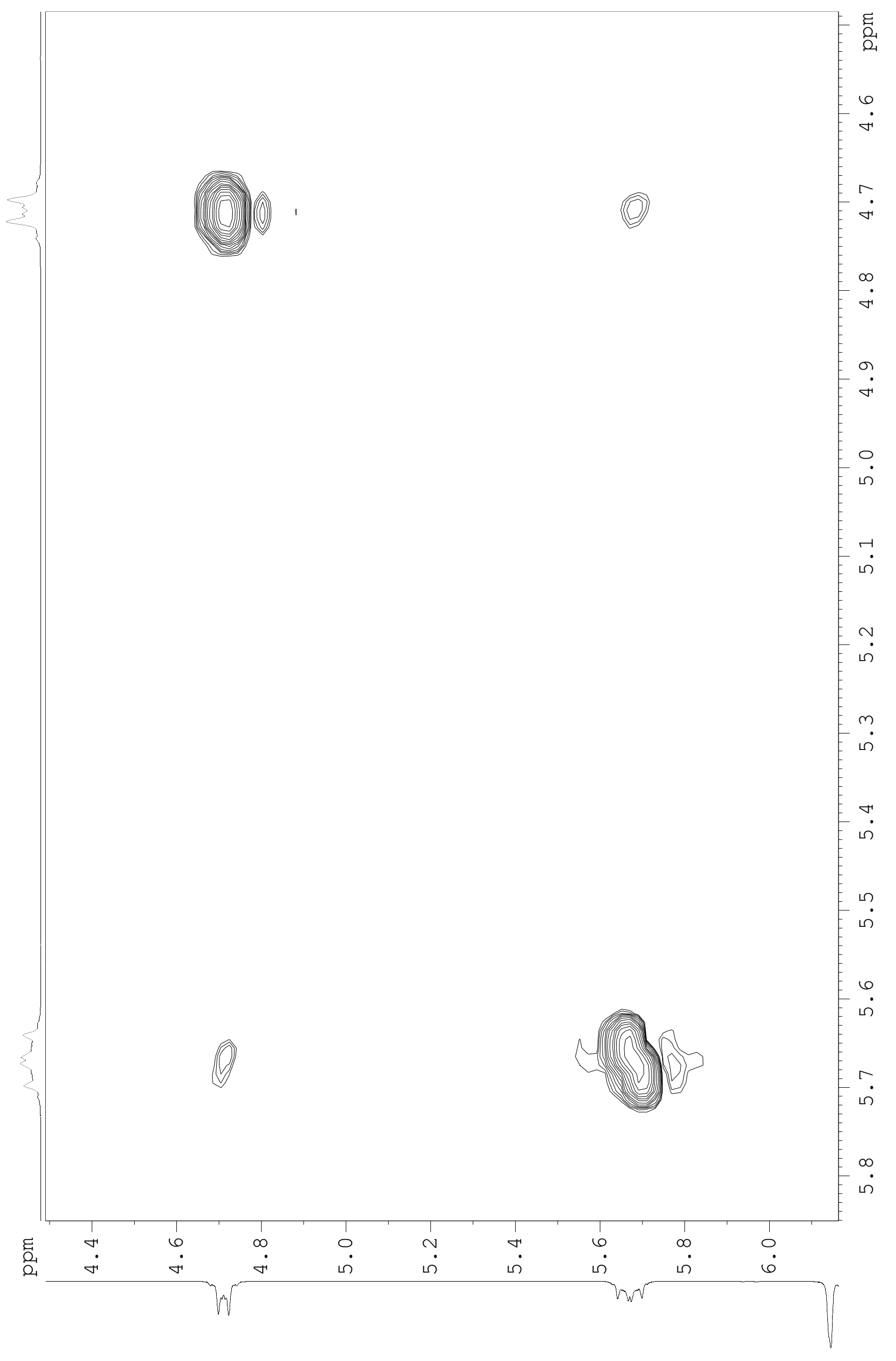




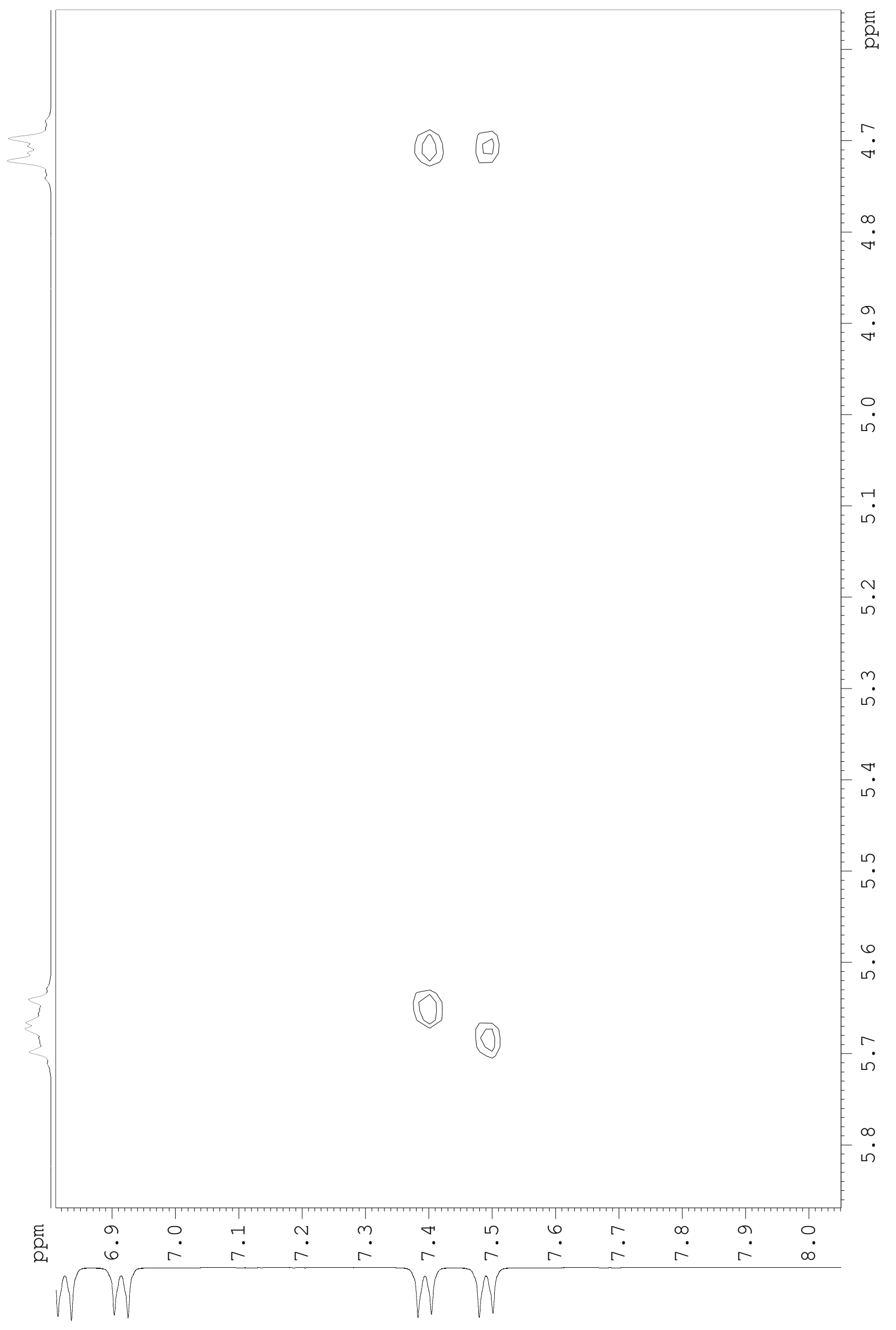




$$
\frac{1}{i}
$$




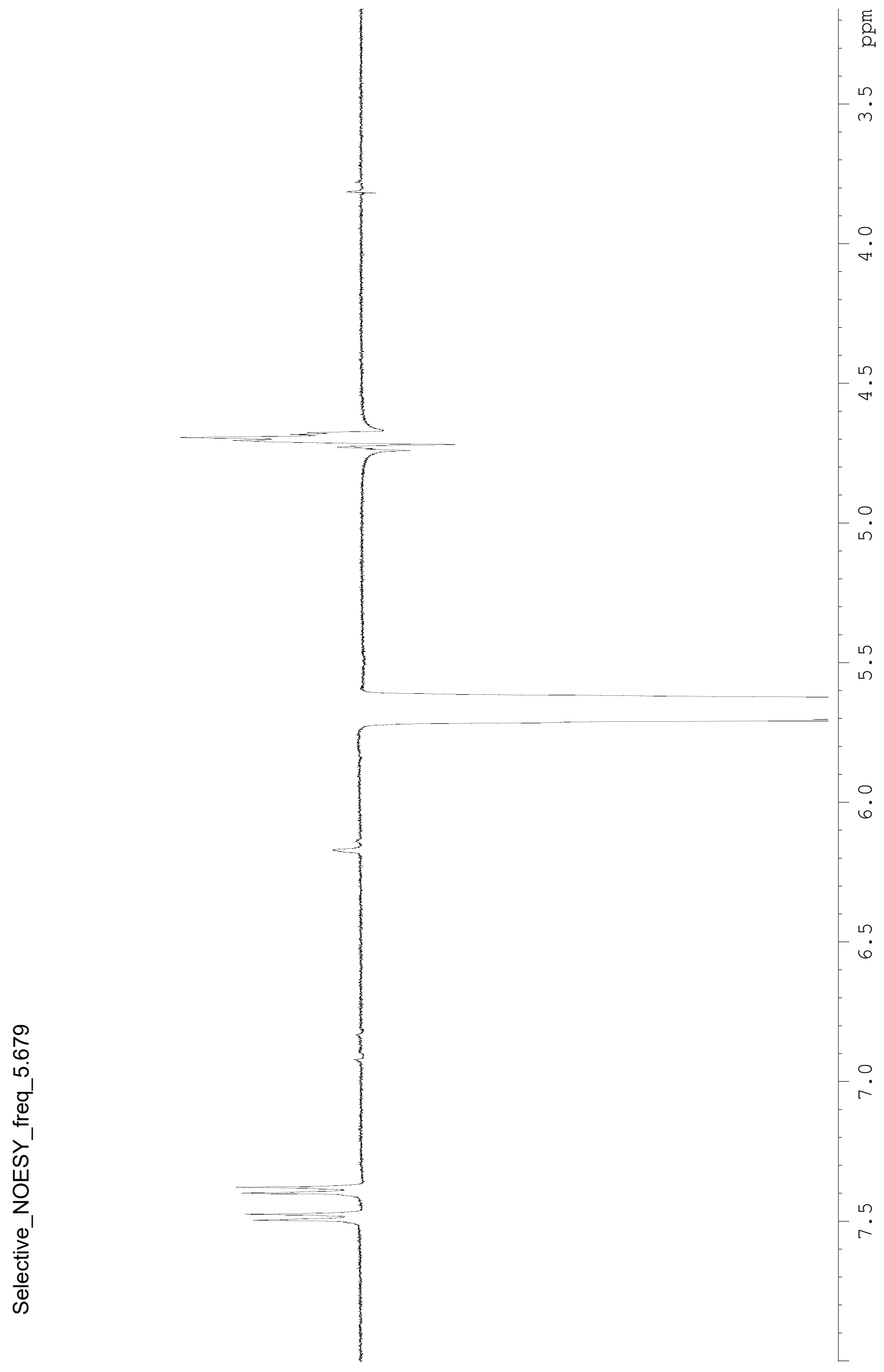




\section{Segunda injeção}

\section{Ouratein A}

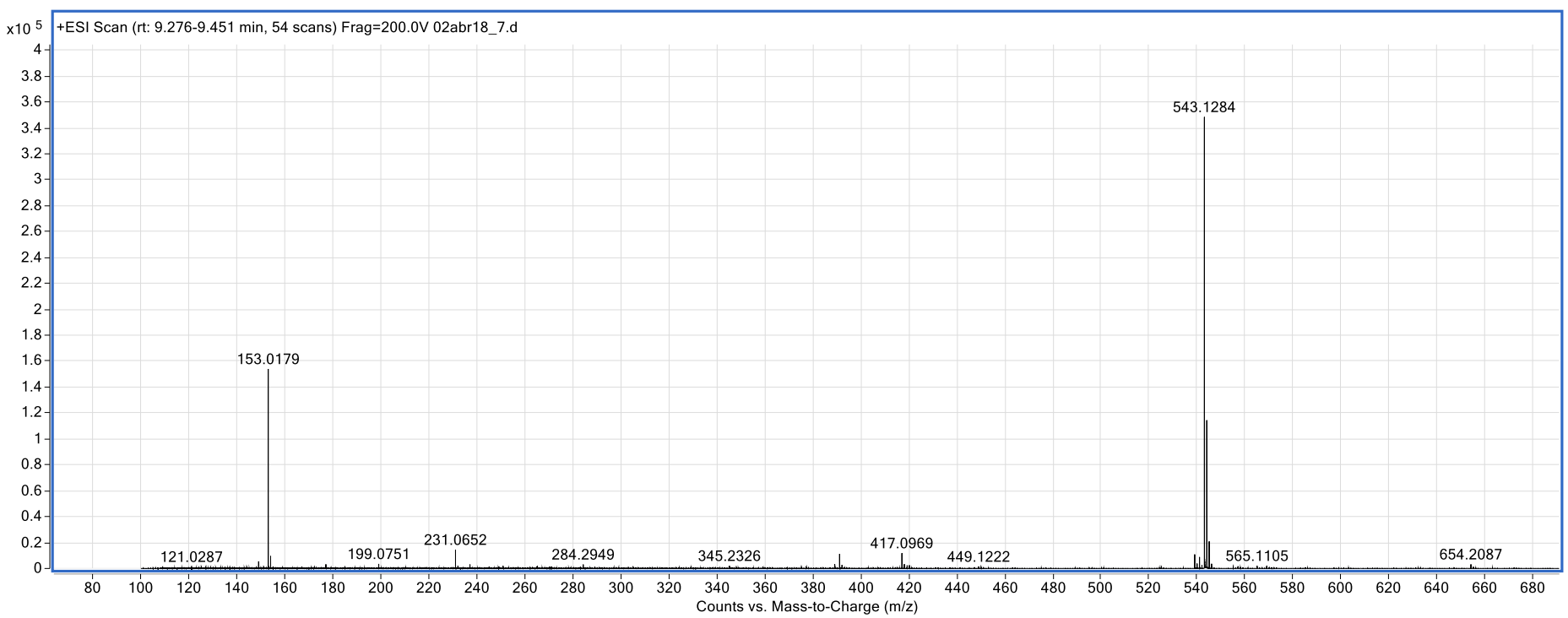

\section{Ouratein B}

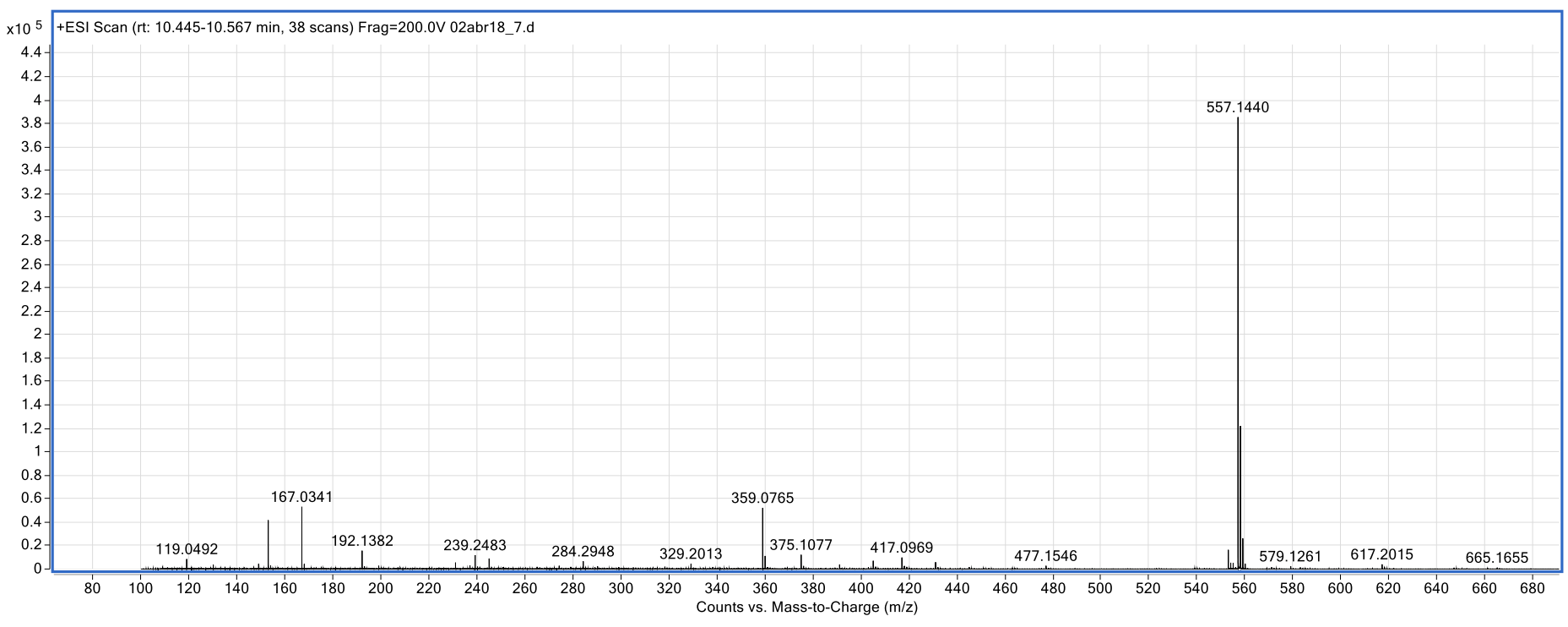




\section{Ouratein C}

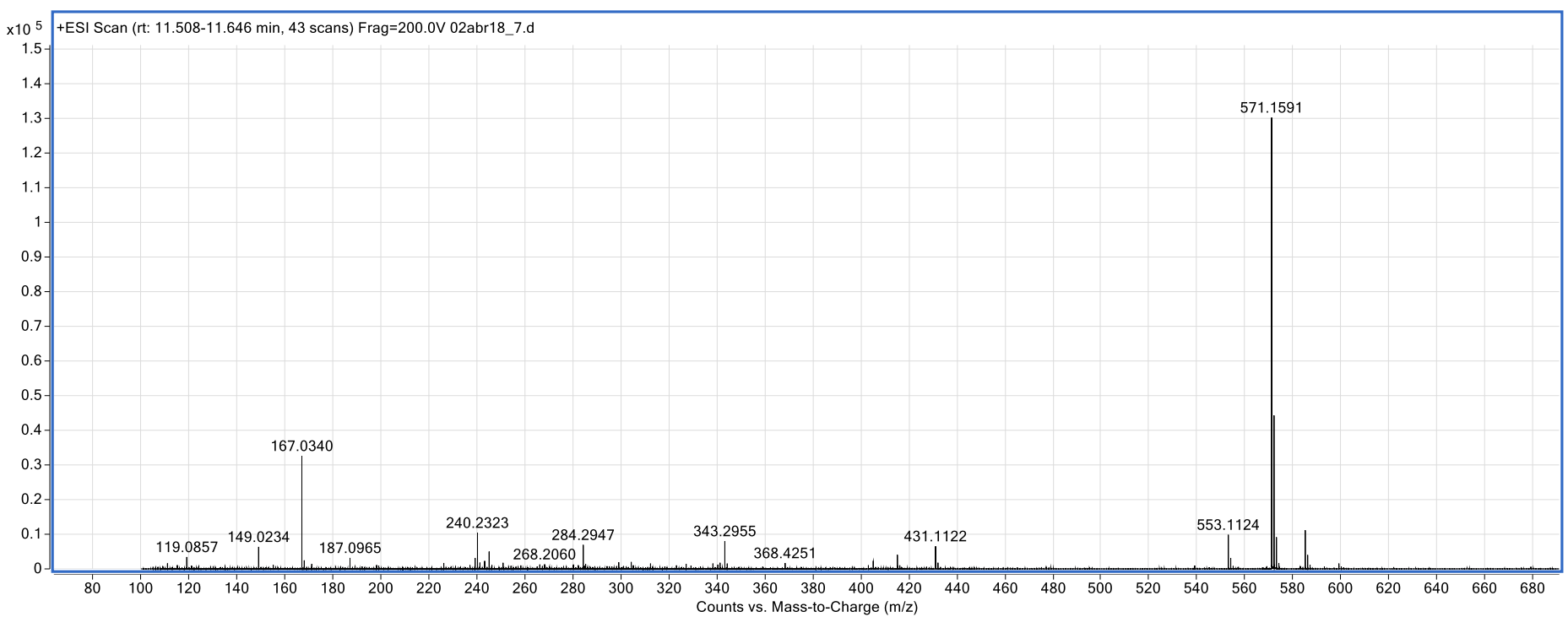

\section{Ouratein D}

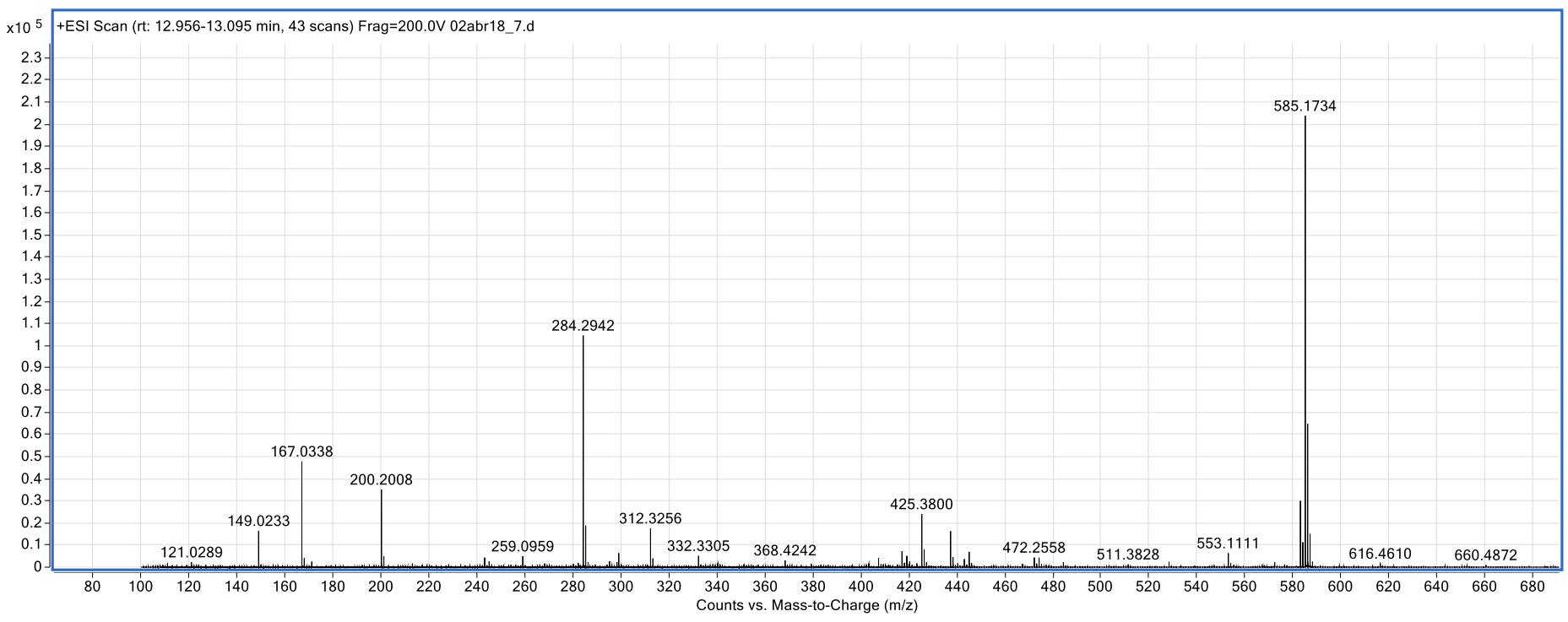




\section{Glico B2}
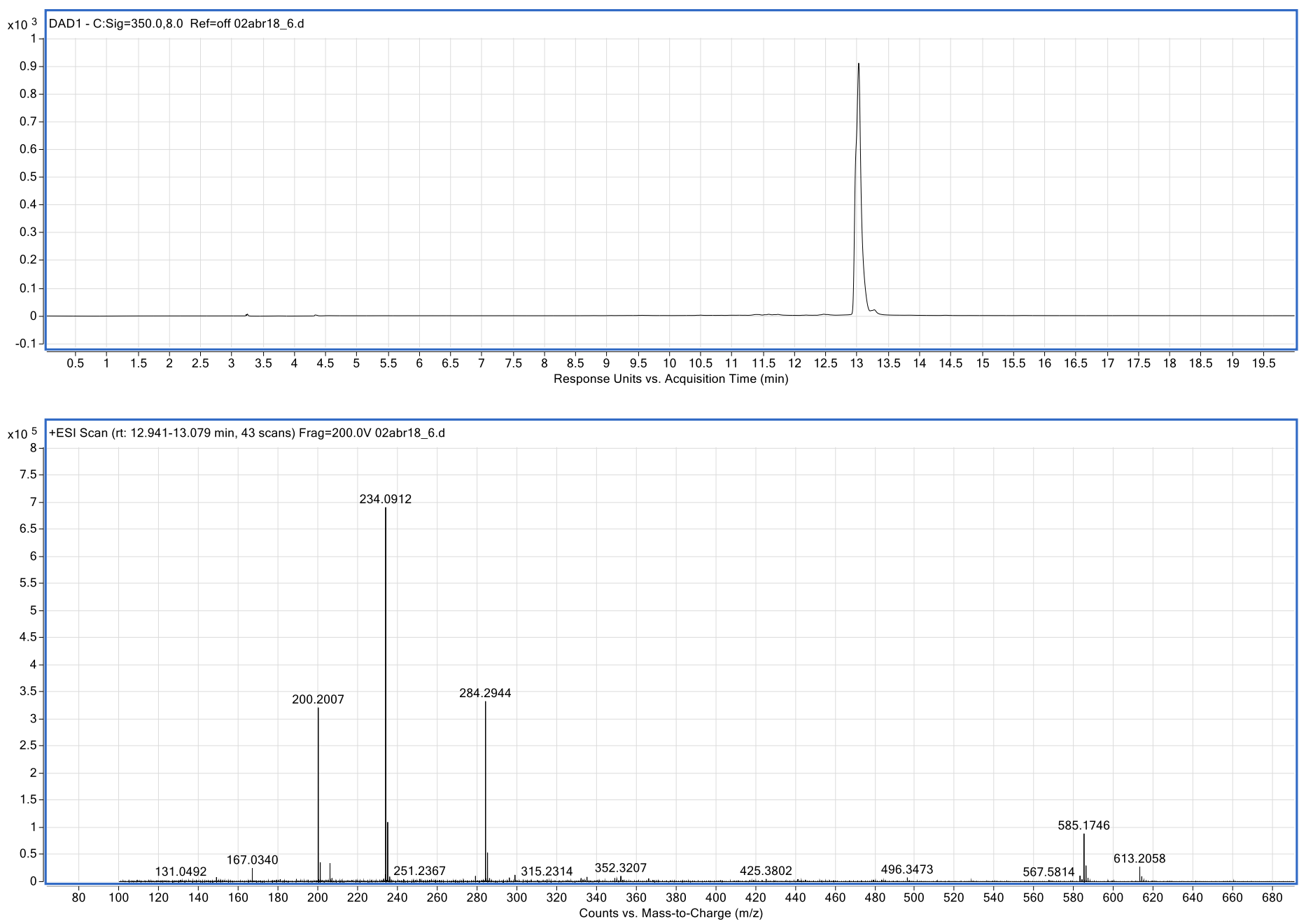